Supporting Information

\title{
Synthesis and Conformational Analysis of Parent Perhydroazulenes Reveals an Energetically Preferred cis Ring Fusion
}

\author{
Fumito Saito, ${ }^{1}$ Jonathan Becker, ${ }^{2}$ and Peter R. Schreiner ${ }^{1 *}$ \\ ${ }^{1}$ Institute of Organic Chemistry, Justus Liebig University \\ Heinrich-Buff-Ring 17, 35392 Giessen, Germany \\ ${ }^{2}$ Institute of Inorganic and Analytical Chemistry, Justus Liebig University \\ Heinrich-Buff-Ring 17, 35392 Giessen, Germany \\ prs@uni-giessen.de
}

\section{Table of Contents}

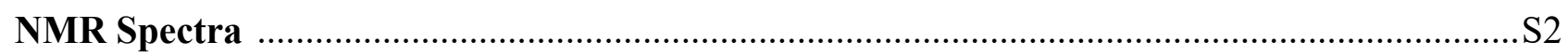

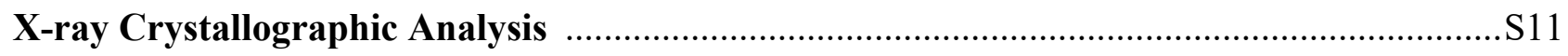

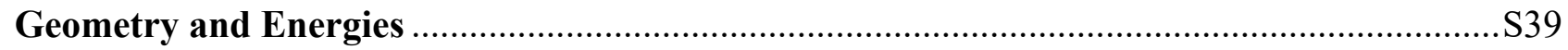

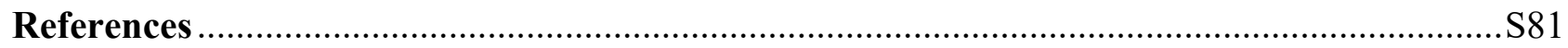




\section{NMR Spectra}

1,3-Dithiolane 5

${ }^{1} \mathrm{H}$ NMR (400 MHz, $\left.\mathrm{CDCl}_{3}\right)$<smiles>C1CCC23CCSC[SH]2C(C1)C3</smiles>

๑ஸ்.

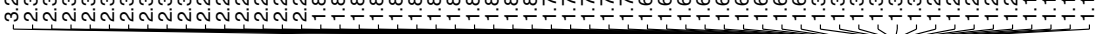

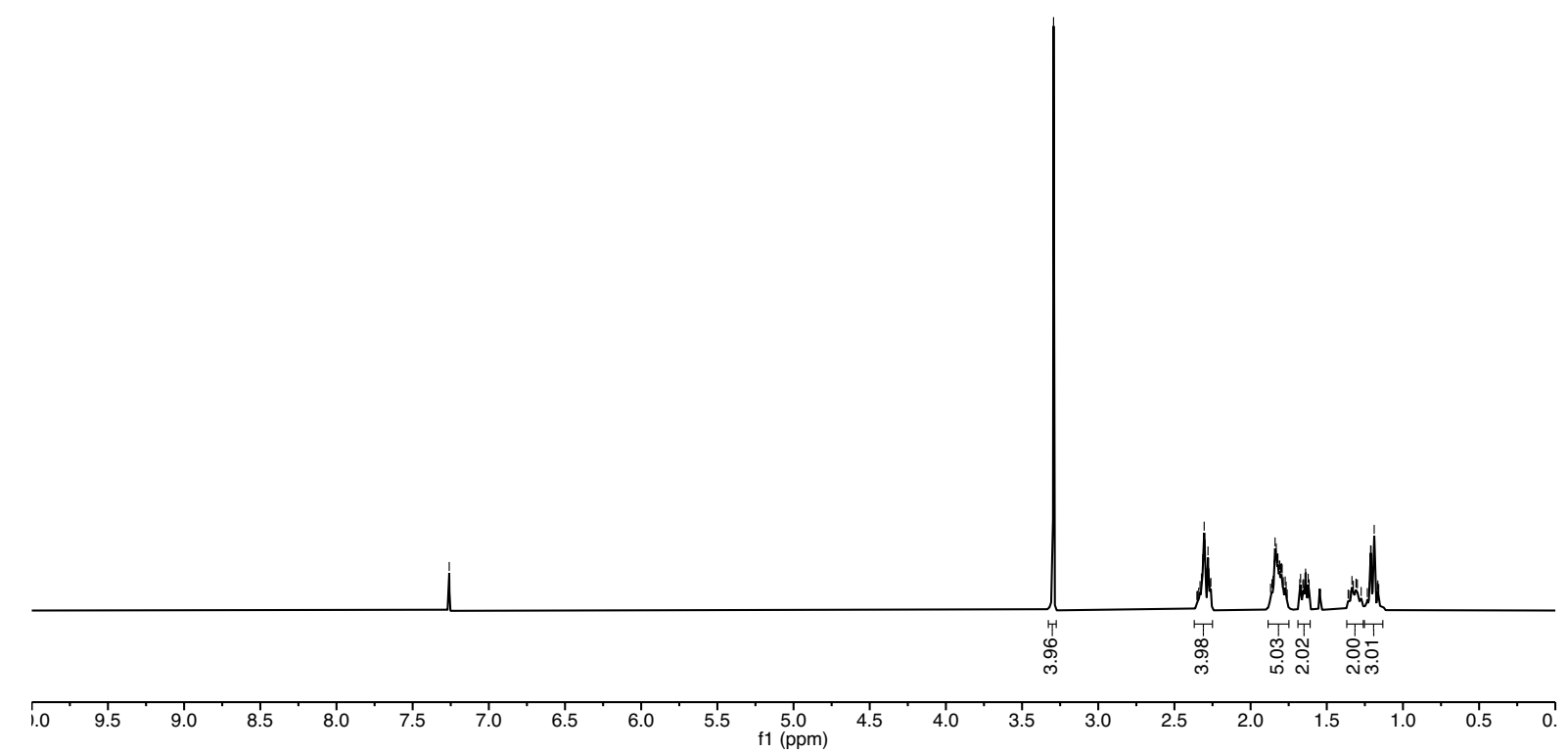

${ }^{13} \mathrm{C}$ NMR (101 MHz, $\left.\mathrm{CDCl}_{3}\right)$<smiles>C1CCC2CC3(CCCS3)CC2C1</smiles>

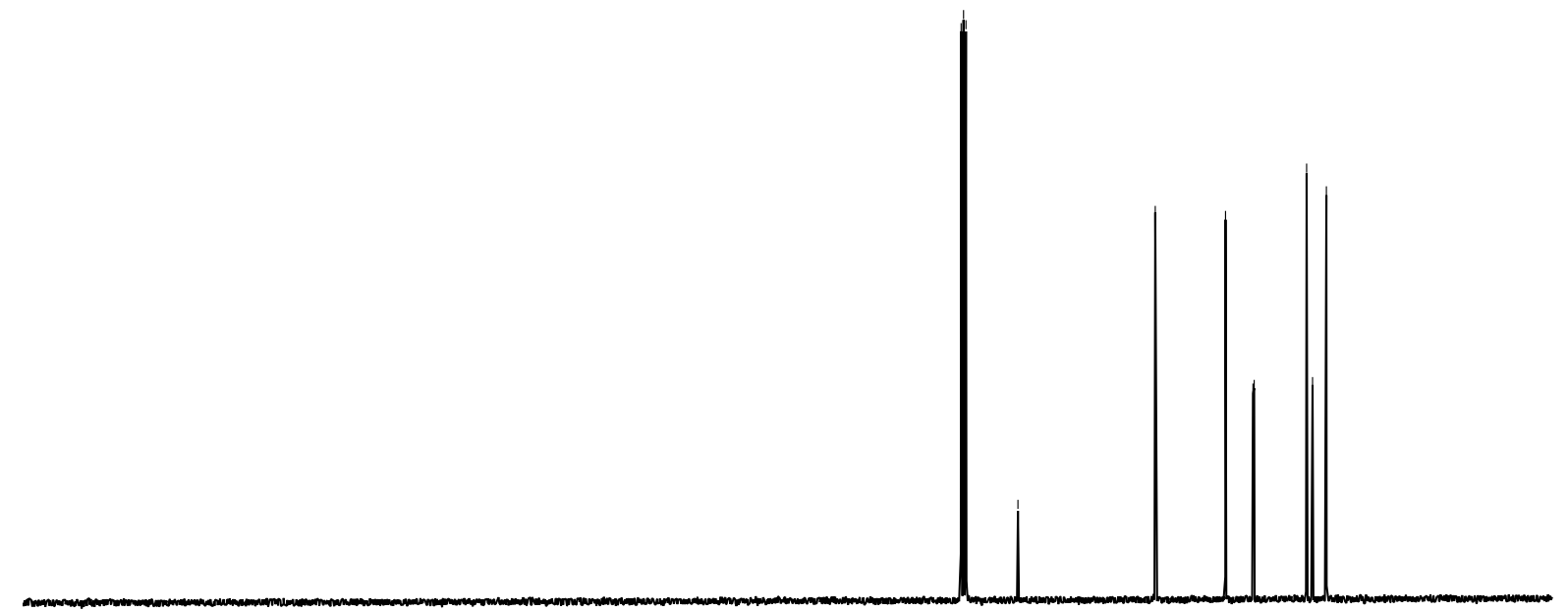

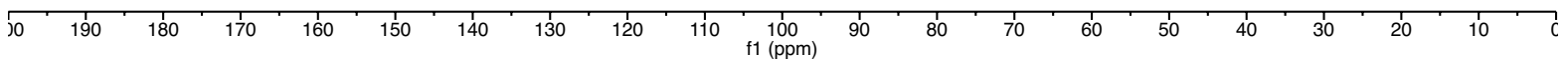


Saturated ketone 2c

${ }^{1} \mathrm{H}$ NMR (400 MHz, $\mathrm{CDCl}_{3}$ )
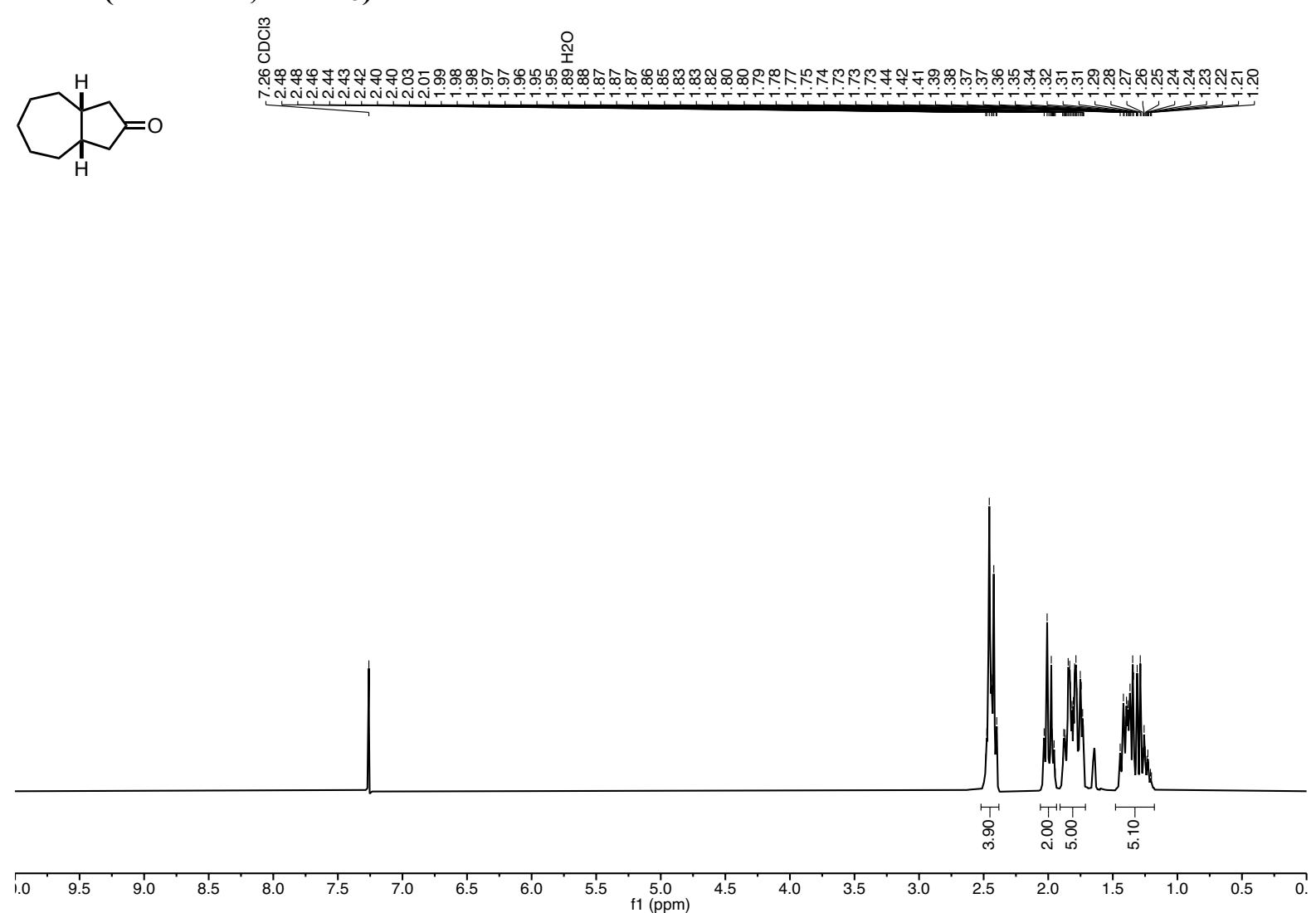

${ }^{13} \mathrm{C}$ NMR (101 MHz, $\left.\mathrm{CDCl}_{3}\right)$<smiles>O=C1CCC2CCCCC2C1</smiles>
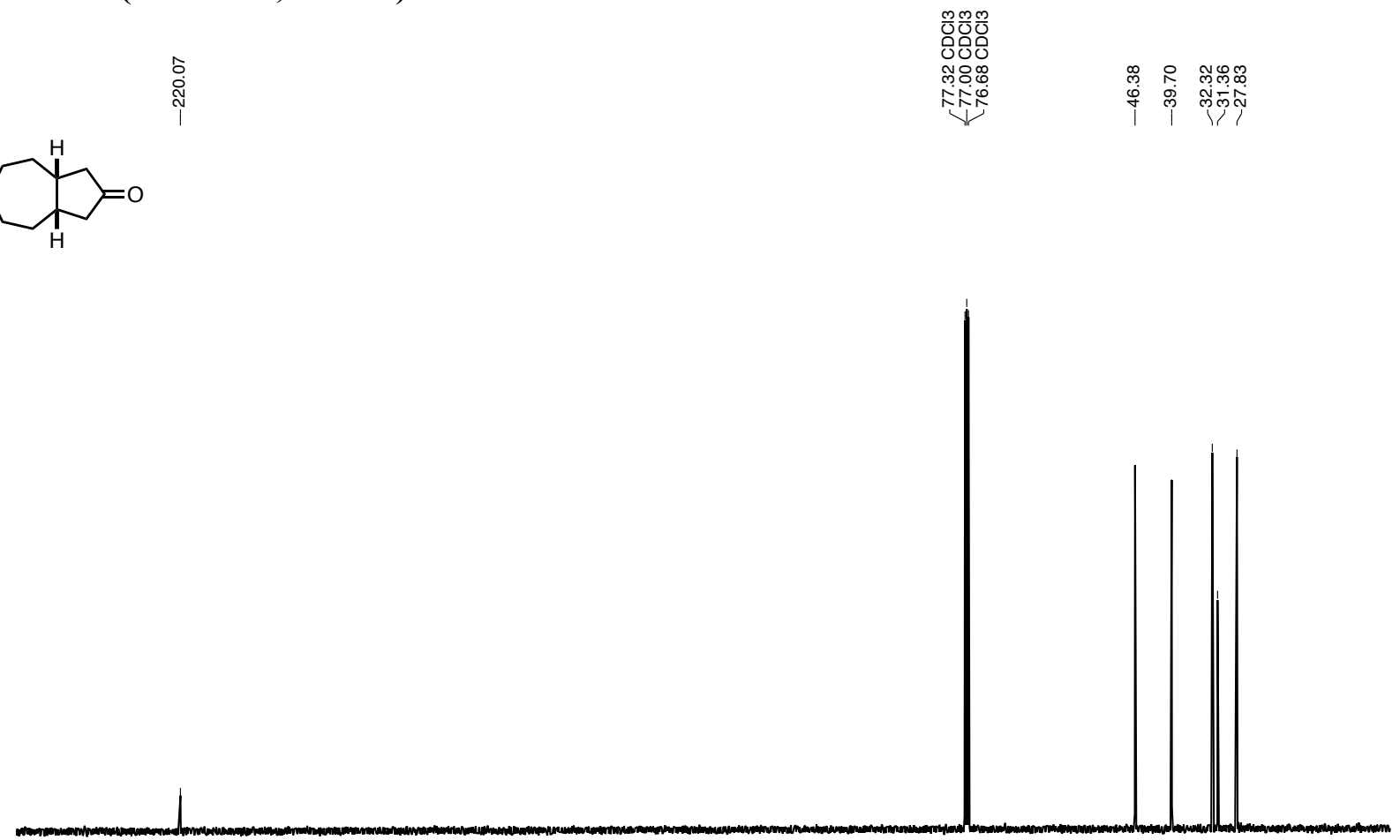

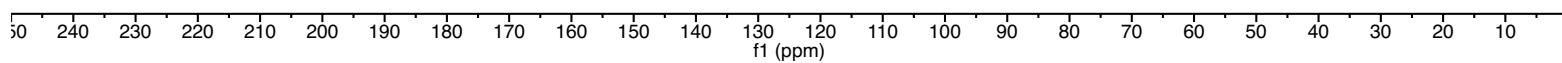


cis-Perhydroazulene (1c)

${ }^{1} \mathrm{H}$ NMR (400 $\mathrm{MHz}, \mathrm{CDCl}_{3}$ )

尊

ำ<smiles>C1CCC2CCCC2CC1</smiles>

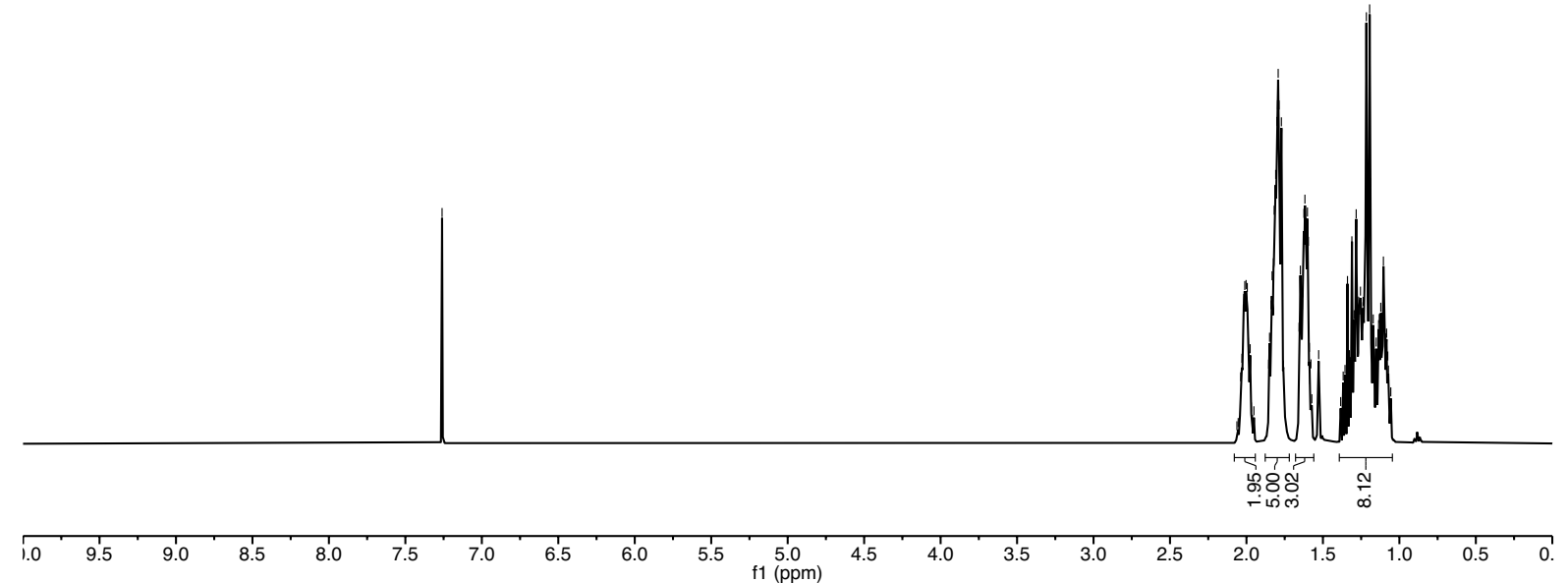

${ }^{13} \mathrm{C}$ NMR (101 MHz, $\left.\mathrm{CDCl}_{3}\right)$<smiles>C1CCC2CCCC2CC1</smiles>
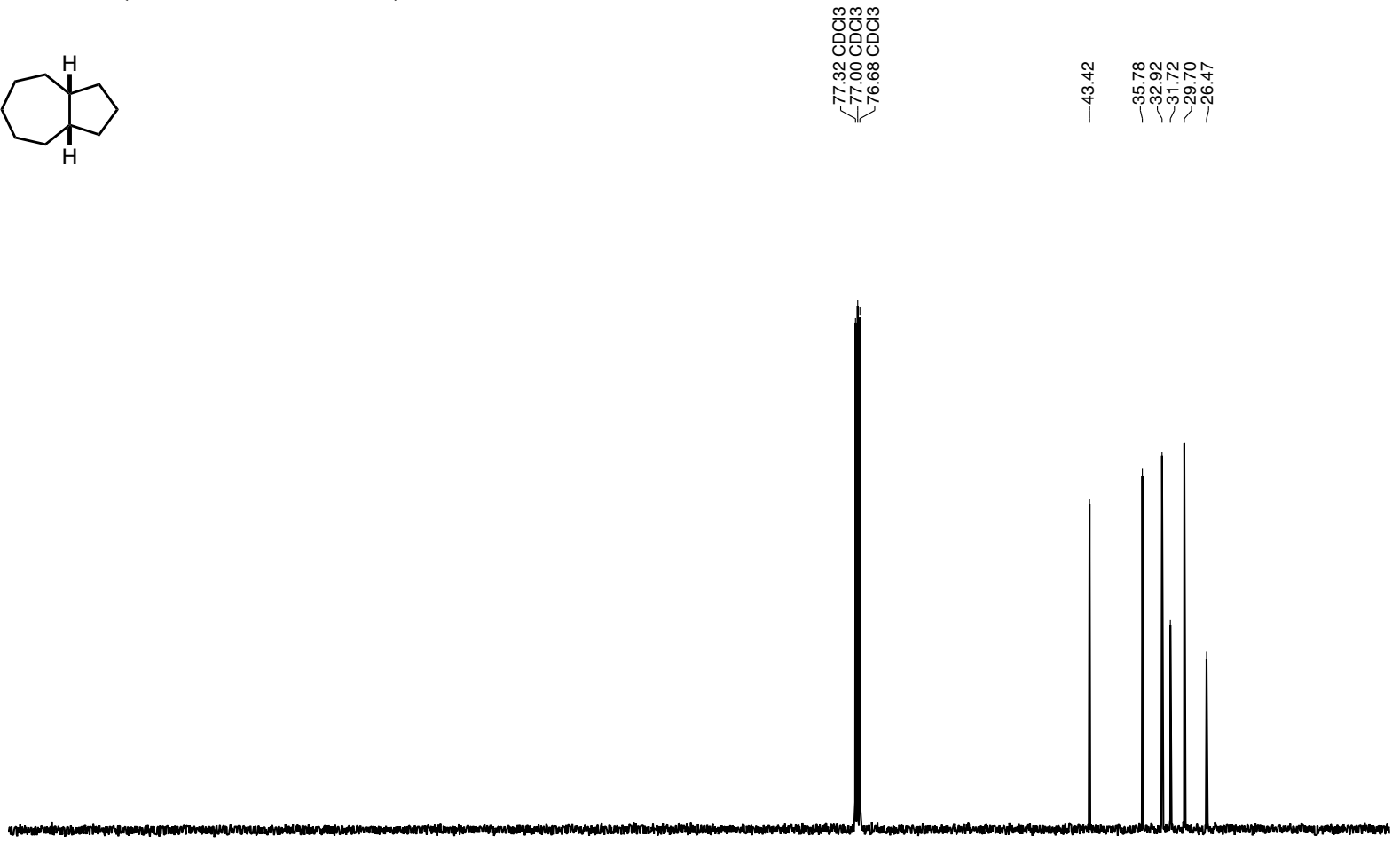

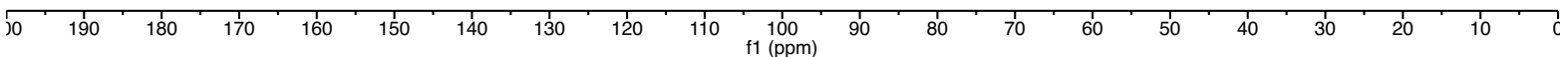


$\beta$-Hydroxy ketone 7

${ }^{1} \mathrm{H}_{\mathrm{NMR}}$ (400 $\mathrm{MHz}, \mathrm{CDCl}_{3}$ )

$\frac{m}{0}$

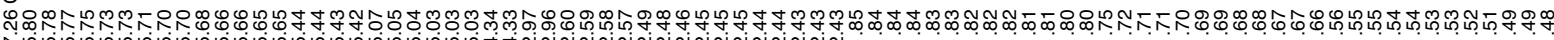<smiles>C=CC1CCCCC(=O)C1C(O)/C=C/C</smiles>

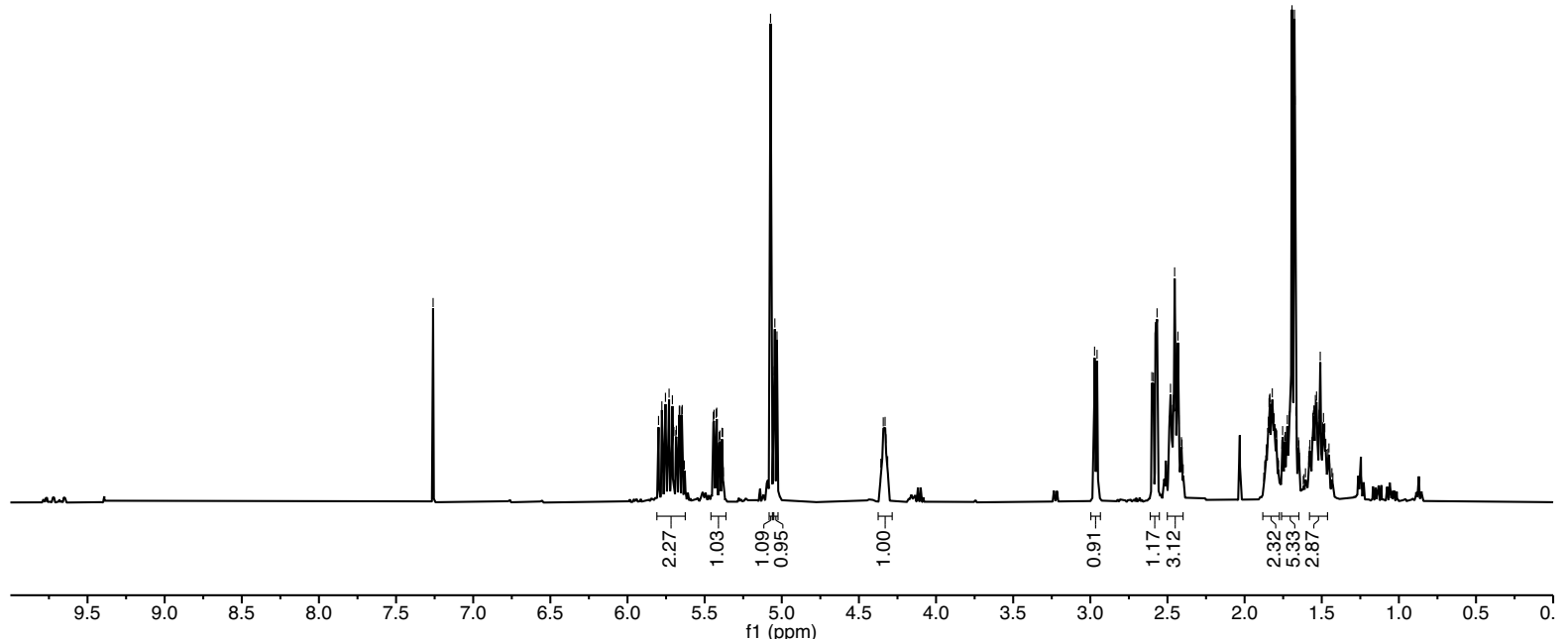

${ }^{13} \mathrm{C}$ NMR (101 MHz, $\left.\mathrm{CDCl}_{3}\right)$

$\stackrel{\oplus}{\stackrel{\oplus}{N}}$

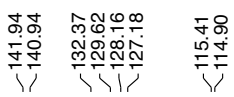

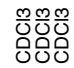

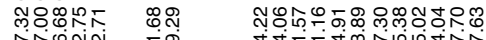

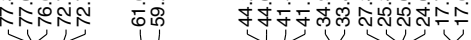<smiles>C=CC1CCCCC(=O)C1/C=C/[W]</smiles>

H

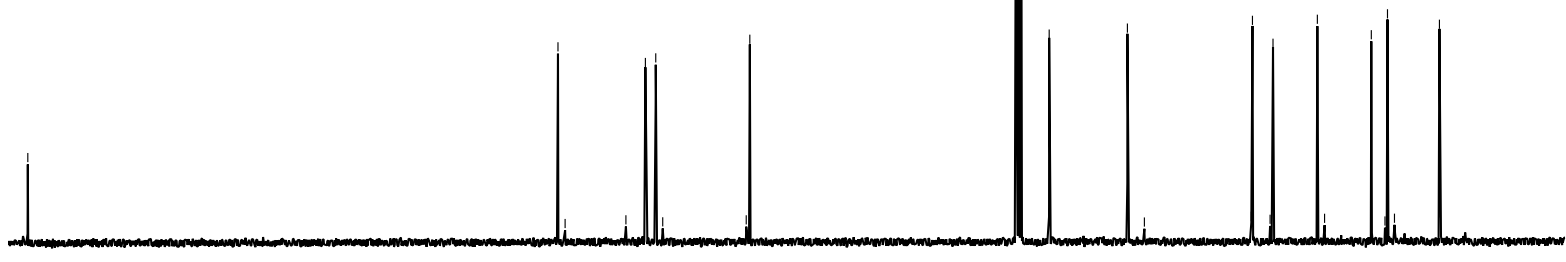


Unsaturated secondary alcohol 9

${ }^{1} \mathrm{H}$ NMR (400 MHz, $\mathrm{CDCl}_{3}$ )

응

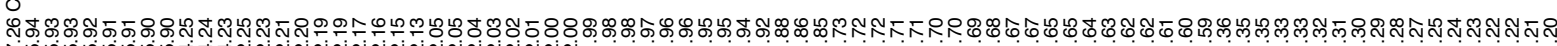<smiles>OC1CCC2CCCC=CC12</smiles>

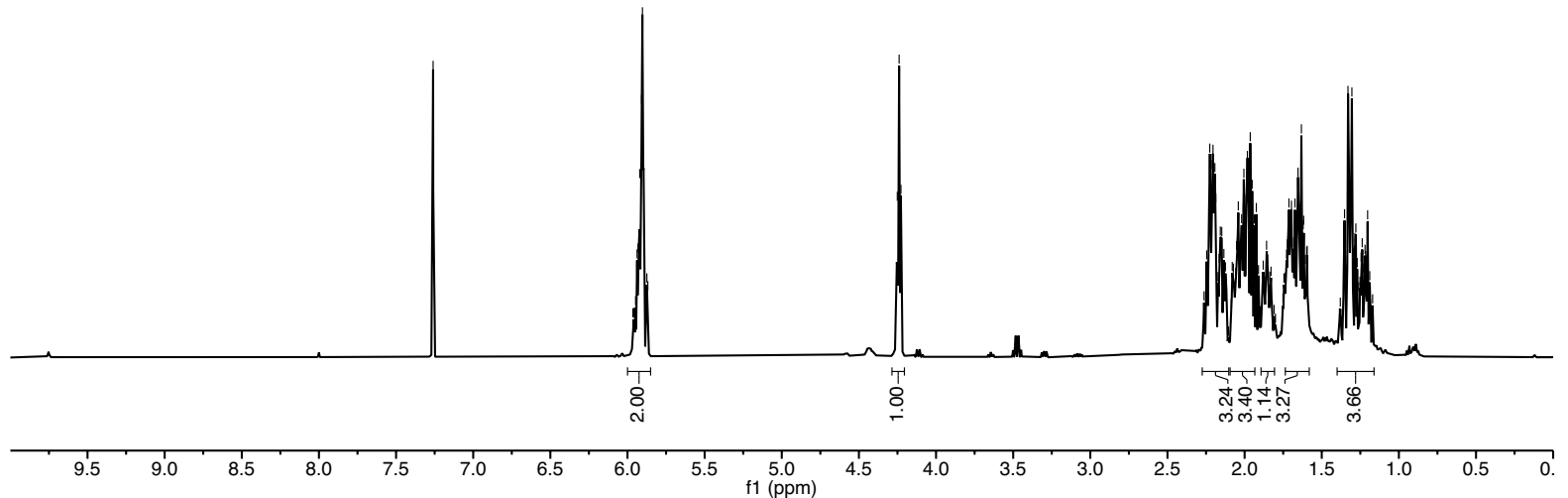

${ }^{13} \mathrm{C}$ NMR (101 MHz, $\left.\mathrm{CDCl}_{3}\right)$<smiles>OC1CCC2CCCC=CC12</smiles>

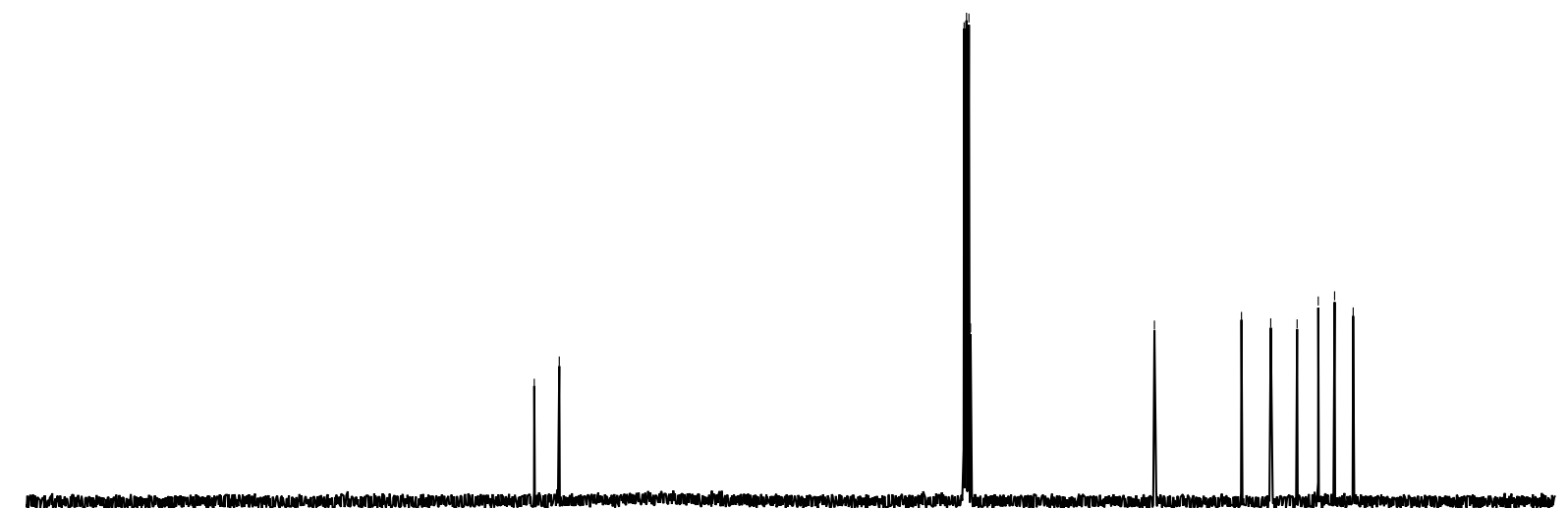

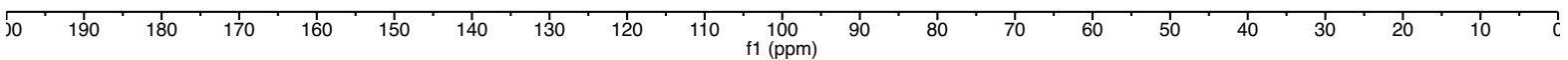


Saturated secondary alcohol $\mathbf{1 0}$

${ }^{1} \mathrm{H}$ NMR (400 MHz, $\left.\mathrm{CDCl}_{3}\right)$

$\frac{\infty}{0}$

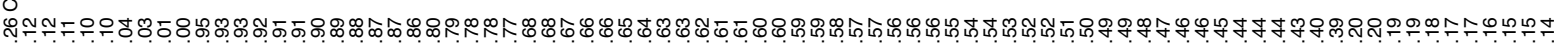

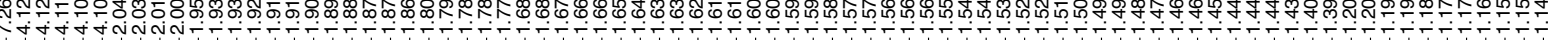<smiles>OC1CCC2CCCCCC12</smiles>

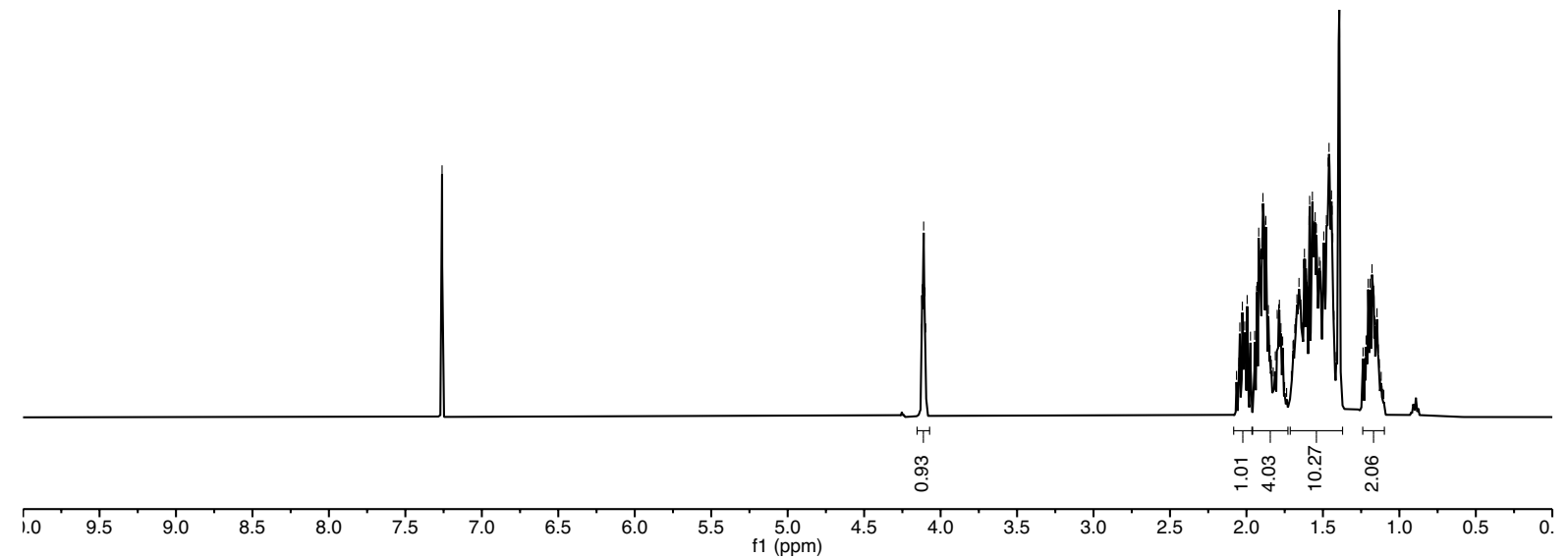

${ }^{13} \mathrm{C}$ NMR (101 MHz, $\left.\mathrm{CDCl}_{3}\right)$<smiles>OC1CCC2CCCCC12</smiles>
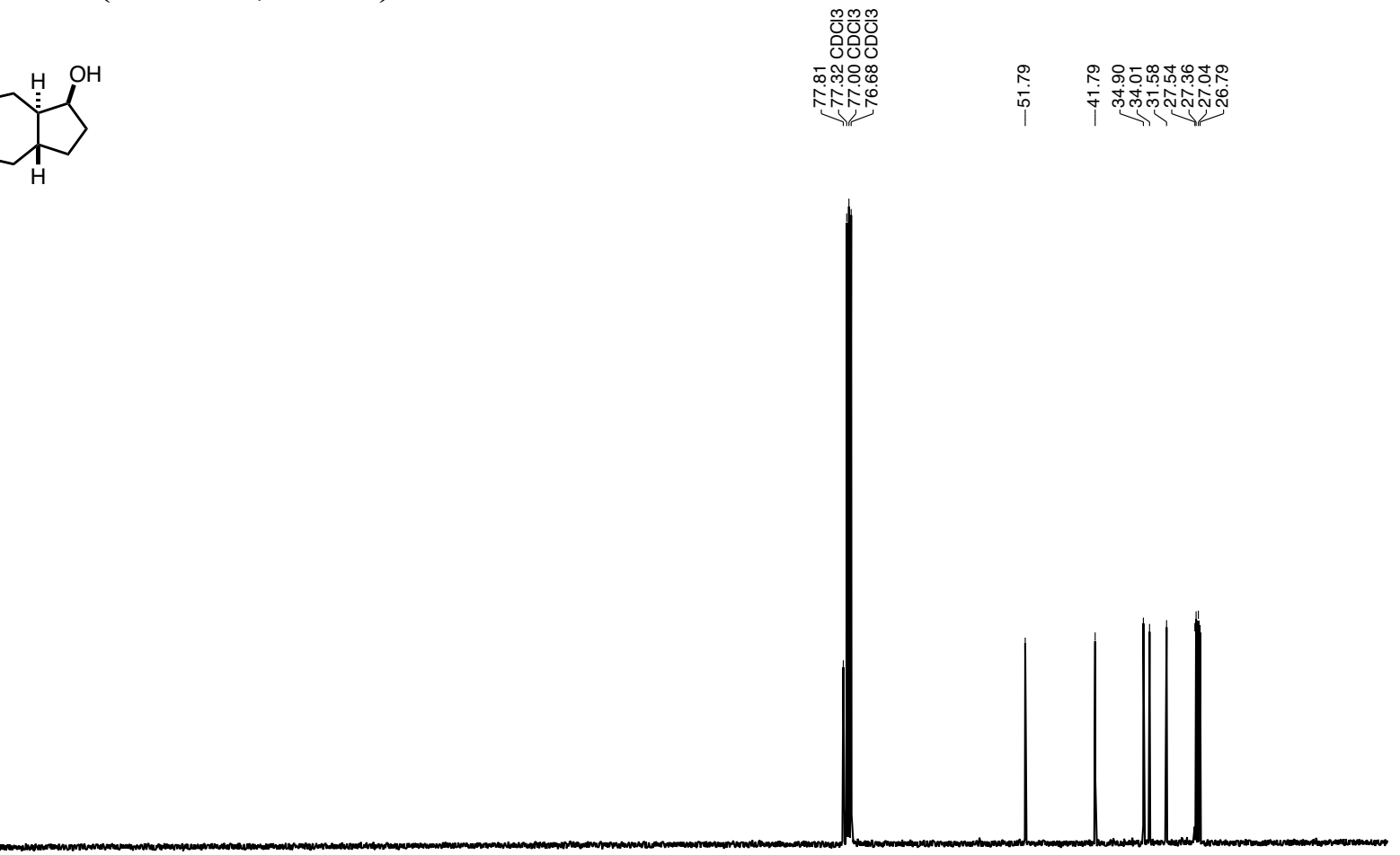

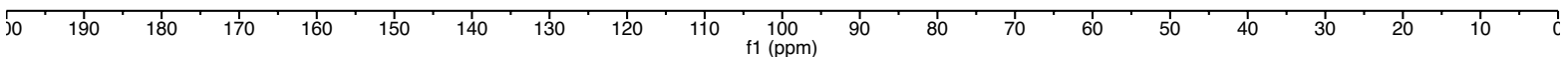


Nitrobenzoyl ester 11

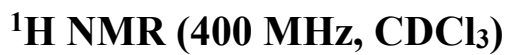

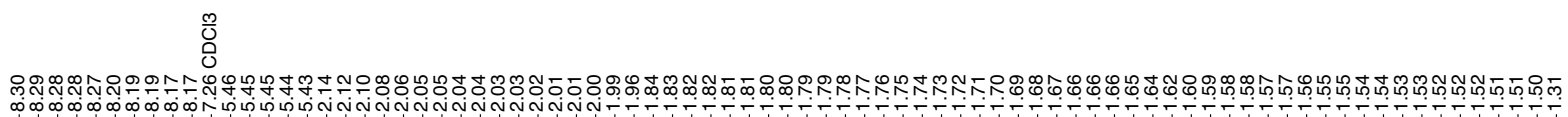<smiles>O=C(OC1CCC2CCCCC21)c1ccc([N+](=O)[O-])cc1</smiles>

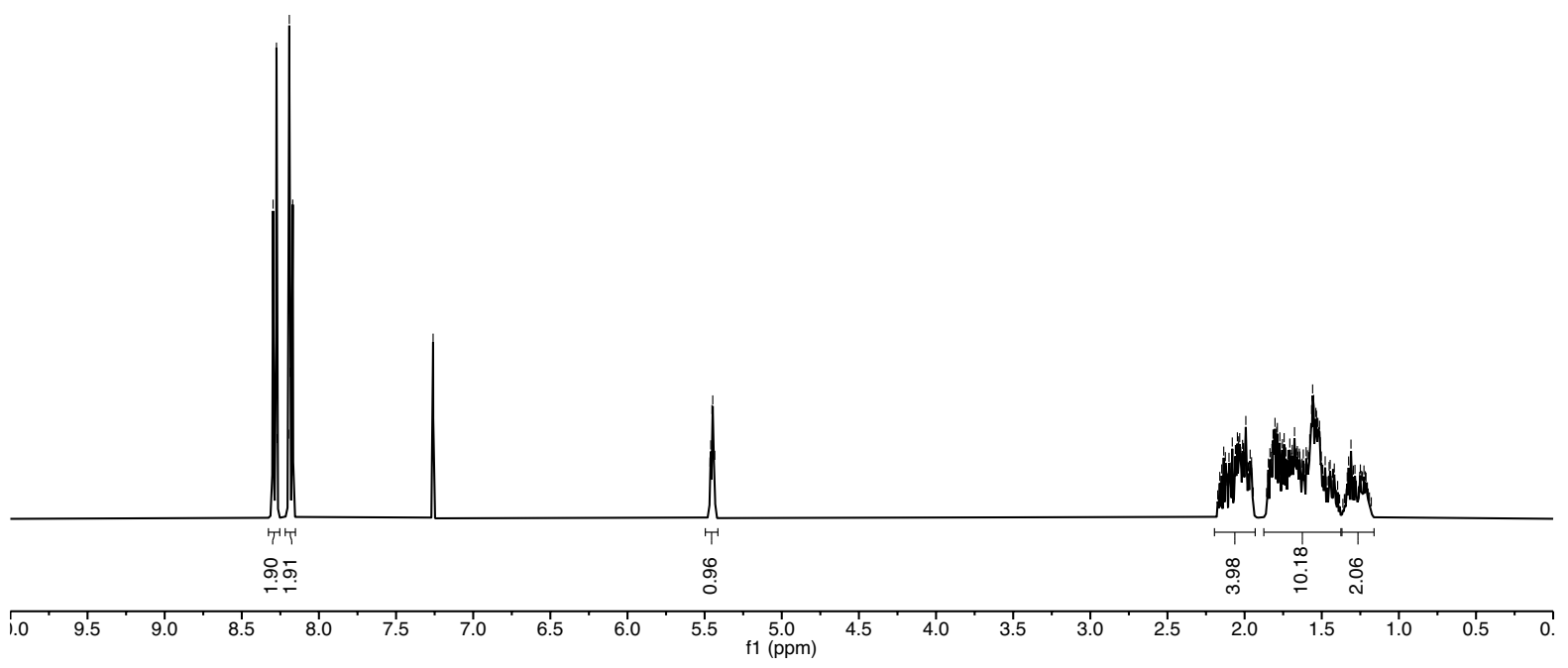

${ }^{13} \mathrm{C}$ NMR (101 MHz, $\left.\mathrm{CDCl}_{3}\right)$

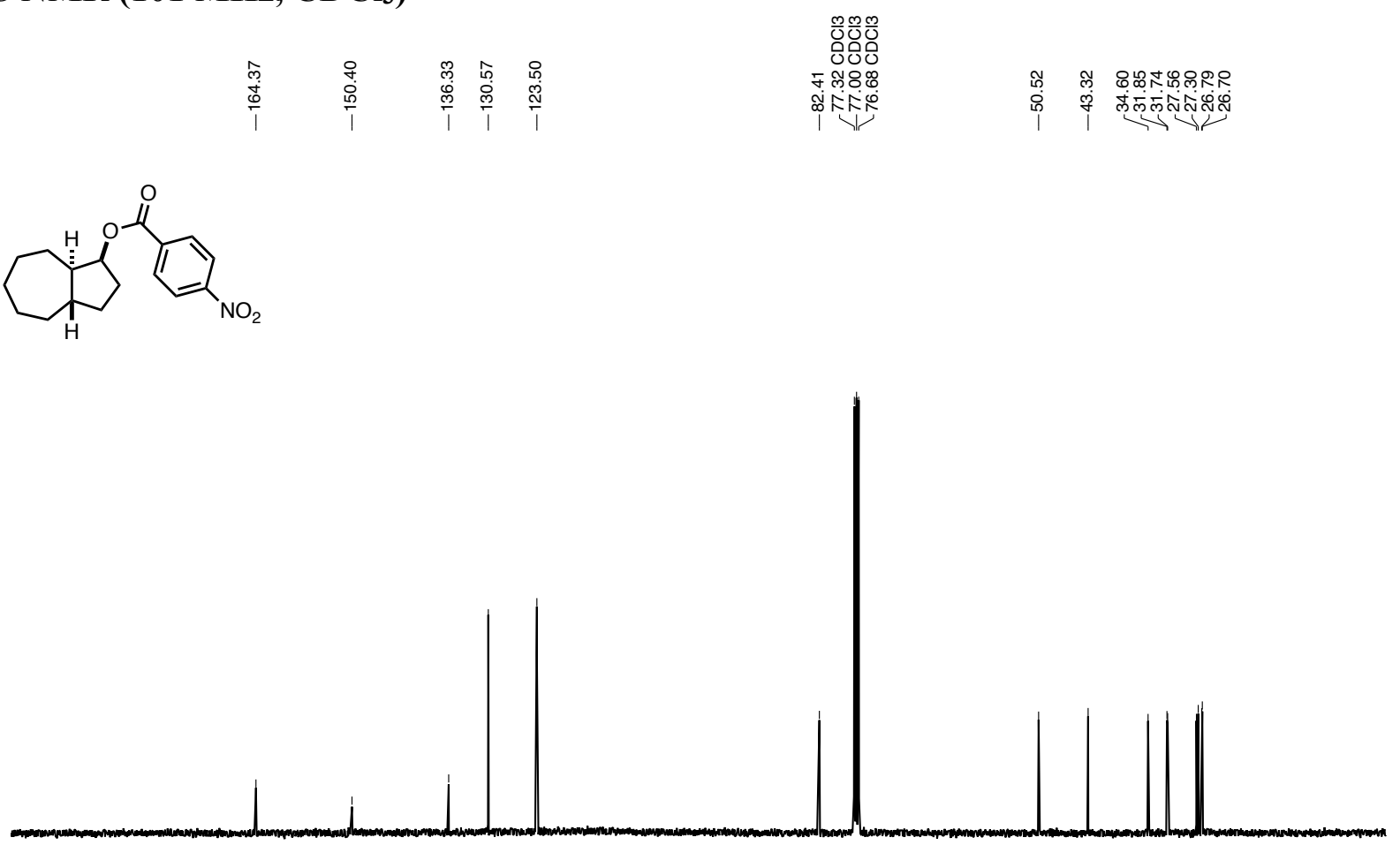

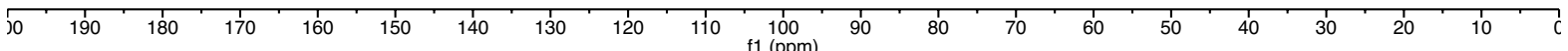


Toluate $\mathbf{1 2}$

${ }^{1} \mathrm{H}$ NMR (400 MHz, $\left.\mathrm{CDCl}_{3}\right)$

$\frac{m}{\mathrm{O}}$

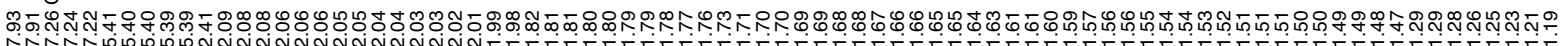<smiles>Cc1ccc(C(=O)OC2CCC3CCCCCC32)cc1</smiles>

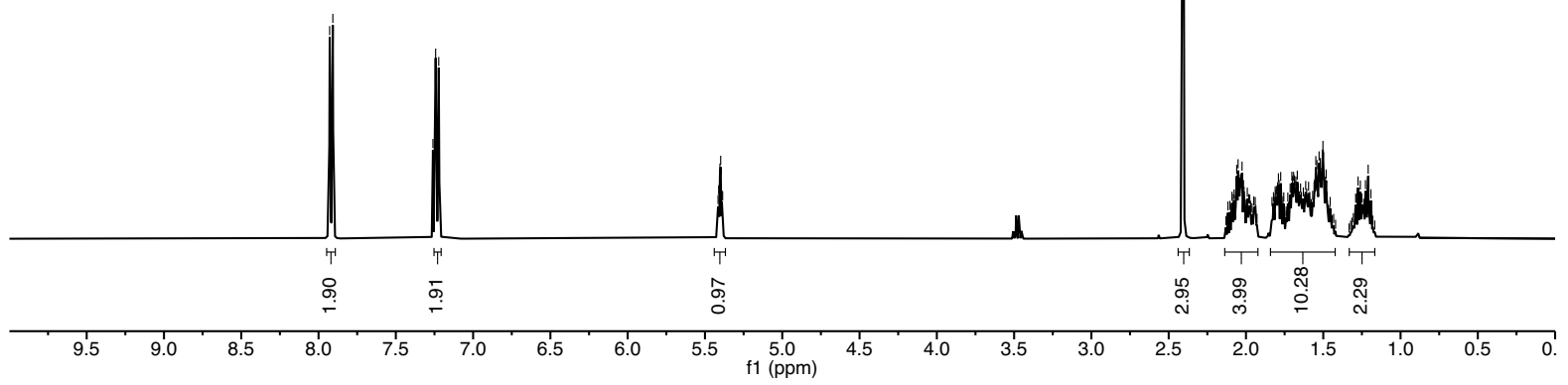

${ }^{13} \mathrm{C}$ NMR (101 MHz, $\left.\mathrm{CDCl}_{3}\right)$<smiles>Cc1ccc(C(=O)OC2CCC3CCCCCC32)cc1</smiles>
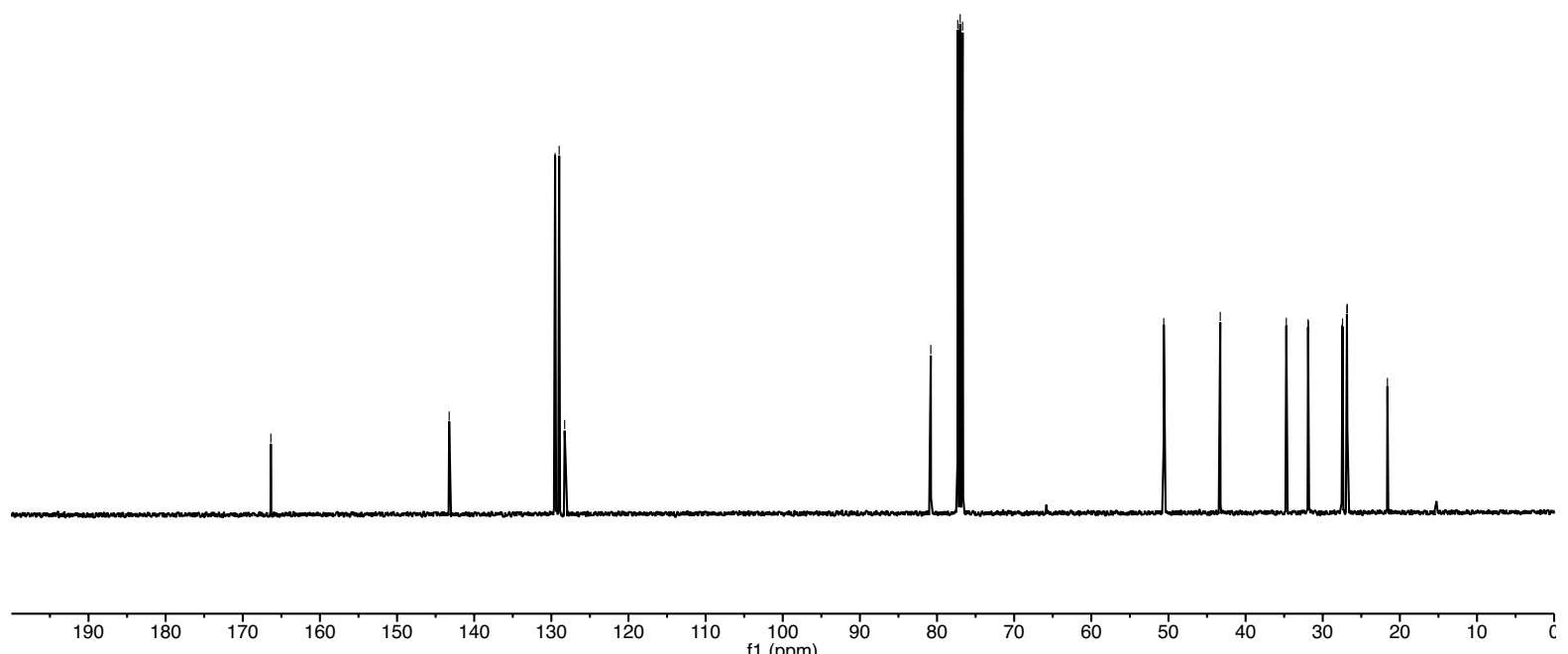
trans-Perhydroazulene (1t)

${ }^{1} \mathrm{H}$ NMR (400 MHz, $\left.\mathrm{CDCl}_{3}\right)$
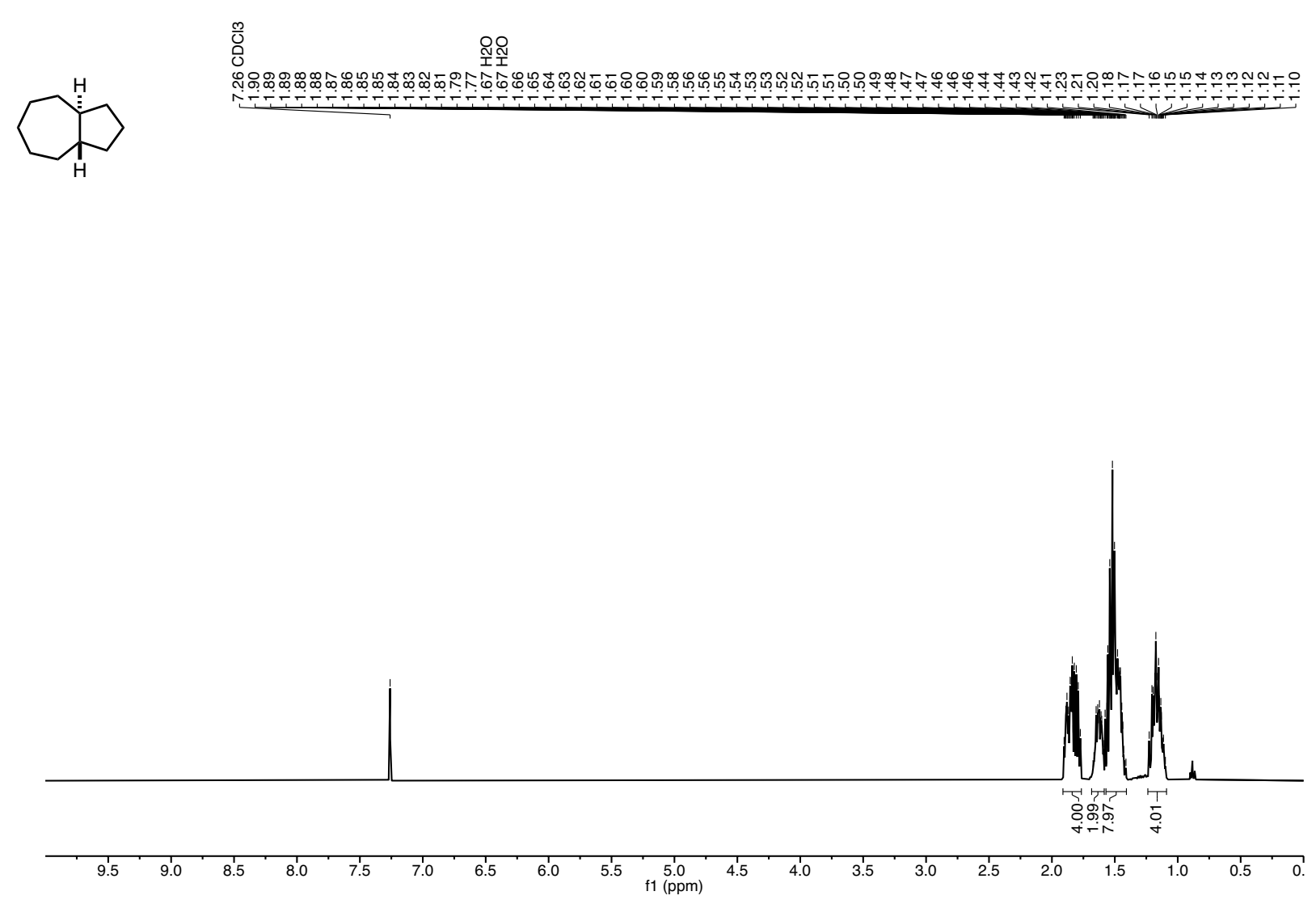

${ }^{13} \mathrm{C}$ NMR (101 MHz, $\left.\mathrm{CDCl}_{3}\right)$
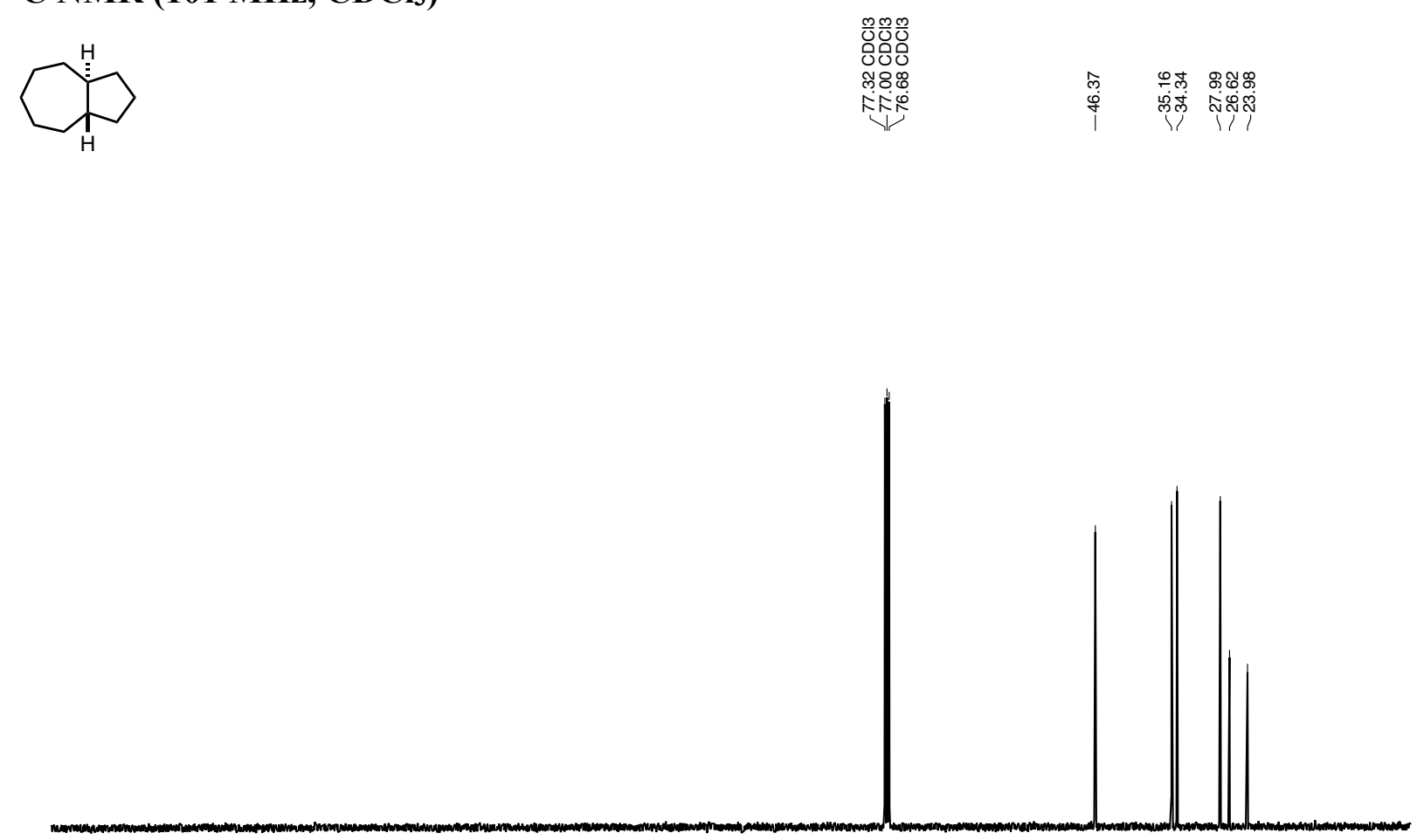

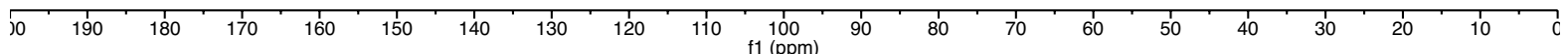




\section{X-ray Crystallographic Analysis}

\section{Experimental}

Diffraction data were collected at low temperatures (100K) using $\varphi$ - and $\omega$-scans on a BRUKER D8 Venture system equipped with dual $\mathrm{I} \mu \mathrm{S}$ microfocus sources, a PHOTON100 detector and an OXFORD CRYOSYSTEMS 700 low temperature system. Mo-K $\alpha$ radiation with wavelength $0.71073 \AA$ and a collimating Quazar multilayer mirror were used. Semi-empirical absorption correction from equivalents was applied using SADABS-2016/2 ${ }^{1}$ and the structures were solved by the dual space algorithm implemented in SHELXT2014/5. ${ }^{2}$ Refinement was performed against $F^{2}$ on all data by full-matrix least squares using SHELXL2018/3. ${ }^{3}$ All non-hydrogen atoms were refined anisotropically and $\mathrm{C}-\mathrm{H}$ hydrogen atoms were positioned at geometrically calculated positions and refined using a riding model. The isotropic displacement parameters of all hydrogen atoms were fixed to $1.2 \mathrm{x}$ the $\mathrm{U}_{\text {eq }}$ value of the atoms they are linked to.

CCDC reference number ${ }^{4}$

5: 1978700

11: 1978701

\section{Crystallization method}

5: slow evaporation (solvent: $n$-hexane)

11: slow evaporation (solvent: $\mathrm{MeOH} / \mathrm{Et}_{2} \mathrm{O}$ ) 


\section{$\underline{\text { Crystal data for } \mathbf{5}}$}

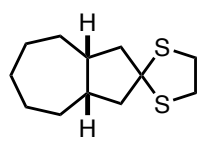

5

The structure was solved in the triclinic space group $P \overline{1}$ and the asymmetric unit contains one molecule of $\mathbf{5}$.

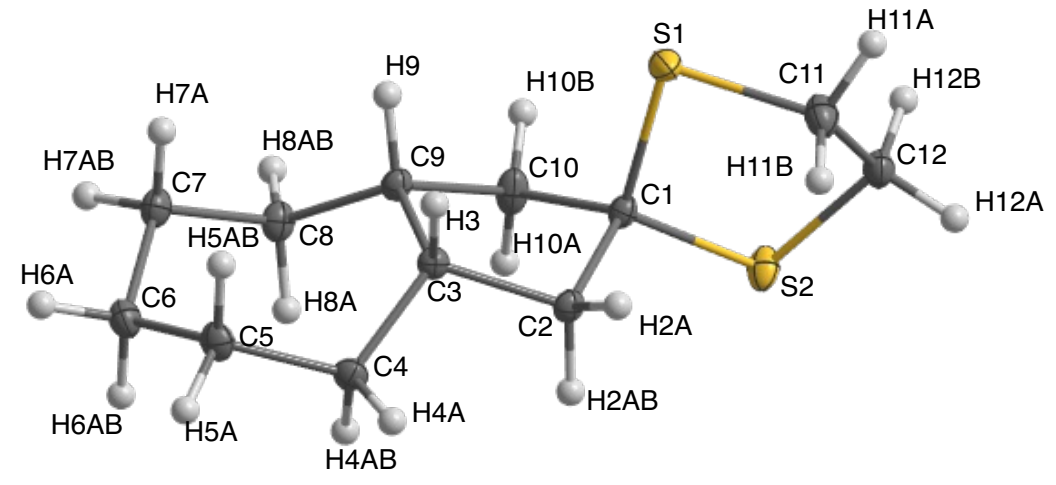

Thermal ellipsoid plot of $\mathrm{C}_{12} \mathrm{H}_{20} \mathrm{~S}_{2}(\mathbf{5})$ 
Table S1. Crystal data and structure refinement for $\mathbf{5}$.

CCDC No

Empirical formula

Formula weight

Temperature

Wavelength

Crystal system

Space group

Unit cell dimensions

Volume

Z

Density (calculated)

Absorption coefficient

$\mathrm{F}(000)$

Crystal size

Theta range for data collection

Index ranges

Reflections collected

Independent reflections

Completeness to theta $=25.242^{\circ}$

Absorption correction

Refinement method

Data / restraints / parameters

Goodness-of-fit on $F^{2}$

Final R indices $[\mathrm{I}>2 \sigma(\mathrm{I})]$

$\mathrm{R}$ indices (all data)

Largest diff. peak and hole
1978700

$\mathrm{C}_{12} \mathrm{H}_{20} \mathrm{~S}_{2}$

228.40

100(2) K

$0.71073 \AA$

Triclinic

$P \overline{1}$

$$
\begin{array}{ll}
\mathrm{a}=5.9709(3) \AA & \alpha=112.0266(17)^{\circ} . \\
\mathrm{b}=10.3213(5) \AA & \beta=103.9938(19)^{\circ} . \\
\mathrm{c}=11.0614(6) \AA & \gamma=99.262(2)^{\circ} .
\end{array}
$$

$588.69(5) \AA^{3}$

2

$1.289 \mathrm{Mg} / \mathrm{m}^{3}$

$0.413 \mathrm{~mm}^{-1}$

248

$0.530 \times 0.343 \times 0.153 \mathrm{~mm}^{3}$

2.217 to $31.503^{\circ}$.

$-8<=\mathrm{h}<=8,-15<=\mathrm{k}<=15,-16<=1<=16$

33917

$3922[\mathrm{R}($ int $)=0.0443]$

$99.9 \%$

Semi-empirical from equivalents

Full-matrix least-squares on $F^{2}$

3922 / 0 / 127

1.047

$\mathrm{R}_{1}=0.0333, \mathrm{wR}_{2}=0.0777$

$\mathrm{R}_{1}=0.0437, \mathrm{wR}_{2}=0.0815$

0.528 and -0.304 e. $\AA^{-3}$ 
Table S2. Atomic coordinates (x 10 $)$ and equivalent isotropic displacement parameters $\left(\AA^{2} \times 10^{3}\right)$ for 5 . $U(e q)$ is defined as one third of the trace of the orthogonalized $U^{i j}$ tensor.

\begin{tabular}{lcccc}
\hline & $\mathrm{x}$ & $\mathrm{y}$ & $\mathrm{z}$ & $\mathrm{U}(\mathrm{eq})$ \\
\hline $\mathrm{S}(1)$ & & & \\
$\mathrm{S}(2)$ & $6815(1)$ & $5898(1)$ & $4015(1)$ & $18(1)$ \\
$\mathrm{C}(1)$ & $6049(1)$ & $8516(1)$ & $5951(1)$ & $17(1)$ \\
$\mathrm{C}(2)$ & $5916(2)$ & $7538(1)$ & $4153(1)$ & $11(1)$ \\
$\mathrm{C}(3)$ & $7463(2)$ & $8456(1)$ & $3684(1)$ & $12(1)$ \\
$\mathrm{C}(4)$ & $6491(2)$ & $7681(1)$ & $2093(1)$ & $10(1)$ \\
$\mathrm{C}(5)$ & $6915(2)$ & $8771(1)$ & $1486(1)$ & $13(1)$ \\
$\mathrm{C}(6)$ & $6321(2)$ & $8104(1)$ & $-88(1)$ & $15(1)$ \\
$\mathrm{C}(7)$ & $3669(2)$ & $7728(1)$ & $-910(1)$ & $16(1)$ \\
$\mathrm{C}(8)$ & $1986(2)$ & $6572(1)$ & $-745(1)$ & $16(1)$ \\
$\mathrm{C}(9)$ & $1861(2)$ & $7016(1)$ & $719(1)$ & $14(1)$ \\
$\mathrm{C}(10)$ & $3835(2)$ & $6782(1)$ & $1714(1)$ & $12(1)$ \\
$\mathrm{C}(11)$ & $3416(2)$ & $7145(1)$ & $3113(1)$ & $16(1)$ \\
$\mathrm{C}(12)$ & $9010(2)$ & $6764(1)$ & $5728(1)$ & $19(1)$ \\
\hline
\end{tabular}


Table S3. Bond lengths $[\AA]$ and angles $\left[^{\circ}\right]$ for 5.

\begin{tabular}{|c|c|c|c|}
\hline $\mathrm{S}(1)-\mathrm{C}(11)$ & $1.8053(12)$ & $\mathrm{C}(12)-\mathrm{S}(2)-\mathrm{C}(1)$ & $98.91(5)$ \\
\hline$S(1)-C(1)$ & $1.8211(11)$ & $C(2)-C(1)-C(10)$ & $101.84(8)$ \\
\hline$S(2)-C(12)$ & $1.8173(12)$ & $C(2)-C(1)-S(1)$ & $111.78(7)$ \\
\hline$S(2)-C(1)$ & $1.8369(10)$ & $\mathrm{C}(10)-\mathrm{C}(1)-\mathrm{S}(1)$ & $110.39(8)$ \\
\hline $\mathrm{C}(1)-\mathrm{C}(2)$ & $1.5293(14)$ & $C(2)-C(1)-S(2)$ & $113.25(7)$ \\
\hline$C(1)-C(10)$ & $1.5293(15)$ & $\mathrm{C}(10)-\mathrm{C}(1)-\mathrm{S}(2)$ & $112.82(7)$ \\
\hline$C(2)-C(3)$ & $1.5409(14)$ & $\mathrm{S}(1)-\mathrm{C}(1)-\mathrm{S}(2)$ & $106.83(5)$ \\
\hline $\mathrm{C}(2)-\mathrm{H}(2 \mathrm{~A})$ & 0.9900 & $C(1)-C(2)-C(3)$ & $105.14(8)$ \\
\hline $\mathrm{C}(2)-\mathrm{H}(2 \mathrm{AB})$ & 0.9900 & $\mathrm{C}(1)-\mathrm{C}(2)-\mathrm{H}(2 \mathrm{~A})$ & 110.7 \\
\hline$C(3)-C(4)$ & $1.5288(14)$ & $\mathrm{C}(3)-\mathrm{C}(2)-\mathrm{H}(2 \mathrm{~A})$ & 110.7 \\
\hline$C(3)-C(9)$ & $1.5699(14)$ & $\mathrm{C}(1)-\mathrm{C}(2)-\mathrm{H}(2 \mathrm{AB})$ & 110.7 \\
\hline $\mathrm{C}(3)-\mathrm{H}(3)$ & 1.0000 & $\mathrm{C}(3)-\mathrm{C}(2)-\mathrm{H}(2 \mathrm{AB})$ & 110.7 \\
\hline$C(4)-C(5)$ & $1.5345(15)$ & $\mathrm{H}(2 \mathrm{~A})-\mathrm{C}(2)-\mathrm{H}(2 \mathrm{AB})$ & 108.8 \\
\hline $\mathrm{C}(4)-\mathrm{H}(4 \mathrm{~A})$ & 0.9900 & $\mathrm{C}(4)-\mathrm{C}(3)-\mathrm{C}(2)$ & $110.55(8)$ \\
\hline $\mathrm{C}(4)-\mathrm{H}(4 \mathrm{AB})$ & 0.9900 & $\mathrm{C}(4)-\mathrm{C}(3)-\mathrm{C}(9)$ & $118.02(9)$ \\
\hline$C(5)-C(6)$ & $1.5251(16)$ & $C(2)-C(3)-C(9)$ & $105.28(8)$ \\
\hline $\mathrm{C}(5)-\mathrm{H}(5 \mathrm{~A})$ & 0.9900 & $\mathrm{C}(4)-\mathrm{C}(3)-\mathrm{H}(3)$ & 107.5 \\
\hline $\mathrm{C}(5)-\mathrm{H}(5 \mathrm{AB})$ & 0.9900 & $\mathrm{C}(2)-\mathrm{C}(3)-\mathrm{H}(3)$ & 107.5 \\
\hline$C(6)-C(7)$ & $1.5278(16)$ & $\mathrm{C}(9)-\mathrm{C}(3)-\mathrm{H}(3)$ & 107.5 \\
\hline $\mathrm{C}(6)-\mathrm{H}(6 \mathrm{~A})$ & 0.9900 & $\mathrm{C}(3)-\mathrm{C}(4)-\mathrm{C}(5)$ & $115.44(9)$ \\
\hline $\mathrm{C}(6)-\mathrm{H}(6 \mathrm{AB})$ & 0.9900 & $\mathrm{C}(3)-\mathrm{C}(4)-\mathrm{H}(4 \mathrm{~A})$ & 108.4 \\
\hline $\mathrm{C}(7)-\mathrm{C}(8)$ & $1.5329(15)$ & $\mathrm{C}(5)-\mathrm{C}(4)-\mathrm{H}(4 \mathrm{~A})$ & 108.4 \\
\hline $\mathrm{C}(7)-\mathrm{H}(7 \mathrm{~A})$ & 0.9900 & $\mathrm{C}(3)-\mathrm{C}(4)-\mathrm{H}(4 \mathrm{AB})$ & 108.4 \\
\hline $\mathrm{C}(7)-\mathrm{H}(7 \mathrm{AB})$ & 0.9900 & $\mathrm{C}(5)-\mathrm{C}(4)-\mathrm{H}(4 \mathrm{AB})$ & 108.4 \\
\hline$C(8)-C(9)$ & $1.5244(15)$ & $\mathrm{H}(4 \mathrm{~A})-\mathrm{C}(4)-\mathrm{H}(4 \mathrm{AB})$ & 107.5 \\
\hline $\mathrm{C}(8)-\mathrm{H}(8 \mathrm{~A})$ & 0.9900 & $C(6)-C(5)-C(4)$ & $114.80(9)$ \\
\hline $\mathrm{C}(8)-\mathrm{H}(8 \mathrm{AB})$ & 0.9900 & $\mathrm{C}(6)-\mathrm{C}(5)-\mathrm{H}(5 \mathrm{~A})$ & 108.6 \\
\hline $\mathrm{C}(9)-\mathrm{C}(10)$ & $1.5431(15)$ & $\mathrm{C}(4)-\mathrm{C}(5)-\mathrm{H}(5 \mathrm{~A})$ & 108.6 \\
\hline $\mathrm{C}(9)-\mathrm{H}(9)$ & 1.0000 & $\mathrm{C}(6)-\mathrm{C}(5)-\mathrm{H}(5 \mathrm{AB})$ & 108.6 \\
\hline $\mathrm{C}(10)-\mathrm{H}(10 \mathrm{~A})$ & 0.9900 & $\mathrm{C}(4)-\mathrm{C}(5)-\mathrm{H}(5 \mathrm{AB})$ & 108.6 \\
\hline $\mathrm{C}(10)-\mathrm{H}(10 \mathrm{~B})$ & 0.9900 & $\mathrm{H}(5 \mathrm{~A})-\mathrm{C}(5)-\mathrm{H}(5 \mathrm{AB})$ & 107.5 \\
\hline $\mathrm{C}(11)-\mathrm{C}(12)$ & $1.5199(16)$ & $C(5)-C(6)-C(7)$ & $115.15(9)$ \\
\hline $\mathrm{C}(11)-\mathrm{H}(11 \mathrm{~A})$ & 0.9900 & $\mathrm{C}(5)-\mathrm{C}(6)-\mathrm{H}(6 \mathrm{~A})$ & 108.5 \\
\hline $\mathrm{C}(11)-\mathrm{H}(11 \mathrm{~B})$ & 0.9900 & $\mathrm{C}(7)-\mathrm{C}(6)-\mathrm{H}(6 \mathrm{~A})$ & 108.5 \\
\hline $\mathrm{C}(12)-\mathrm{H}(12 \mathrm{~A})$ & 0.9900 & $\mathrm{C}(5)-\mathrm{C}(6)-\mathrm{H}(6 \mathrm{AB})$ & 108.5 \\
\hline \multirow[t]{2}{*}{$\mathrm{C}(12)-\mathrm{H}(12 \mathrm{~B})$} & 0.9900 & $\mathrm{C}(7)-\mathrm{C}(6)-\mathrm{H}(6 \mathrm{AB})$ & 108.5 \\
\hline & & $H(6 A)-C(6)-H(6 A B)$ & 107.5 \\
\hline $\mathrm{C}(11)-\mathrm{S}(1)-\mathrm{C}(1)$ & $94.16(5)$ & $C(6)-C(7)-C(8)$ & $114.59(9)$ \\
\hline
\end{tabular}




$\begin{array}{llll}\mathrm{C}(6)-\mathrm{C}(7)-\mathrm{H}(7 \mathrm{~A}) & 108.6 & \mathrm{C}(1)-\mathrm{C}(10)-\mathrm{H}(10 \mathrm{~A}) & 110.8 \\ \mathrm{C}(8)-\mathrm{C}(7)-\mathrm{H}(7 \mathrm{~A}) & 108.6 & \mathrm{C}(9)-\mathrm{C}(10)-\mathrm{H}(10 \mathrm{~A}) & 110.8 \\ \mathrm{C}(6)-\mathrm{C}(7)-\mathrm{H}(7 \mathrm{AB}) & 108.6 & \mathrm{C}(1)-\mathrm{C}(10)-\mathrm{H}(10 \mathrm{~B}) & 110.8 \\ \mathrm{C}(8)-\mathrm{C}(7)-\mathrm{H}(7 \mathrm{AB}) & 108.6 & \mathrm{C}(9)-\mathrm{C}(10)-\mathrm{H}(10 \mathrm{~B}) & 110.8 \\ \mathrm{H}(7 \mathrm{~A})-\mathrm{C}(7)-\mathrm{H}(7 \mathrm{AB}) & 107.6 & \mathrm{H}(10 \mathrm{~A})-\mathrm{C}(10)-\mathrm{H}(10 \mathrm{~B}) & 108.9 \\ \mathrm{C}(9)-\mathrm{C}(8)-\mathrm{C}(7) & 115.58(9) & \mathrm{C}(12)-\mathrm{C}(11)-\mathrm{S}(1) & 107.39(8) \\ \mathrm{C}(9)-\mathrm{C}(8)-\mathrm{H}(8 \mathrm{~A}) & 108.4 & \mathrm{C}(12)-\mathrm{C}(11)-\mathrm{H}(11 \mathrm{~A}) & 110.2 \\ \mathrm{C}(7)-\mathrm{C}(8)-\mathrm{H}(8 \mathrm{~A}) & 108.4 & \mathrm{~S}(1)-\mathrm{C}(11)-\mathrm{H}(11 \mathrm{~A}) & 110.2 \\ \mathrm{C}(9)-\mathrm{C}(8)-\mathrm{H}(8 \mathrm{AB}) & 108.4 & \mathrm{C}(12)-\mathrm{C}(11)-\mathrm{H}(11 \mathrm{~B}) & 110.2 \\ \mathrm{C}(7)-\mathrm{C}(8)-\mathrm{H}(8 \mathrm{AB}) & 108.4 & \mathrm{~S}(1)-\mathrm{C}(11)-\mathrm{H}(11 \mathrm{~B}) & 110.2 \\ \mathrm{H}(8 \mathrm{~A})-\mathrm{C}(8)-\mathrm{H}(8 \mathrm{AB}) & 107.4 & \mathrm{H}(11 \mathrm{~A})-\mathrm{C}(11)-\mathrm{H}(11 \mathrm{~B}) & 108.5 \\ \mathrm{C}(8)-\mathrm{C}(9)-\mathrm{C}(10) & 111.11(9) & \mathrm{C}(11)-\mathrm{C}(12)-\mathrm{S}(2) & 108.47(8) \\ \mathrm{C}(8)-\mathrm{C}(9)-\mathrm{C}(3) & 117.51(9) & \mathrm{C}(11)-\mathrm{C}(12)-\mathrm{H}(12 \mathrm{~A}) & 110.0 \\ \mathrm{C}(10)-\mathrm{C}(9)-\mathrm{C}(3) & 104.93(8) & \mathrm{S}(2)-\mathrm{C}(12)-\mathrm{H}(12 \mathrm{~A}) & 110.0 \\ \mathrm{C}(8)-\mathrm{C}(9)-\mathrm{H}(9) & 107.6 & \mathrm{C}(11)-\mathrm{C}(12)-\mathrm{H}(12 \mathrm{~B}) & 110.0 \\ \mathrm{C}(10)-\mathrm{C}(9)-\mathrm{H}(9) & 107.6 & \mathrm{~S}(2)-\mathrm{C}(12)-\mathrm{H}(12 \mathrm{~B}) & 110.0 \\ \mathrm{C}(3)-\mathrm{C}(9)-\mathrm{H}(9) & 107.6 & \mathrm{H}(12 \mathrm{~A})-\mathrm{C}(12)-\mathrm{H}(12 \mathrm{~B}) & 108.4 \\ \mathrm{C}(1)-\mathrm{C}(10)-\mathrm{C}(9) & 104.67(8) & & \end{array}$


Table S4. Anisotropic displacement parameters $\left(\AA^{2} \times 10^{3}\right)$ for $\mathbf{5}$. The anisotropic displacement factor exponent takes the form: $-2 \pi^{2}\left[\mathrm{~h}^{2} \mathrm{a}^{* 2} \mathrm{U}^{11}+\ldots+2 \mathrm{hk} \mathrm{a} \mathrm{a}^{*} \mathrm{U}^{12}\right]$

\begin{tabular}{lcccccc}
\hline & $\mathrm{U}^{11}$ & $\mathrm{U}^{22}$ & $\mathrm{U}^{33}$ & $\mathrm{U}^{23}$ & $\mathrm{U}^{13}$ & $\mathrm{U}^{12}$ \\
\hline $\mathrm{S}(1)$ & $31(1)$ & $13(1)$ & $12(1)$ & $5(1)$ & $7(1)$ & $8(1)$ \\
$\mathrm{S}(2)$ & $18(1)$ & $22(1)$ & $11(1)$ & $5(1)$ & $7(1)$ & $11(1)$ \\
$\mathrm{C}(1)$ & $11(1)$ & $13(1)$ & $10(1)$ & $4(1)$ & $4(1)$ & $3(1)$ \\
$\mathrm{C}(2)$ & $9(1)$ & $13(1)$ & $12(1)$ & $5(1)$ & $3(1)$ & $2(1)$ \\
$\mathrm{C}(3)$ & $8(1)$ & $12(1)$ & $12(1)$ & $5(1)$ & $4(1)$ & $3(1)$ \\
$\mathrm{C}(4)$ & $13(1)$ & $13(1)$ & $14(1)$ & $6(1)$ & $5(1)$ & $2(1)$ \\
$\mathrm{C}(5)$ & $16(1)$ & $18(1)$ & $14(1)$ & $9(1)$ & $7(1)$ & $5(1)$ \\
$\mathrm{C}(6)$ & $18(1)$ & $19(1)$ & $14(1)$ & $9(1)$ & $5(1)$ & $7(1)$ \\
$\mathrm{C}(7)$ & $14(1)$ & $18(1)$ & $12(1)$ & $6(1)$ & $2(1)$ & $4(1)$ \\
$\mathrm{C}(8)$ & $10(1)$ & $19(1)$ & $14(1)$ & $7(1)$ & $4(1)$ & $5(1)$ \\
$\mathrm{C}(9)$ & $10(1)$ & $13(1)$ & $12(1)$ & $5(1)$ & $3(1)$ & $2(1)$ \\
$\mathrm{C}(10)$ & $9(1)$ & $25(1)$ & $12(1)$ & $8(1)$ & $4(1)$ & $1(1)$ \\
$\mathrm{C}(11)$ & $23(1)$ & $23(1)$ & $15(1)$ & $10(1)$ & $6(1)$ & $12(1)$ \\
$\mathrm{C}(12)$ & $17(1)$ & $18(1)$ & $11(1)$ & $6(1)$ & $4(1)$ & $4(1)$ \\
& & & & & & \\
\hline
\end{tabular}


Table S5. Hydrogen coordinates $\left(\mathrm{x} 10^{4}\right)$ and isotropic displacement parameters $\left(\AA^{2} \times 10^{3}\right)$ for 5 .

\begin{tabular}{|c|c|c|c|c|}
\hline & $\mathrm{x}$ & $\mathrm{y}$ & $\mathrm{z}$ & $\mathrm{U}(\mathrm{eq})$ \\
\hline $\mathrm{H}(2 \mathrm{~A})$ & 9177 & 8499 & 4034 & 14 \\
\hline $\mathrm{H}(2 \mathrm{AB})$ & 7307 & 9461 & 4022 & 14 \\
\hline $\mathrm{H}(3)$ & 7427 & 6971 & 1789 & 12 \\
\hline $\mathrm{H}(4 \mathrm{~A})$ & 8624 & 9348 & 1922 & 16 \\
\hline $\mathrm{H}(4 \mathrm{AB})$ & 5933 & 9452 & 1739 & 16 \\
\hline $\mathrm{H}(5 \mathrm{~A})$ & 7260 & 8797 & -327 & 18 \\
\hline $\mathrm{H}(5 \mathrm{AB})$ & 6850 & 7207 & -378 & 18 \\
\hline $\mathrm{H}(6 \mathrm{~A})$ & 3496 & 7384 & -1901 & 19 \\
\hline $\mathrm{H}(6 \mathrm{AB})$ & 3147 & 8627 & -626 & 19 \\
\hline $\mathrm{H}(7 \mathrm{~A})$ & 2519 & 5675 & -1015 & 19 \\
\hline $\mathrm{H}(7 \mathrm{AB})$ & 348 & 6336 & -1390 & 19 \\
\hline $\mathrm{H}(8 \mathrm{~A})$ & 1922 & 8060 & 1118 & 17 \\
\hline $\mathrm{H}(8 \mathrm{AB})$ & 287 & 6460 & 653 & 17 \\
\hline $\mathrm{H}(9)$ & 3764 & 5726 & 1298 & 14 \\
\hline $\mathrm{H}(10 \mathrm{~A})$ & 2713 & 7975 & 3358 & 19 \\
\hline $\mathrm{H}(10 \mathrm{~B})$ & 2323 & 6295 & 3083 & 19 \\
\hline $\mathrm{H}(11 \mathrm{~A})$ & 9590 & 6026 & 5984 & 23 \\
\hline $\mathrm{H}(11 \mathrm{~B})$ & 10403 & 7460 & 5764 & 23 \\
\hline $\mathrm{H}(12 \mathrm{~A})$ & 9009 & 8261 & 7613 & 19 \\
\hline $\mathrm{H}(12 \mathrm{~B})$ & 6717 & 6855 & 6885 & 19 \\
\hline
\end{tabular}




\section{$\underline{\text { Crystal data for } \mathbf{1 1}}$}

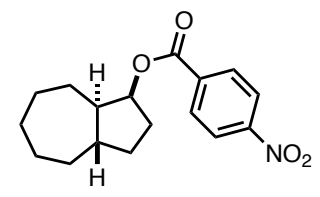

11

The crystal structure of $\mathbf{1 1}$ was solved in the triclinic space group $P \overline{1}$. The asymmetric unit contains three independent molecules of $\mathrm{C}_{17} \mathrm{H}_{21} \mathrm{NO}_{4}$ (11). Each perhydroazulen unit was found to be disordered to various degrees. The disorder was modeled with the help of same distance restraints on 1,2 and 1,3 distances, similarity restraints on the anisotropic displacement parameters ${ }^{5}$ and advanced rigid bond restraints ${ }^{6}$. The disorder ratios were allowed to refine freely and converged to 0.922(4) for the first, $0.613(3) / 0.266(3) / 0.120(2)$ for the second and to $0.613(3)$ for the third molecule.

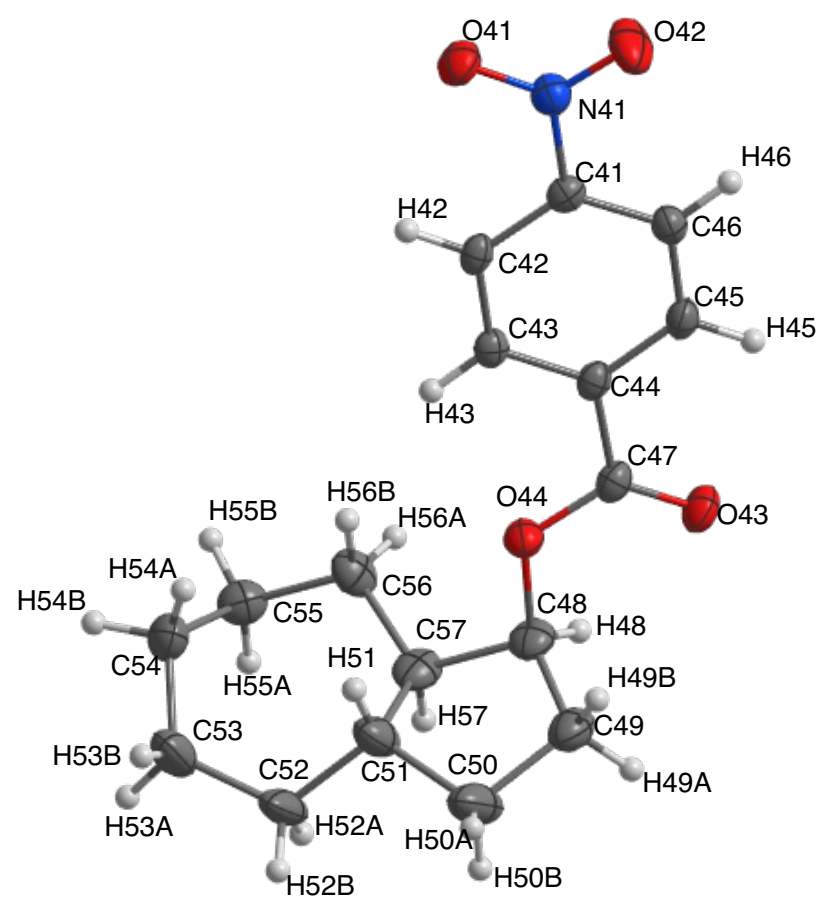

Thermal ellipsoid plot of $\mathrm{C}_{17} \mathrm{H}_{21} \mathrm{NO}_{4}$ (11) 
Table S6. Crystal data and structure refinement for 11.

Identification code

Empirical formula

Formula weight

Temperature

Wavelength

Crystal system

Space group

Unit cell dimensions

Volume

Z

Density (calculated)

Absorption coefficient

$\mathrm{F}(000)$

Crystal size

Theta range for data collection

Index ranges

Reflections collected

Independent reflections

Completeness to theta $=25.242^{\circ}$

Absorption correction

Refinement method

Data / restraints / parameters

Goodness-of-fit on $F^{2}$

Final R indices $[\mathrm{I}>2 \sigma(\mathrm{I})]$

$\mathrm{R}$ indices (all data)

Extinction coefficient

Largest diff. peak and hole
1978701

$\mathrm{C}_{17} \mathrm{H}_{21} \mathrm{~N} \mathrm{O}_{4}$

303.35

100(2) K

$0.71073 \AA$

Triclinic

$P \overline{1}$

$$
\begin{array}{ll}
\mathrm{a}=6.8690(3) \AA & \alpha=69.1744(14)^{\circ} . \\
\mathrm{b}=18.7339(8) \AA & \beta=87.7497(15)^{\circ} . \\
\mathrm{c}=19.4363(9) \AA & \gamma=81.4656(15)^{\circ} .
\end{array}
$$

\section{$2311.59(18) \AA^{3}$}

6

$1.307 \mathrm{Mg} / \mathrm{m}^{3}$

$0.093 \mathrm{~mm}^{-1}$

972

$0.905 \times 0.166 \times 0.153 \mathrm{~mm}^{3}$

1.306 to $26.022^{\circ}$.

$-8<=\mathrm{h}<=8,-23<=\mathrm{k}<=23,-23<=\mathrm{l}<=23$

110868

$9117[\mathrm{R}(\mathrm{int})=0.0790]$

$100.0 \%$

Semi-empirical from equivalents

Full-matrix least-squares on $F^{2}$

9117 / 2997 / 829

1.020

$\mathrm{R}_{1}=0.0486, \mathrm{wR}_{2}=0.1075$

$\mathrm{R}_{1}=0.0776, \mathrm{wR}_{2}=0.1218$

$0.0072(6)$

0.276 and -0.259 e. $\AA^{-3}$ 
Table S7. Atomic coordinates (x 10 $)$ and equivalent isotropic displacement parameters $\left(\AA^{2} \times 10^{3}\right)$ for 11. $U(e q)$ is defined as one third of the trace of the orthogonalized $U^{i j}$ tensor.

\begin{tabular}{|c|c|c|c|c|}
\hline & $\mathrm{x}$ & $\mathrm{y}$ & $\mathrm{z}$ & $\mathrm{U}(\mathrm{eq})$ \\
\hline $\mathrm{O}(1)$ & $8832(2)$ & $2998(1)$ & $10041(1)$ & $36(1)$ \\
\hline $\mathrm{O}(2)$ & $11130(2)$ & $3717(1)$ & $9675(1)$ & $36(1)$ \\
\hline $\mathrm{O}(3)$ & $1728(2)$ & 6191(1) & $8700(1)$ & $27(1)$ \\
\hline $\mathrm{O}(4)$ & $4161(2)$ & $6921(1)$ & $8514(1)$ & $27(1)$ \\
\hline $\mathrm{N}(1)$ & $9391(2)$ & $3627(1)$ & $9741(1)$ & $25(1)$ \\
\hline $\mathrm{C}(1)$ & $7881(2)$ & $4312(1)$ & $9454(1)$ & $21(1)$ \\
\hline$C(2)$ & $8453(2)$ & $5034(1)$ & $9255(1)$ & $21(1)$ \\
\hline$C(3)$ & $7022(2)$ & $5673(1)$ & $9004(1)$ & $22(1)$ \\
\hline$C(4)$ & $5060(2)$ & $5580(1)$ & $8952(1)$ & $20(1)$ \\
\hline$C(5)$ & $4546(3)$ & $4845(1)$ & $9142(1)$ & $23(1)$ \\
\hline$C(6)$ & $5960(2)$ & 4199(1) & $9401(1)$ & $23(1)$ \\
\hline$C(7)$ & $3458(3)$ & $6251(1)$ & $8708(1)$ & $22(1)$ \\
\hline$C(8)$ & 2701(3) & $7623(1)$ & $8267(1)$ & $27(1)$ \\
\hline$C(9)$ & $2144(3)$ & $7812(1)$ & $7462(1)$ & $31(1)$ \\
\hline$C(10)$ & $3345(3)$ & $8452(1)$ & $7016(1)$ & $35(1)$ \\
\hline $\mathrm{C}(11)$ & $4876(3)$ & $8489(1)$ & $7548(1)$ & $28(1)$ \\
\hline$C(12)$ & $5615(3)$ & $9267(1)$ & $7307(1)$ & $34(1)$ \\
\hline$C(13)$ & $7537(3)$ & $9289(1)$ & $7663(1)$ & $39(1)$ \\
\hline$C(14)$ & $7852(3)$ & $8789(1)$ & $8474(1)$ & $38(1)$ \\
\hline$C(15)$ & $6059(3)$ & $8773(1)$ & $8968(1)$ & $37(1)$ \\
\hline$C(15 A)$ & $7105(18)$ & $8026(8)$ & $8795(11)$ & $32(5)$ \\
\hline$C(16)$ & $4858(3)$ & $8132(1)$ & $8990(1)$ & $35(1)$ \\
\hline $\mathrm{C}(17)$ & $3746(3)$ & $8280(1)$ & $8275(1)$ & $27(1)$ \\
\hline $\mathrm{O}(21)$ & $6750(2)$ & $6966(1)$ & $6779(1)$ & $42(1)$ \\
\hline $\mathrm{O}(22)$ & $4514(2)$ & $6216(1)$ & $7059(1)$ & $32(1)$ \\
\hline $\mathrm{O}(23)$ & $13977(2)$ & $3795(1)$ & $8038(1)$ & $38(1)$ \\
\hline $\mathrm{N}(21)$ & $6235(2)$ & $6326(1)$ & $7022(1)$ & $27(1)$ \\
\hline $\mathrm{C}(21)$ & $7774(2)$ & $5647(1)$ & $7285(1)$ & $23(1)$ \\
\hline$C(22)$ & $9695(3)$ & $5767(1)$ & $7340(1)$ & $24(1)$ \\
\hline $\mathrm{C}(23)$ & $11129(3)$ & $5128(1)$ & $7576(1)$ & $25(1)$ \\
\hline$C(24)$ & $10649(3)$ & $4389(1)$ & $7747(1)$ & $24(1)$ \\
\hline$C(25)$ & 8699(3) & $4287(1)$ & $7698(1)$ & $24(1)$ \\
\hline$C(26)$ & $7239(3)$ & $4921(1)$ & $7464(1)$ & $23(1)$ \\
\hline $\mathrm{C}(27)$ & $12288(3)$ & $3727(1)$ & $7973(1)$ & $28(1)$ \\
\hline $\mathrm{O}(24)$ & $11650(30)$ & $3046(8)$ & $8057(12)$ & $34(3)$ \\
\hline
\end{tabular}




\begin{tabular}{|c|c|c|c|c|}
\hline $\mathrm{C}(28)$ & $13083(16)$ & $2335(6)$ & $8307(6)$ & $33(2)$ \\
\hline$C(29)$ & 13979(14) & $2145(6)$ & $7639(6)$ & $37(2)$ \\
\hline$C(30)$ & $13066(12)$ & $1450(4)$ & $7632(5)$ & $37(2)$ \\
\hline$C(31)$ & 11241(13) & $1420(6)$ & $8111(6)$ & $32(1)$ \\
\hline$C(32)$ & $10558(18)$ & $616(7)$ & $8407(9)$ & $47(1)$ \\
\hline$C(33)$ & $8488(17)$ & $622(6)$ & $8717(6)$ & $65(3)$ \\
\hline C(34) & $7889(19)$ & $1029(8)$ & $9246(8)$ & $66(1)$ \\
\hline$C(35)$ & $9458(6)$ & $1154(2)$ & $9704(2)$ & $50(1)$ \\
\hline$C(36)$ & $10455(12)$ & $1847(7)$ & $9252(6)$ & $44(1)$ \\
\hline$C(37)$ & $11955(13)$ & $1671(6)$ & $8709(5)$ & $30(2)$ \\
\hline $\mathrm{O}(24 \mathrm{~A})$ & $11580(70)$ & $3054(19)$ & $8150(30)$ & $30(3)$ \\
\hline $\mathrm{C}(28 \mathrm{~A})$ & $13180(40)$ & $2393(15)$ & $8411(14)$ & $38(4)$ \\
\hline$C(29 A)$ & $14210(40)$ & $2258(15)$ & $7739(14)$ & $50(5)$ \\
\hline$C(30 A)$ & $13440(30)$ & $1580(14)$ & $7657(13)$ & $55(4)$ \\
\hline$C(31 \mathrm{~A})$ & $11590(30)$ & $1480(15)$ & $8116(14)$ & $39(3)$ \\
\hline $\mathrm{C}(32 \mathrm{~A})$ & $10740(40)$ & $709(16)$ & $8350(20)$ & $47(1)$ \\
\hline$C(33 A)$ & $8540(30)$ & $754(13)$ & $8541(12)$ & $42(3)$ \\
\hline$C(34 A)$ & $7940(50)$ & $994(18)$ & $9184(18)$ & $66(1)$ \\
\hline$C(35 A)$ & $8457(12)$ & $1806(5)$ & $9092(4)$ & $42(2)$ \\
\hline$C(36 A)$ & $10580(30)$ & $1758(19)$ & $9319(15)$ & $44(1)$ \\
\hline$C(37 A)$ & $12160(30)$ & $1684(15)$ & $8768(13)$ & $35(3)$ \\
\hline $\mathrm{O}(24 \mathrm{~B})$ & $11840(90)$ & $3112(17)$ & $8120(40)$ & $34(6)$ \\
\hline $\mathrm{C}(28 \mathrm{~B})$ & $13390(50)$ & $2435(15)$ & $8425(12)$ & $41(4)$ \\
\hline$C(29 B)$ & $13440(30)$ & $2208(10)$ & $9264(9)$ & $54(4)$ \\
\hline$C(30 B)$ & $12110(30)$ & $1586(11)$ & $9570(8)$ & $63(5)$ \\
\hline$C(31 B)$ & 10940(30) & $1611(10)$ & $8894(9)$ & $45(4)$ \\
\hline $\mathrm{C}(32 \mathrm{~B})$ & $10120(30)$ & $825(10)$ & 9034(9) & $59(4)$ \\
\hline$C(33 B)$ & $9530(30)$ & $728(14)$ & $8322(11)$ & $47(1)$ \\
\hline $\mathrm{C}(34 \mathrm{~B})$ & $11160(30)$ & $553(10)$ & $7850(10)$ & $52(4)$ \\
\hline$C(35 B)$ & $12020(30)$ & $1231(9)$ & $7307(9)$ & $51(4)$ \\
\hline$C(36 B)$ & $11900(30)$ & 1939(9) & $7531(8)$ & $43(4)$ \\
\hline$C(37 B)$ & $12500(30)$ & $1782(10)$ & $8310(8)$ & $42(3)$ \\
\hline $\mathrm{O}(41)$ & $-2263(2)$ & $3671(1)$ & $6283(1)$ & $34(1)$ \\
\hline $\mathrm{O}(42)$ & $500(2)$ & $2958(1)$ & $6285(1)$ & $51(1)$ \\
\hline $\mathrm{O}(43)$ & $5349(2)$ & $6139(1)$ & $5484(1)$ & $32(1)$ \\
\hline $\mathrm{O}(44)$ & $2358(2)$ & $6864(1)$ & $5249(1)$ & $28(1)$ \\
\hline $\mathrm{N}(41)$ & $-480(2)$ & $3582(1)$ & $6204(1)$ & $28(1)$ \\
\hline$C(41)$ & $549(2)$ & $4259(1)$ & $6014(1)$ & $21(1)$ \\
\hline$C(42)$ & $-557(2)$ & $4981(1)$ & $5864(1)$ & $21(1)$ \\
\hline$C(43)$ & $415(2)$ & $5615(1)$ & $5679(1)$ & $21(1)$ \\
\hline
\end{tabular}




$\begin{array}{lclll}\mathrm{C}(44) & 2460(2) & 5527(1) & 5656(1) & 21(1) \\ \mathrm{C}(45) & 3529(3) & 4794(1) & 5810(1) & 25(1) \\ \mathrm{C}(46) & 2579(3) & 4152(1) & 5988(1) & 24(1) \\ \mathrm{C}(47) & 3575(3) & 6197(1) & 5459(1) & 24(1) \\ \mathrm{C}(48) & 3265(3) & 7570(1) & 5048(1) & 32(1) \\ \mathrm{C}(49) & 3960(3) & 7796(1) & 4250(1) & 38(1) \\ \mathrm{C}(50) & 2569(4) & 8520(1) & 3807(1) & 55(1) \\ \mathrm{C}(51) & 737(3) & 8523(1) & 4283(1) & 40(1) \\ \mathrm{C}(52) & -322(10) & 9347(3) & 4137(4) & 59(2) \\ \mathrm{C}(53) & -2401(13) & 9414(6) & 4431(4) & 54(3) \\ \mathrm{C}(54) & -2814(5) & 8840(2) & 5181(2) & 38(1) \\ \mathrm{C}(55) & -1235(17) & 8595(7) & 5782(4) & 33(2) \\ \mathrm{C}(56) & 318(15) & 7948(5) & 5725(5) & 34(2) \\ \mathrm{C}(52 \mathrm{~A}) & -842(18) & 9238(7) & 4000(6) & 50(3) \\ \mathrm{C}(53 \mathrm{~A}) & -2640(20) & 9246(11) & 4475(7) & 45(3) \\ \mathrm{C}(54 \mathrm{~A}) & -2279(11) & 9359(4) & 5188(3) & 51(2) \\ \mathrm{C}(55 \mathrm{~A}) & -1220(30) & 8680(13) & 5818(8) & 42(4) \\ \mathrm{C}(56 \mathrm{~A}) & -30(30) & 8016(10) & 5649(9) & 35(3) \\ \mathrm{C}(57) & 1623(3) & 8211(1) & 5061(1) & 34(1) \\ & & & & \end{array}$


Table S8. Bond lengths $[\AA]$ and angles $\left[{ }^{\circ}\right]$ for $\mathbf{1 1}$.

\begin{tabular}{|c|c|c|c|}
\hline $\mathrm{O}(1)-\mathrm{N}(1)$ & $1.2270(19)$ & $\mathrm{C}(14)-\mathrm{H}(14 \mathrm{~B})$ & 0.9900 \\
\hline $\mathrm{O}(2)-\mathrm{N}(1)$ & $1.2265(19)$ & $\mathrm{C}(14)-\mathrm{H}(14 \mathrm{C})$ & 0.9900 \\
\hline $\mathrm{O}(3)-\mathrm{C}(7)$ & $1.211(2)$ & $\mathrm{C}(14)-\mathrm{H}(14 \mathrm{D})$ & 0.9900 \\
\hline $\mathrm{O}(4)-\mathrm{C}(7)$ & $1.333(2)$ & $C(15)-C(16)$ & $1.542(3)$ \\
\hline $\mathrm{O}(4)-\mathrm{C}(8)$ & $1.472(2)$ & $\mathrm{C}(15)-\mathrm{H}(15 \mathrm{~A})$ & 0.9900 \\
\hline $\mathrm{N}(1)-\mathrm{C}(1)$ & $1.474(2)$ & $\mathrm{C}(15)-\mathrm{H}(15 \mathrm{~B})$ & 0.9900 \\
\hline$C(1)-C(6)$ & $1.381(2)$ & $C(15 A)-C(16)$ & $1.576(13)$ \\
\hline$C(1)-C(2)$ & $1.382(2)$ & $\mathrm{C}(15 \mathrm{~A})-\mathrm{H}(15 \mathrm{C})$ & 0.9900 \\
\hline $\mathrm{C}(2)-\mathrm{C}(3)$ & $1.382(2)$ & $\mathrm{C}(15 \mathrm{~A})-\mathrm{H}(15 \mathrm{D})$ & 0.9900 \\
\hline $\mathrm{C}(2)-\mathrm{H}(2)$ & 0.9500 & $C(16)-C(17)$ & $1.528(3)$ \\
\hline$C(3)-C(4)$ & $1.396(2)$ & $\mathrm{C}(16)-\mathrm{H}(16 \mathrm{~A})$ & 0.9900 \\
\hline $\mathrm{C}(3)-\mathrm{H}(3)$ & 0.9500 & $\mathrm{C}(16)-\mathrm{H}(16 \mathrm{~B})$ & 0.9900 \\
\hline$C(4)-C(5)$ & $1.389(2)$ & $\mathrm{C}(16)-\mathrm{H}(16 \mathrm{C})$ & 0.9900 \\
\hline$C(4)-C(7)$ & $1.491(2)$ & $\mathrm{C}(16)-\mathrm{H}(16 \mathrm{D})$ & 0.9900 \\
\hline$C(5)-C(6)$ & $1.385(2)$ & $\mathrm{C}(17)-\mathrm{H}(17)$ & 1.0000 \\
\hline $\mathrm{C}(5)-\mathrm{H}(5)$ & 0.9500 & $\mathrm{O}(21)-\mathrm{N}(21)$ & $1.224(2)$ \\
\hline $\mathrm{C}(6)-\mathrm{H}(6)$ & 0.9500 & $\mathrm{O}(22)-\mathrm{N}(21)$ & $1.2246(19)$ \\
\hline$C(8)-C(17)$ & $1.519(3)$ & $\mathrm{O}(23)-\mathrm{C}(27)$ & $1.202(2)$ \\
\hline C(8)-C(9) & $1.527(3)$ & $\mathrm{N}(21)-\mathrm{C}(21)$ & $1.475(2)$ \\
\hline $\mathrm{C}(8)-\mathrm{H}(8)$ & 1.0000 & $\mathrm{C}(21)-\mathrm{C}(26)$ & $1.382(2)$ \\
\hline $\mathrm{C}(9)-\mathrm{C}(10)$ & $1.539(3)$ & $\mathrm{C}(21)-\mathrm{C}(22)$ & $1.385(2)$ \\
\hline $\mathrm{C}(9)-\mathrm{H}(9 \mathrm{~A})$ & 0.9900 & $\mathrm{C}(22)-\mathrm{C}(23)$ & $1.381(2)$ \\
\hline $\mathrm{C}(9)-\mathrm{H}(9 \mathrm{AB})$ & 0.9900 & $\mathrm{C}(22)-\mathrm{H}(22)$ & 0.9500 \\
\hline $\mathrm{C}(10)-\mathrm{C}(11)$ & $1.528(3)$ & $\mathrm{C}(23)-\mathrm{C}(24)$ & $1.390(3)$ \\
\hline $\mathrm{C}(10)-\mathrm{H}(10 \mathrm{~A})$ & 0.9900 & $\mathrm{C}(23)-\mathrm{H}(23)$ & 0.9500 \\
\hline $\mathrm{C}(10)-\mathrm{H}(10 \mathrm{~B})$ & 0.9900 & $\mathrm{C}(24)-\mathrm{C}(25)$ & $1.393(2)$ \\
\hline$C(11)-C(12)$ & $1.523(3)$ & $\mathrm{C}(24)-\mathrm{C}(27)$ & $1.495(3)$ \\
\hline$C(11)-C(17)$ & $1.538(3)$ & $C(25)-C(26)$ & $1.386(2)$ \\
\hline $\mathrm{C}(11)-\mathrm{H}(11)$ & 1.0000 & $\mathrm{C}(25)-\mathrm{H}(25)$ & 0.9500 \\
\hline$C(12)-C(13)$ & $1.527(3)$ & $\mathrm{C}(26)-\mathrm{H}(26)$ & 0.9500 \\
\hline $\mathrm{C}(12)-\mathrm{H}(12 \mathrm{~A})$ & 0.9900 & $\mathrm{C}(27)-\mathrm{O}(24 \mathrm{~B})$ & $1.17(4)$ \\
\hline $\mathrm{C}(12)-\mathrm{H}(12 \mathrm{~B})$ & 0.9900 & $\mathrm{C}(27)-\mathrm{O}(24 \mathrm{~A})$ & $1.34(5)$ \\
\hline$C(13)-C(14)$ & $1.526(3)$ & $\mathrm{C}(27)-\mathrm{O}(24)$ & $1.36(2)$ \\
\hline $\mathrm{C}(13)-\mathrm{H}(13 \mathrm{~A})$ & 0.9900 & $\mathrm{O}(24)-\mathrm{C}(28)$ & $1.472(7)$ \\
\hline $\mathrm{C}(13)-\mathrm{H}(13 \mathrm{~B})$ & 0.9900 & $\mathrm{C}(28)-\mathrm{C}(37)$ & $1.519(7)$ \\
\hline$C(14)-C(15 A)$ & $1.502(12)$ & $\mathrm{C}(28)-\mathrm{C}(29)$ & $1.547(7)$ \\
\hline$C(14)-C(15)$ & $1.529(3)$ & $\mathrm{C}(28)-\mathrm{H}(28)$ & 1.0000 \\
\hline $\mathrm{C}(14)-\mathrm{H}(14 \mathrm{~A})$ & 0.9900 & $C(29)-C(30)$ & $1.532(6)$ \\
\hline
\end{tabular}




\begin{tabular}{|c|c|c|c|}
\hline C(29)-H(29A) & 0.9900 & $C(33 A)-C(34 A)$ & $1.496(14)$ \\
\hline C(29)-H(29B) & 0.9900 & $\mathrm{C}(33 \mathrm{~A})-\mathrm{H}(33 \mathrm{C})$ & 0.9900 \\
\hline$C(30)-C(31)$ & $1.527(6)$ & C(33A)-H(33D) & 0.9900 \\
\hline C(30)-H(30A) & 0.9900 & $\mathrm{C}(34 \mathrm{~A})-\mathrm{C}(35 \mathrm{~A})$ & $1.559(18)$ \\
\hline $\mathrm{C}(30)-\mathrm{H}(30 \mathrm{~B})$ & 0.9900 & $\mathrm{C}(34 \mathrm{~A})-\mathrm{H}(34 \mathrm{C})$ & 0.9900 \\
\hline$C(31)-C(37)$ & $1.523(7)$ & $\mathrm{C}(34 \mathrm{~A})-\mathrm{H}(34 \mathrm{D})$ & 0.9900 \\
\hline $\mathrm{C}(31)-\mathrm{C}(32)$ & $1.546(7)$ & $C(35 A)-C(36 A)$ & $1.522(17)$ \\
\hline $\mathrm{C}(31)-\mathrm{H}(31)$ & 1.0000 & $\mathrm{C}(35 \mathrm{~A})-\mathrm{H}(35 \mathrm{C})$ & 0.9900 \\
\hline$C(32)-C(33)$ & $1.522(7)$ & $\mathrm{C}(35 \mathrm{~A})-\mathrm{H}(35 \mathrm{D})$ & 0.9900 \\
\hline C(32)-H(32A) & 0.9900 & $C(36 A)-C(37 A)$ & $1.519(13)$ \\
\hline $\mathrm{C}(32)-\mathrm{H}(32 \mathrm{~B})$ & 0.9900 & $\mathrm{C}(36 \mathrm{~A})-\mathrm{H}(36 \mathrm{C})$ & 0.9900 \\
\hline C(33)-C(34) & $1.495(8)$ & $\mathrm{C}(36 \mathrm{~A})-\mathrm{H}(36 \mathrm{D})$ & 0.9900 \\
\hline C(33)-H(33A) & 0.9900 & $\mathrm{C}(37 \mathrm{~A})-\mathrm{H}(37 \mathrm{~A})$ & 1.0000 \\
\hline C(33)-H(33B) & 0.9900 & $\mathrm{O}(24 \mathrm{~B})-\mathrm{C}(28 \mathrm{~B})$ & $1.487(16)$ \\
\hline$C(34)-C(35)$ & $1.520(12)$ & $\mathrm{C}(28 \mathrm{~B})-\mathrm{C}(37 \mathrm{~B})$ & $1.532(15)$ \\
\hline $\mathrm{C}(34)-\mathrm{H}(34 \mathrm{~A})$ & 0.9900 & $\mathrm{C}(28 \mathrm{~B})-\mathrm{C}(29 \mathrm{~B})$ & $1.533(18)$ \\
\hline C(34)-H(34B) & 0.9900 & $\mathrm{C}(28 \mathrm{~B})-\mathrm{H}(28 \mathrm{~B})$ & 1.0000 \\
\hline$C(35)-C(36)$ & $1.527(11)$ & $C(29 B)-C(30 B)$ & $1.525(16)$ \\
\hline $\mathrm{C}(35)-\mathrm{H}(35 \mathrm{~A})$ & 0.9900 & C(29B)-H(29E) & 0.9900 \\
\hline $\mathrm{C}(35)-\mathrm{H}(35 \mathrm{~B})$ & 0.9900 & $\mathrm{C}(29 B)-\mathrm{H}(29 \mathrm{~F})$ & 0.9900 \\
\hline$C(36)-C(37)$ & $1.532(7)$ & $\mathrm{C}(30 \mathrm{~B})-\mathrm{C}(31 \mathrm{~B})$ & $1.551(15)$ \\
\hline $\mathrm{C}(36)-\mathrm{H}(36 \mathrm{~A})$ & 0.9900 & $\mathrm{C}(30 \mathrm{~B})-\mathrm{H}(30 \mathrm{E})$ & 0.9900 \\
\hline $\mathrm{C}(36)-\mathrm{H}(36 \mathrm{~B})$ & 0.9900 & $\mathrm{C}(30 \mathrm{~B})-\mathrm{H}(30 \mathrm{~F})$ & 0.9900 \\
\hline $\mathrm{C}(37)-\mathrm{H}(37)$ & 1.0000 & $\mathrm{C}(31 \mathrm{~B})-\mathrm{C}(37 \mathrm{~B})$ & $1.514(15)$ \\
\hline $\mathrm{O}(24 \mathrm{~A})-\mathrm{C}(28 \mathrm{~A})$ & $1.478(15)$ & $C(31 B)-C(32 B)$ & $1.583(15)$ \\
\hline $\mathrm{C}(28 \mathrm{~A})-\mathrm{C}(37 \mathrm{~A})$ & $1.524(14)$ & $\mathrm{C}(31 \mathrm{~B})-\mathrm{H}(31 \mathrm{~B})$ & 1.0000 \\
\hline$C(28 \mathrm{~A})-\mathrm{C}(29 \mathrm{~A})$ & $1.543(15)$ & $\mathrm{C}(32 \mathrm{~B})-\mathrm{C}(33 \mathrm{~B})$ & $1.534(15)$ \\
\hline $\mathrm{C}(28 \mathrm{~A})-\mathrm{H}(28 \mathrm{~A})$ & 1.0000 & $\mathrm{C}(32 \mathrm{~B})-\mathrm{H}(32 \mathrm{E})$ & 0.9900 \\
\hline$C(29 A)-C(30 A)$ & $1.508(13)$ & $\mathrm{C}(32 \mathrm{~B})-\mathrm{H}(32 \mathrm{~F})$ & 0.9900 \\
\hline $\mathrm{C}(29 \mathrm{~A})-\mathrm{H}(29 \mathrm{C})$ & 0.9900 & $\mathrm{C}(33 \mathrm{~B})-\mathrm{C}(34 \mathrm{~B})$ & $1.491(16)$ \\
\hline$C(29 A)-H(29 D)$ & 0.9900 & $\mathrm{C}(33 \mathrm{~B})-\mathrm{H}(33 \mathrm{E})$ & 0.9900 \\
\hline$C(30 A)-C(31 A)$ & $1.520(14)$ & $\mathrm{C}(33 \mathrm{~B})-\mathrm{H}(33 \mathrm{~F})$ & 0.9900 \\
\hline $\mathrm{C}(30 \mathrm{~A})-\mathrm{H}(30 \mathrm{C})$ & 0.9900 & $\mathrm{C}(34 \mathrm{~B})-\mathrm{C}(35 \mathrm{~B})$ & $1.515(16)$ \\
\hline $\mathrm{C}(30 \mathrm{~A})-\mathrm{H}(30 \mathrm{D})$ & 0.9900 & $\mathrm{C}(34 \mathrm{~B})-\mathrm{H}(34 \mathrm{E})$ & 0.9900 \\
\hline$C(31 \mathrm{~A})-\mathrm{C}(37 \mathrm{~A})$ & $1.528(14)$ & $\mathrm{C}(34 \mathrm{~B})-\mathrm{H}(34 \mathrm{~F})$ & 0.9900 \\
\hline$C(31 \mathrm{~A})-\mathrm{C}(32 \mathrm{~A})$ & $1.546(13)$ & $C(35 B)-C(36 B)$ & $1.528(15)$ \\
\hline $\mathrm{C}(31 \mathrm{~A})-\mathrm{H}(31 \mathrm{~A})$ & 1.0000 & $\mathrm{C}(35 \mathrm{~B})-\mathrm{H}(35 \mathrm{E})$ & 0.9900 \\
\hline$C(32 A)-C(33 A)$ & $1.540(14)$ & $\mathrm{C}(35 \mathrm{~B})-\mathrm{H}(35 \mathrm{~F})$ & 0.9900 \\
\hline $\mathrm{C}(32 \mathrm{~A})-\mathrm{H}(32 \mathrm{C})$ & 0.9900 & $C(36 B)-C(37 B)$ & $1.498(14)$ \\
\hline $\mathrm{C}(32 \mathrm{~A})-\mathrm{H}(32 \mathrm{D})$ & 0.9900 & $\mathrm{C}(36 \mathrm{~B})-\mathrm{H}(36 \mathrm{E})$ & 0.9900 \\
\hline
\end{tabular}




\begin{tabular}{|c|c|c|c|}
\hline $\mathrm{C}(36 \mathrm{~B})-\mathrm{H}(36 \mathrm{~F})$ & 0.9900 & $\mathrm{C}(54)-\mathrm{H}(54 \mathrm{~A})$ & 0.9900 \\
\hline $\mathrm{C}(37 \mathrm{~B})-\mathrm{H}(37 \mathrm{~B})$ & 1.0000 & $\mathrm{C}(54)-\mathrm{H}(54 \mathrm{~B})$ & 0.9900 \\
\hline $\mathrm{O}(41)-\mathrm{N}(41)$ & $1.2224(19)$ & $\mathrm{C}(55)-\mathrm{C}(56)$ & $1.524(8)$ \\
\hline $\mathrm{O}(42)-\mathrm{N}(41)$ & $1.2196(19)$ & $\mathrm{C}(55)-\mathrm{H}(55 \mathrm{~A})$ & 0.9900 \\
\hline $\mathrm{O}(43)-\mathrm{C}(47)$ & $1.209(2)$ & $\mathrm{C}(55)-\mathrm{H}(55 \mathrm{~B})$ & 0.9900 \\
\hline $\mathrm{O}(44)-\mathrm{C}(47)$ & $1.334(2)$ & $C(56)-C(57)$ & $1.518(7)$ \\
\hline $\mathrm{O}(44)-\mathrm{C}(48)$ & $1.467(2)$ & $\mathrm{C}(56)-\mathrm{H}(56 \mathrm{~A})$ & 0.9900 \\
\hline $\mathrm{N}(41)-\mathrm{C}(41)$ & $1.468(2)$ & $\mathrm{C}(56)-\mathrm{H}(56 \mathrm{~B})$ & 0.9900 \\
\hline$C(41)-C(46)$ & $1.380(2)$ & $\mathrm{C}(52 \mathrm{~A})-\mathrm{C}(53 \mathrm{~A})$ & $1.512(12)$ \\
\hline$C(41)-C(42)$ & $1.386(2)$ & $\mathrm{C}(52 \mathrm{~A})-\mathrm{H}(52 \mathrm{C})$ & 0.9900 \\
\hline$C(42)-C(43)$ & $1.379(2)$ & $\mathrm{C}(52 \mathrm{~A})-\mathrm{H}(52 \mathrm{D})$ & 0.9900 \\
\hline $\mathrm{C}(42)-\mathrm{H}(42)$ & 0.9500 & $C(53 \mathrm{~A})-\mathrm{C}(54 \mathrm{~A})$ & $1.511(13)$ \\
\hline$C(43)-C(44)$ & $1.390(2)$ & $\mathrm{C}(53 \mathrm{~A})-\mathrm{H}(53 \mathrm{C})$ & 0.9900 \\
\hline $\mathrm{C}(43)-\mathrm{H}(43)$ & 0.9500 & $\mathrm{C}(53 \mathrm{~A})-\mathrm{H}(53 \mathrm{D})$ & 0.9900 \\
\hline$C(44)-C(45)$ & $1.392(2)$ & $\mathrm{C}(54 \mathrm{~A})-\mathrm{C}(55 \mathrm{~A})$ & $1.527(13)$ \\
\hline$C(44)-C(47)$ & $1.493(2)$ & $\mathrm{C}(54 \mathrm{~A})-\mathrm{H}(54 \mathrm{C})$ & 0.9900 \\
\hline$C(45)-C(46)$ & $1.383(3)$ & $\mathrm{C}(54 \mathrm{~A})-\mathrm{H}(54 \mathrm{D})$ & 0.9900 \\
\hline $\mathrm{C}(45)-\mathrm{H}(45)$ & 0.9500 & $C(55 A)-C(56 A)$ & $1.514(13)$ \\
\hline $\mathrm{C}(46)-\mathrm{H}(46)$ & 0.9500 & $\mathrm{C}(55 \mathrm{~A})-\mathrm{H}(55 \mathrm{C})$ & 0.9900 \\
\hline $\mathrm{C}(48)-\mathrm{C}(57)$ & $1.527(3)$ & $\mathrm{C}(55 \mathrm{~A})-\mathrm{H}(55 \mathrm{D})$ & 0.9900 \\
\hline $\mathrm{C}(48)-\mathrm{C}(49)$ & $1.532(3)$ & $C(56 A)-C(57)$ & $1.567(11)$ \\
\hline $\mathrm{C}(48)-\mathrm{H}(48)$ & 1.0000 & $\mathrm{C}(56 \mathrm{~A})-\mathrm{H}(56 \mathrm{C})$ & 0.9900 \\
\hline$C(49)-C(50)$ & $1.531(3)$ & $\mathrm{C}(56 \mathrm{~A})-\mathrm{H}(56 \mathrm{D})$ & 0.9900 \\
\hline $\mathrm{C}(49)-\mathrm{H}(49 \mathrm{~A})$ & 0.9900 & $\mathrm{C}(57)-\mathrm{H}(57)$ & 1.0000 \\
\hline C(49)-H(49B) & 0.9900 & $\mathrm{C}(57)-\mathrm{H}(57 \mathrm{~A})$ & 1.0000 \\
\hline $\mathrm{C}(50)-\mathrm{C}(51)$ & $1.533(3)$ & & \\
\hline $\mathrm{C}(50)-\mathrm{H}(50 \mathrm{~A})$ & 0.9900 & $\mathrm{C}(7)-\mathrm{O}(4)-\mathrm{C}(8)$ & $116.31(13)$ \\
\hline $\mathrm{C}(50)-\mathrm{H}(50 \mathrm{~B})$ & 0.9900 & $\mathrm{O}(2)-\mathrm{N}(1)-\mathrm{O}(1)$ & $123.54(15)$ \\
\hline$C(51)-C(57)$ & $1.528(3)$ & $\mathrm{O}(2)-\mathrm{N}(1)-\mathrm{C}(1)$ & $118.54(15)$ \\
\hline$C(51)-C(52)$ & $1.536(6)$ & $\mathrm{O}(1)-\mathrm{N}(1)-\mathrm{C}(1)$ & $117.91(14)$ \\
\hline$C(51)-C(52 A)$ & $1.538(10)$ & $C(6)-C(1)-C(2)$ & $123.16(16)$ \\
\hline $\mathrm{C}(51)-\mathrm{H}(51)$ & 1.0000 & $\mathrm{C}(6)-\mathrm{C}(1)-\mathrm{N}(1)$ & $118.29(15)$ \\
\hline $\mathrm{C}(51)-\mathrm{H}(51 \mathrm{~A})$ & 1.0000 & $\mathrm{C}(2)-\mathrm{C}(1)-\mathrm{N}(1)$ & $118.55(15)$ \\
\hline$C(52)-C(53)$ & $1.522(7)$ & $\mathrm{C}(1)-\mathrm{C}(2)-\mathrm{C}(3)$ & $118.24(16)$ \\
\hline $\mathrm{C}(52)-\mathrm{H}(52 \mathrm{~A})$ & 0.9900 & $\mathrm{C}(1)-\mathrm{C}(2)-\mathrm{H}(2)$ & 120.9 \\
\hline $\mathrm{C}(52)-\mathrm{H}(52 \mathrm{~B})$ & 0.9900 & $\mathrm{C}(3)-\mathrm{C}(2)-\mathrm{H}(2)$ & 120.9 \\
\hline$C(53)-C(54)$ & $1.519(8)$ & $C(2)-C(3)-C(4)$ & $120.11(16)$ \\
\hline $\mathrm{C}(53)-\mathrm{H}(53 \mathrm{~A})$ & 0.9900 & $\mathrm{C}(2)-\mathrm{C}(3)-\mathrm{H}(3)$ & 119.9 \\
\hline $\mathrm{C}(53)-\mathrm{H}(53 \mathrm{~B})$ & 0.9900 & $\mathrm{C}(4)-\mathrm{C}(3)-\mathrm{H}(3)$ & 119.9 \\
\hline$C(54)-C(55)$ & $1.524(9)$ & $C(5)-C(4)-C(3)$ & $120.06(16)$ \\
\hline
\end{tabular}




\begin{tabular}{|c|c|}
\hline $\mathrm{C}(5)-\mathrm{C}(4)-\mathrm{C}(7)$ & $117.95(15)$ \\
\hline$C(3)-C(4)-C(7)$ & $121.98(16)$ \\
\hline$C(6)-C(5)-C(4)$ & $120.52(16)$ \\
\hline $\mathrm{C}(6)-\mathrm{C}(5)-\mathrm{H}(5)$ & 119.7 \\
\hline $\mathrm{C}(4)-\mathrm{C}(5)-\mathrm{H}(5)$ & 119.7 \\
\hline$C(1)-C(6)-C(5)$ & $117.89(16)$ \\
\hline $\mathrm{C}(1)-\mathrm{C}(6)-\mathrm{H}(6)$ & 121.1 \\
\hline $\mathrm{C}(5)-\mathrm{C}(6)-\mathrm{H}(6)$ & 121.1 \\
\hline $\mathrm{O}(3)-\mathrm{C}(7)-\mathrm{O}(4)$ & $124.43(16)$ \\
\hline $\mathrm{O}(3)-\mathrm{C}(7)-\mathrm{C}(4)$ & $123.82(16)$ \\
\hline $\mathrm{O}(4)-\mathrm{C}(7)-\mathrm{C}(4)$ & $111.74(14)$ \\
\hline $\mathrm{O}(4)-\mathrm{C}(8)-\mathrm{C}(17)$ & $106.42(14)$ \\
\hline $\mathrm{O}(4)-\mathrm{C}(8)-\mathrm{C}(9)$ & $109.44(14)$ \\
\hline C(17)-C(8)-C(9) & $105.56(15)$ \\
\hline $\mathrm{O}(4)-\mathrm{C}(8)-\mathrm{H}(8)$ & 111.7 \\
\hline $\mathrm{C}(17)-\mathrm{C}(8)-\mathrm{H}(8)$ & 111.7 \\
\hline $\mathrm{C}(9)-\mathrm{C}(8)-\mathrm{H}(8)$ & 111.7 \\
\hline C(8)-C(9)-C(10) & $105.47(15)$ \\
\hline $\mathrm{C}(8)-\mathrm{C}(9)-\mathrm{H}(9 \mathrm{~A})$ & 110.6 \\
\hline $\mathrm{C}(10)-\mathrm{C}(9)-\mathrm{H}(9 \mathrm{~A})$ & 110.6 \\
\hline C(8)-C(9)-H(9AB) & 110.6 \\
\hline $\mathrm{C}(10)-\mathrm{C}(9)-\mathrm{H}(9 \mathrm{AB})$ & 110.6 \\
\hline $\mathrm{H}(9 \mathrm{~A})-\mathrm{C}(9)-\mathrm{H}(9 \mathrm{AB})$ & 108.8 \\
\hline$C(11)-C(10)-C(9)$ & $106.43(15)$ \\
\hline$C(11)-C(10)-H(10 A)$ & 110.4 \\
\hline $\mathrm{C}(9)-\mathrm{C}(10)-\mathrm{H}(10 \mathrm{~A})$ & 110.4 \\
\hline$C(11)-C(10)-H(10 B)$ & 110.4 \\
\hline $\mathrm{C}(9)-\mathrm{C}(10)-\mathrm{H}(10 \mathrm{~B})$ & 110.4 \\
\hline $\mathrm{H}(10 \mathrm{~A})-\mathrm{C}(10)-\mathrm{H}(10 \mathrm{~B})$ & 108.6 \\
\hline $\mathrm{C}(12)-\mathrm{C}(11)-\mathrm{C}(10)$ & $112.96(15)$ \\
\hline $\mathrm{C}(12)-\mathrm{C}(11)-\mathrm{C}(17)$ & $114.78(16)$ \\
\hline $\mathrm{C}(10)-\mathrm{C}(11)-\mathrm{C}(17)$ & $101.48(15)$ \\
\hline $\mathrm{C}(12)-\mathrm{C}(11)-\mathrm{H}(11)$ & 109.1 \\
\hline $\mathrm{C}(10)-\mathrm{C}(11)-\mathrm{H}(11)$ & 109.1 \\
\hline $\mathrm{C}(17)-\mathrm{C}(11)-\mathrm{H}(11)$ & 109.1 \\
\hline $\mathrm{C}(11)-\mathrm{C}(12)-\mathrm{C}(13)$ & $116.51(16)$ \\
\hline$C(11)-C(12)-H(12 A)$ & 108.2 \\
\hline $\mathrm{C}(13)-\mathrm{C}(12)-\mathrm{H}(12 \mathrm{~A})$ & 108.2 \\
\hline $\mathrm{C}(11)-\mathrm{C}(12)-\mathrm{H}(12 \mathrm{~B})$ & 108.2 \\
\hline $\mathrm{C}(13)-\mathrm{C}(12)-\mathrm{H}(12 \mathrm{~B})$ & 108.2 \\
\hline
\end{tabular}

\begin{tabular}{|c|c|}
\hline $\mathrm{H}(12 \mathrm{~A})-\mathrm{C}(12)-\mathrm{H}(12 \mathrm{~B})$ & 107.3 \\
\hline$C(14)-C(13)-C(12)$ & $116.40(17)$ \\
\hline $\mathrm{C}(14)-\mathrm{C}(13)-\mathrm{H}(13 \mathrm{~A})$ & 108.2 \\
\hline $\mathrm{C}(12)-\mathrm{C}(13)-\mathrm{H}(13 \mathrm{~A})$ & 108.2 \\
\hline $\mathrm{C}(14)-\mathrm{C}(13)-\mathrm{H}(13 \mathrm{~B})$ & 108.2 \\
\hline$C(12)-C(13)-H(13 B)$ & 108.2 \\
\hline $\mathrm{H}(13 \mathrm{~A})-\mathrm{C}(13)-\mathrm{H}(13 \mathrm{~B})$ & 107.3 \\
\hline$C(15 A)-C(14)-C(13)$ & $121.6(8)$ \\
\hline$C(13)-C(14)-C(15)$ & $116.50(18)$ \\
\hline $\mathrm{C}(13)-\mathrm{C}(14)-\mathrm{H}(14 \mathrm{~A})$ & 108.2 \\
\hline $\mathrm{C}(15)-\mathrm{C}(14)-\mathrm{H}(14 \mathrm{~A})$ & 108.2 \\
\hline $\mathrm{C}(13)-\mathrm{C}(14)-\mathrm{H}(14 \mathrm{~B})$ & 108.2 \\
\hline $\mathrm{C}(15)-\mathrm{C}(14)-\mathrm{H}(14 \mathrm{~B})$ & 108.2 \\
\hline $\mathrm{H}(14 \mathrm{~A})-\mathrm{C}(14)-\mathrm{H}(14 \mathrm{~B})$ & 107.3 \\
\hline $\mathrm{C}(15 \mathrm{~A})-\mathrm{C}(14)-\mathrm{H}(14 \mathrm{C})$ & 106.9 \\
\hline $\mathrm{C}(13)-\mathrm{C}(14)-\mathrm{H}(14 \mathrm{C})$ & 106.9 \\
\hline $\mathrm{C}(15 \mathrm{~A})-\mathrm{C}(14)-\mathrm{H}(14 \mathrm{D})$ & 106.9 \\
\hline $\mathrm{C}(13)-\mathrm{C}(14)-\mathrm{H}(14 \mathrm{D})$ & 106.9 \\
\hline $\mathrm{H}(14 \mathrm{C})-\mathrm{C}(14)-\mathrm{H}(14 \mathrm{D})$ & 106.7 \\
\hline$C(14)-C(15)-C(16)$ & $111.75(17)$ \\
\hline $\mathrm{C}(14)-\mathrm{C}(15)-\mathrm{H}(15 \mathrm{~A})$ & 109.3 \\
\hline $\mathrm{C}(16)-\mathrm{C}(15)-\mathrm{H}(15 \mathrm{~A})$ & 109.3 \\
\hline $\mathrm{C}(14)-\mathrm{C}(15)-\mathrm{H}(15 \mathrm{~B})$ & 109.3 \\
\hline $\mathrm{C}(16)-\mathrm{C}(15)-\mathrm{H}(15 \mathrm{~B})$ & 109.3 \\
\hline $\mathrm{H}(15 \mathrm{~A})-\mathrm{C}(15)-\mathrm{H}(15 \mathrm{~B})$ & 107.9 \\
\hline$C(14)-C(15 A)-C(16)$ & $111.4(10)$ \\
\hline $\mathrm{C}(14)-\mathrm{C}(15 \mathrm{~A})-\mathrm{H}(15 \mathrm{C})$ & 109.3 \\
\hline$C(16)-C(15 A)-H(15 C)$ & 109.3 \\
\hline $\mathrm{C}(14)-\mathrm{C}(15 \mathrm{~A})-\mathrm{H}(15 \mathrm{D})$ & 109.3 \\
\hline$C(16)-C(15 A)-H(15 D)$ & 109.3 \\
\hline $\mathrm{H}(15 \mathrm{C})-\mathrm{C}(15 \mathrm{~A})-\mathrm{H}(15 \mathrm{D})$ & 108.0 \\
\hline$C(17)-C(16)-C(15)$ & $114.60(17)$ \\
\hline$C(17)-C(16)-C(15 A)$ & $105.3(8)$ \\
\hline $\mathrm{C}(17)-\mathrm{C}(16)-\mathrm{H}(16 \mathrm{~A})$ & 108.6 \\
\hline $\mathrm{C}(15)-\mathrm{C}(16)-\mathrm{H}(16 \mathrm{~A})$ & 108.6 \\
\hline $\mathrm{C}(17)-\mathrm{C}(16)-\mathrm{H}(16 \mathrm{~B})$ & 108.6 \\
\hline $\mathrm{C}(15)-\mathrm{C}(16)-\mathrm{H}(16 \mathrm{~B})$ & 108.6 \\
\hline $\mathrm{H}(16 \mathrm{~A})-\mathrm{C}(16)-\mathrm{H}(16 \mathrm{~B})$ & 107.6 \\
\hline $\mathrm{C}(17)-\mathrm{C}(16)-\mathrm{H}(16 \mathrm{C})$ & 110.7 \\
\hline$C(15 A)-C(16)-H(16 C)$ & 110.7 \\
\hline
\end{tabular}




\begin{tabular}{|c|c|c|c|}
\hline$C(17)-C(16)-H(16 D)$ & 110.7 & $\mathrm{C}(37)-\mathrm{C}(28)-\mathrm{C}(29)$ & $104.5(5)$ \\
\hline$C(15 A)-C(16)-H(16 D)$ & 110.7 & $\mathrm{O}(24)-\mathrm{C}(28)-\mathrm{H}(28)$ & 111.4 \\
\hline$H(16 C)-C(16)-H(16 D)$ & 108.8 & $\mathrm{C}(37)-\mathrm{C}(28)-\mathrm{H}(28)$ & 111.4 \\
\hline$C(8)-C(17)-C(16)$ & $115.07(15)$ & $\mathrm{C}(29)-\mathrm{C}(28)-\mathrm{H}(28)$ & 111.4 \\
\hline$C(8)-C(17)-C(11)$ & $103.46(15)$ & $\mathrm{C}(30)-\mathrm{C}(29)-\mathrm{C}(28)$ & $105.8(5)$ \\
\hline$C(16)-C(17)-C(11)$ & $118.87(16)$ & $\mathrm{C}(30)-\mathrm{C}(29)-\mathrm{H}(29 \mathrm{~A})$ & 110.6 \\
\hline C(8)-C(17)-H(17) & 106.2 & $\mathrm{C}(28)-\mathrm{C}(29)-\mathrm{H}(29 \mathrm{~A})$ & 110.6 \\
\hline $\mathrm{C}(16)-\mathrm{C}(17)-\mathrm{H}(17)$ & 106.2 & $\mathrm{C}(30)-\mathrm{C}(29)-\mathrm{H}(29 \mathrm{~B})$ & 110.6 \\
\hline $\mathrm{C}(11)-\mathrm{C}(17)-\mathrm{H}(17)$ & 106.2 & $\mathrm{C}(28)-\mathrm{C}(29)-\mathrm{H}(29 \mathrm{~B})$ & 110.6 \\
\hline $\mathrm{O}(21)-\mathrm{N}(21)-\mathrm{O}(22)$ & $123.73(15)$ & $\mathrm{H}(29 \mathrm{~A})-\mathrm{C}(29)-\mathrm{H}(29 \mathrm{~B})$ & 108.7 \\
\hline $\mathrm{O}(21)-\mathrm{N}(21)-\mathrm{C}(21)$ & $118.15(15)$ & $C(31)-C(30)-C(29)$ & $105.9(5)$ \\
\hline $\mathrm{O}(22)-\mathrm{N}(21)-\mathrm{C}(21)$ & $118.12(15)$ & $\mathrm{C}(31)-\mathrm{C}(30)-\mathrm{H}(30 \mathrm{~A})$ & 110.6 \\
\hline$C(26)-C(21)-C(22)$ & $122.87(16)$ & $\mathrm{C}(29)-\mathrm{C}(30)-\mathrm{H}(30 \mathrm{~A})$ & 110.6 \\
\hline $\mathrm{C}(26)-\mathrm{C}(21)-\mathrm{N}(21)$ & $118.64(15)$ & $\mathrm{C}(31)-\mathrm{C}(30)-\mathrm{H}(30 \mathrm{~B})$ & 110.6 \\
\hline $\mathrm{C}(22)-\mathrm{C}(21)-\mathrm{N}(21)$ & $118.49(16)$ & $\mathrm{C}(29)-\mathrm{C}(30)-\mathrm{H}(30 \mathrm{~B})$ & 110.6 \\
\hline$C(23)-C(22)-C(21)$ & $117.96(17)$ & $\mathrm{H}(30 \mathrm{~A})-\mathrm{C}(30)-\mathrm{H}(30 \mathrm{~B})$ & 108.7 \\
\hline $\mathrm{C}(23)-\mathrm{C}(22)-\mathrm{H}(22)$ & 121.0 & $\mathrm{C}(37)-\mathrm{C}(31)-\mathrm{C}(30)$ & $101.0(5)$ \\
\hline $\mathrm{C}(21)-\mathrm{C}(22)-\mathrm{H}(22)$ & 121.0 & $C(37)-C(31)-C(32)$ & $113.7(7)$ \\
\hline $\mathrm{C}(22)-\mathrm{C}(23)-\mathrm{C}(24)$ & $120.71(17)$ & $C(30)-C(31)-C(32)$ & $113.4(7)$ \\
\hline $\mathrm{C}(22)-\mathrm{C}(23)-\mathrm{H}(23)$ & 119.6 & $\mathrm{C}(37)-\mathrm{C}(31)-\mathrm{H}(31)$ & 109.5 \\
\hline $\mathrm{C}(24)-\mathrm{C}(23)-\mathrm{H}(23)$ & 119.6 & $\mathrm{C}(30)-\mathrm{C}(31)-\mathrm{H}(31)$ & 109.5 \\
\hline$C(23)-C(24)-C(25)$ & $119.98(16)$ & $\mathrm{C}(32)-\mathrm{C}(31)-\mathrm{H}(31)$ & 109.5 \\
\hline$C(23)-C(24)-C(27)$ & $117.72(16)$ & $\mathrm{C}(33)-\mathrm{C}(32)-\mathrm{C}(31)$ & $113.8(7)$ \\
\hline$C(25)-C(24)-C(27)$ & $122.30(17)$ & $\mathrm{C}(33)-\mathrm{C}(32)-\mathrm{H}(32 \mathrm{~A})$ & 108.8 \\
\hline$C(26)-C(25)-C(24)$ & $120.12(17)$ & $\mathrm{C}(31)-\mathrm{C}(32)-\mathrm{H}(32 \mathrm{~A})$ & 108.8 \\
\hline $\mathrm{C}(26)-\mathrm{C}(25)-\mathrm{H}(25)$ & 119.9 & $\mathrm{C}(33)-\mathrm{C}(32)-\mathrm{H}(32 \mathrm{~B})$ & 108.8 \\
\hline $\mathrm{C}(24)-\mathrm{C}(25)-\mathrm{H}(25)$ & 119.9 & $\mathrm{C}(31)-\mathrm{C}(32)-\mathrm{H}(32 \mathrm{~B})$ & 108.8 \\
\hline$C(21)-C(26)-C(25)$ & $118.33(16)$ & $\mathrm{H}(32 \mathrm{~A})-\mathrm{C}(32)-\mathrm{H}(32 \mathrm{~B})$ & 107.7 \\
\hline $\mathrm{C}(21)-\mathrm{C}(26)-\mathrm{H}(26)$ & 120.8 & $C(34)-C(33)-C(32)$ & $120.5(8)$ \\
\hline $\mathrm{C}(25)-\mathrm{C}(26)-\mathrm{H}(26)$ & 120.8 & $\mathrm{C}(34)-\mathrm{C}(33)-\mathrm{H}(33 \mathrm{~A})$ & 107.2 \\
\hline $\mathrm{O}(24 \mathrm{~B})-\mathrm{C}(27)-\mathrm{O}(23)$ & $120(2)$ & $\mathrm{C}(32)-\mathrm{C}(33)-\mathrm{H}(33 \mathrm{~A})$ & 107.2 \\
\hline $\mathrm{O}(23)-\mathrm{C}(27)-\mathrm{O}(24 \mathrm{~A})$ & $125.4(17)$ & $\mathrm{C}(34)-\mathrm{C}(33)-\mathrm{H}(33 \mathrm{~B})$ & 107.2 \\
\hline $\mathrm{O}(23)-\mathrm{C}(27)-\mathrm{O}(24)$ & $124.3(7)$ & $\mathrm{C}(32)-\mathrm{C}(33)-\mathrm{H}(33 \mathrm{~B})$ & 107.2 \\
\hline $\mathrm{O}(24 \mathrm{~B})-\mathrm{C}(27)-\mathrm{C}(24)$ & $116(2)$ & $\mathrm{H}(33 \mathrm{~A})-\mathrm{C}(33)-\mathrm{H}(33 \mathrm{~B})$ & 106.8 \\
\hline $\mathrm{O}(23)-\mathrm{C}(27)-\mathrm{C}(24)$ & $123.84(18)$ & $C(33)-C(34)-C(35)$ & $119.3(9)$ \\
\hline $\mathrm{O}(24 \mathrm{~A})-\mathrm{C}(27)-\mathrm{C}(24)$ & $110.6(17)$ & $\mathrm{C}(33)-\mathrm{C}(34)-\mathrm{H}(34 \mathrm{~A})$ & 107.5 \\
\hline $\mathrm{O}(24)-\mathrm{C}(27)-\mathrm{C}(24)$ & $111.8(8)$ & $\mathrm{C}(35)-\mathrm{C}(34)-\mathrm{H}(34 \mathrm{~A})$ & 107.5 \\
\hline $\mathrm{C}(27)-\mathrm{O}(24)-\mathrm{C}(28)$ & $118.2(15)$ & $\mathrm{C}(33)-\mathrm{C}(34)-\mathrm{H}(34 \mathrm{~B})$ & 107.5 \\
\hline $\mathrm{O}(24)-\mathrm{C}(28)-\mathrm{C}(37)$ & $107.7(8)$ & $\mathrm{C}(35)-\mathrm{C}(34)-\mathrm{H}(34 \mathrm{~B})$ & 107.5 \\
\hline $\mathrm{O}(24)-\mathrm{C}(28)-\mathrm{C}(29)$ & $110.3(8)$ & $\mathrm{H}(34 \mathrm{~A})-\mathrm{C}(34)-\mathrm{H}(34 \mathrm{~B})$ & 107.0 \\
\hline
\end{tabular}




\begin{tabular}{|c|c|c|c|}
\hline $\mathrm{C}(34)-\mathrm{C}(35)-\mathrm{C}(36)$ & $110.6(7)$ & $\mathrm{C}(30 \mathrm{~A})-\mathrm{C}(31 \mathrm{~A})-\mathrm{H}(31 \mathrm{~A})$ & 107.1 \\
\hline $\mathrm{C}(34)-\mathrm{C}(35)-\mathrm{H}(35 \mathrm{~A})$ & 109.5 & $\mathrm{C}(37 \mathrm{~A})-\mathrm{C}(31 \mathrm{~A})-\mathrm{H}(31 \mathrm{~A})$ & 107.1 \\
\hline $\mathrm{C}(36)-\mathrm{C}(35)-\mathrm{H}(35 \mathrm{~A})$ & 109.5 & $\mathrm{C}(32 \mathrm{~A})-\mathrm{C}(31 \mathrm{~A})-\mathrm{H}(31 \mathrm{~A})$ & 107.1 \\
\hline $\mathrm{C}(34)-\mathrm{C}(35)-\mathrm{H}(35 \mathrm{~B})$ & 109.5 & $C(33 A)-C(32 A)-C(31 A)$ & $115.8(16)$ \\
\hline $\mathrm{C}(36)-\mathrm{C}(35)-\mathrm{H}(35 \mathrm{~B})$ & 109.5 & $\mathrm{C}(33 \mathrm{~A})-\mathrm{C}(32 \mathrm{~A})-\mathrm{H}(32 \mathrm{C})$ & 108.3 \\
\hline $\mathrm{H}(35 \mathrm{~A})-\mathrm{C}(35)-\mathrm{H}(35 \mathrm{~B})$ & 108.1 & $\mathrm{C}(31 \mathrm{~A})-\mathrm{C}(32 \mathrm{~A})-\mathrm{H}(32 \mathrm{C})$ & 108.3 \\
\hline $\mathrm{C}(35)-\mathrm{C}(36)-\mathrm{C}(37)$ & $113.2(8)$ & $\mathrm{C}(33 \mathrm{~A})-\mathrm{C}(32 \mathrm{~A})-\mathrm{H}(32 \mathrm{D})$ & 108.3 \\
\hline$C(35)-C(36)-H(36 A)$ & 108.9 & $\mathrm{C}(31 \mathrm{~A})-\mathrm{C}(32 \mathrm{~A})-\mathrm{H}(32 \mathrm{D})$ & 108.3 \\
\hline $\mathrm{C}(37)-\mathrm{C}(36)-\mathrm{H}(36 \mathrm{~A})$ & 108.9 & $\mathrm{H}(32 \mathrm{C})-\mathrm{C}(32 \mathrm{~A})-\mathrm{H}(32 \mathrm{D})$ & 107.4 \\
\hline $\mathrm{C}(35)-\mathrm{C}(36)-\mathrm{H}(36 \mathrm{~B})$ & 108.9 & $C(34 \mathrm{~A})-\mathrm{C}(33 \mathrm{~A})-\mathrm{C}(32 \mathrm{~A})$ & $116.3(17)$ \\
\hline $\mathrm{C}(37)-\mathrm{C}(36)-\mathrm{H}(36 \mathrm{~B})$ & 108.9 & $\mathrm{C}(34 \mathrm{~A})-\mathrm{C}(33 \mathrm{~A})-\mathrm{H}(33 \mathrm{C})$ & 108.2 \\
\hline $\mathrm{H}(36 \mathrm{~A})-\mathrm{C}(36)-\mathrm{H}(36 \mathrm{~B})$ & 107.8 & $\mathrm{C}(32 \mathrm{~A})-\mathrm{C}(33 \mathrm{~A})-\mathrm{H}(33 \mathrm{C})$ & 108.2 \\
\hline $\mathrm{C}(28)-\mathrm{C}(37)-\mathrm{C}(31)$ & $105.1(5)$ & $\mathrm{C}(34 \mathrm{~A})-\mathrm{C}(33 \mathrm{~A})-\mathrm{H}(33 \mathrm{D})$ & 108.2 \\
\hline $\mathrm{C}(28)-\mathrm{C}(37)-\mathrm{C}(36)$ & $113.4(7)$ & $\mathrm{C}(32 \mathrm{~A})-\mathrm{C}(33 \mathrm{~A})-\mathrm{H}(33 \mathrm{D})$ & 108.2 \\
\hline$C(31)-C(37)-C(36)$ & $118.6(7)$ & $\mathrm{H}(33 \mathrm{C})-\mathrm{C}(33 \mathrm{~A})-\mathrm{H}(33 \mathrm{D})$ & 107.4 \\
\hline C(28)-C(37)-H(37) & 106.3 & $C(33 A)-C(34 A)-C(35 A)$ & $113.2(18)$ \\
\hline $\mathrm{C}(31)-\mathrm{C}(37)-\mathrm{H}(37)$ & 106.3 & $\mathrm{C}(33 \mathrm{~A})-\mathrm{C}(34 \mathrm{~A})-\mathrm{H}(34 \mathrm{C})$ & 108.9 \\
\hline C(36)-C(37)-H(37) & 106.3 & $\mathrm{C}(35 \mathrm{~A})-\mathrm{C}(34 \mathrm{~A})-\mathrm{H}(34 \mathrm{C})$ & 108.9 \\
\hline $\mathrm{C}(27)-\mathrm{O}(24 \mathrm{~A})-\mathrm{C}(28 \mathrm{~A})$ & 111(3) & $\mathrm{C}(33 \mathrm{~A})-\mathrm{C}(34 \mathrm{~A})-\mathrm{H}(34 \mathrm{D})$ & 108.9 \\
\hline $\mathrm{O}(24 \mathrm{~A})-\mathrm{C}(28 \mathrm{~A})-\mathrm{C}(37 \mathrm{~A})$ & $105.7(18)$ & $\mathrm{C}(35 \mathrm{~A})-\mathrm{C}(34 \mathrm{~A})-\mathrm{H}(34 \mathrm{D})$ & 108.9 \\
\hline $\mathrm{O}(24 \mathrm{~A})-\mathrm{C}(28 \mathrm{~A})-\mathrm{C}(29 \mathrm{~A})$ & $109(2)$ & $\mathrm{H}(34 \mathrm{C})-\mathrm{C}(34 \mathrm{~A})-\mathrm{H}(34 \mathrm{D})$ & 107.8 \\
\hline$C(37 A)-C(28 A)-C(29 A)$ & $104.1(11)$ & $C(36 A)-C(35 A)-C(34 A)$ & $111.3(17)$ \\
\hline $\mathrm{O}(24 \mathrm{~A})-\mathrm{C}(28 \mathrm{~A})-\mathrm{H}(28 \mathrm{~A})$ & 112.5 & $\mathrm{C}(36 \mathrm{~A})-\mathrm{C}(35 \mathrm{~A})-\mathrm{H}(35 \mathrm{C})$ & 109.4 \\
\hline $\mathrm{C}(37 \mathrm{~A})-\mathrm{C}(28 \mathrm{~A})-\mathrm{H}(28 \mathrm{~A})$ & 112.5 & $\mathrm{C}(34 \mathrm{~A})-\mathrm{C}(35 \mathrm{~A})-\mathrm{H}(35 \mathrm{C})$ & 109.4 \\
\hline $\mathrm{C}(29 \mathrm{~A})-\mathrm{C}(28 \mathrm{~A})-\mathrm{H}(28 \mathrm{~A})$ & 112.5 & $\mathrm{C}(36 \mathrm{~A})-\mathrm{C}(35 \mathrm{~A})-\mathrm{H}(35 \mathrm{D})$ & 109.4 \\
\hline$C(30 A)-C(29 A)-C(28 A)$ & $106.6(11)$ & $\mathrm{C}(34 \mathrm{~A})-\mathrm{C}(35 \mathrm{~A})-\mathrm{H}(35 \mathrm{D})$ & 109.4 \\
\hline$C(30 A)-C(29 A)-H(29 C)$ & 110.4 & $\mathrm{H}(35 \mathrm{C})-\mathrm{C}(35 \mathrm{~A})-\mathrm{H}(35 \mathrm{D})$ & 108.0 \\
\hline $\mathrm{C}(28 \mathrm{~A})-\mathrm{C}(29 \mathrm{~A})-\mathrm{H}(29 \mathrm{C})$ & 110.4 & $C(37 A)-C(36 A)-C(35 A)$ & $116.9(16)$ \\
\hline$C(30 A)-C(29 A)-H(29 D)$ & 110.4 & $\mathrm{C}(37 \mathrm{~A})-\mathrm{C}(36 \mathrm{~A})-\mathrm{H}(36 \mathrm{C})$ & 108.1 \\
\hline $\mathrm{C}(28 \mathrm{~A})-\mathrm{C}(29 \mathrm{~A})-\mathrm{H}(29 \mathrm{D})$ & 110.4 & $\mathrm{C}(35 \mathrm{~A})-\mathrm{C}(36 \mathrm{~A})-\mathrm{H}(36 \mathrm{C})$ & 108.1 \\
\hline $\mathrm{H}(29 \mathrm{C})-\mathrm{C}(29 \mathrm{~A})-\mathrm{H}(29 \mathrm{D})$ & 108.6 & $\mathrm{C}(37 \mathrm{~A})-\mathrm{C}(36 \mathrm{~A})-\mathrm{H}(36 \mathrm{D})$ & 108.1 \\
\hline$C(29 A)-C(30 A)-C(31 A)$ & $105.8(11)$ & $\mathrm{C}(35 \mathrm{~A})-\mathrm{C}(36 \mathrm{~A})-\mathrm{H}(36 \mathrm{D})$ & 108.1 \\
\hline $\mathrm{C}(29 \mathrm{~A})-\mathrm{C}(30 \mathrm{~A})-\mathrm{H}(30 \mathrm{C})$ & 110.6 & $\mathrm{H}(36 \mathrm{C})-\mathrm{C}(36 \mathrm{~A})-\mathrm{H}(36 \mathrm{D})$ & 107.3 \\
\hline $\mathrm{C}(31 \mathrm{~A})-\mathrm{C}(30 \mathrm{~A})-\mathrm{H}(30 \mathrm{C})$ & 110.6 & $C(36 \mathrm{~A})-\mathrm{C}(37 \mathrm{~A})-\mathrm{C}(28 \mathrm{~A})$ & $115.3(16)$ \\
\hline $\mathrm{C}(29 \mathrm{~A})-\mathrm{C}(30 \mathrm{~A})-\mathrm{H}(30 \mathrm{D})$ & 110.6 & $C(36 A)-C(37 A)-C(31 A)$ & $118.5(15)$ \\
\hline $\mathrm{C}(31 \mathrm{~A})-\mathrm{C}(30 \mathrm{~A})-\mathrm{H}(30 \mathrm{D})$ & 110.6 & $\mathrm{C}(28 \mathrm{~A})-\mathrm{C}(37 \mathrm{~A})-\mathrm{C}(31 \mathrm{~A})$ & $103.6(12)$ \\
\hline $\mathrm{H}(30 \mathrm{C})-\mathrm{C}(30 \mathrm{~A})-\mathrm{H}(30 \mathrm{D})$ & 108.7 & $\mathrm{C}(36 \mathrm{~A})-\mathrm{C}(37 \mathrm{~A})-\mathrm{H}(37 \mathrm{~A})$ & 106.2 \\
\hline$C(30 A)-C(31 A)-C(37 A)$ & $101.6(12)$ & $\mathrm{C}(28 \mathrm{~A})-\mathrm{C}(37 \mathrm{~A})-\mathrm{H}(37 \mathrm{~A})$ & 106.2 \\
\hline$C(30 A)-C(31 A)-C(32 A)$ & $120.2(16)$ & $\mathrm{C}(31 \mathrm{~A})-\mathrm{C}(37 \mathrm{~A})-\mathrm{H}(37 \mathrm{~A})$ & 106.2 \\
\hline$C(37 \mathrm{~A})-\mathrm{C}(31 \mathrm{~A})-\mathrm{C}(32 \mathrm{~A})$ & $113.0(17)$ & $\mathrm{C}(27)-\mathrm{O}(24 \mathrm{~B})-\mathrm{C}(28 \mathrm{~B})$ & $118(4)$ \\
\hline
\end{tabular}




\begin{tabular}{|c|c|c|c|}
\hline $\mathrm{O}(24 \mathrm{~B})-\mathrm{C}(28 \mathrm{~B})-\mathrm{C}(37 \mathrm{~B})$ & $102.7(19)$ & $\mathrm{C}(35 \mathrm{~B})-\mathrm{C}(34 \mathrm{~B})-\mathrm{H}(34 \mathrm{~F})$ & 108.0 \\
\hline $\mathrm{O}(24 \mathrm{~B})-\mathrm{C}(28 \mathrm{~B})-\mathrm{C}(29 \mathrm{~B})$ & $108(3)$ & $\mathrm{H}(34 \mathrm{E})-\mathrm{C}(34 \mathrm{~B})-\mathrm{H}(34 \mathrm{~F})$ & 107.2 \\
\hline $\mathrm{C}(37 \mathrm{~B})-\mathrm{C}(28 \mathrm{~B})-\mathrm{C}(29 \mathrm{~B})$ & $103.5(12)$ & $\mathrm{C}(34 \mathrm{~B})-\mathrm{C}(35 \mathrm{~B})-\mathrm{C}(36 \mathrm{~B})$ & $115.3(12)$ \\
\hline $\mathrm{O}(24 \mathrm{~B})-\mathrm{C}(28 \mathrm{~B})-\mathrm{H}(28 \mathrm{~B})$ & 113.7 & $\mathrm{C}(34 \mathrm{~B})-\mathrm{C}(35 \mathrm{~B})-\mathrm{H}(35 \mathrm{E})$ & 108.5 \\
\hline $\mathrm{C}(37 \mathrm{~B})-\mathrm{C}(28 \mathrm{~B})-\mathrm{H}(28 \mathrm{~B})$ & 113.7 & $\mathrm{C}(36 \mathrm{~B})-\mathrm{C}(35 \mathrm{~B})-\mathrm{H}(35 \mathrm{E})$ & 108.5 \\
\hline $\mathrm{C}(29 \mathrm{~B})-\mathrm{C}(28 \mathrm{~B})-\mathrm{H}(28 \mathrm{~B})$ & 113.7 & $\mathrm{C}(34 \mathrm{~B})-\mathrm{C}(35 \mathrm{~B})-\mathrm{H}(35 \mathrm{~F})$ & 108.5 \\
\hline$C(30 B)-C(29 B)-C(28 B)$ & $106.5(11)$ & $\mathrm{C}(36 \mathrm{~B})-\mathrm{C}(35 \mathrm{~B})-\mathrm{H}(35 \mathrm{~F})$ & 108.5 \\
\hline$C(30 B)-C(29 B)-H(29 E)$ & 110.4 & $\mathrm{H}(35 \mathrm{E})-\mathrm{C}(35 \mathrm{~B})-\mathrm{H}(35 \mathrm{~F})$ & 107.5 \\
\hline $\mathrm{C}(28 \mathrm{~B})-\mathrm{C}(29 \mathrm{~B})-\mathrm{H}(29 \mathrm{E})$ & 110.4 & $\mathrm{C}(37 \mathrm{~B})-\mathrm{C}(36 \mathrm{~B})-\mathrm{C}(35 \mathrm{~B})$ & $115.7(14)$ \\
\hline $\mathrm{C}(30 \mathrm{~B})-\mathrm{C}(29 \mathrm{~B})-\mathrm{H}(29 \mathrm{~F})$ & 110.4 & $\mathrm{C}(37 \mathrm{~B})-\mathrm{C}(36 \mathrm{~B})-\mathrm{H}(36 \mathrm{E})$ & 108.4 \\
\hline $\mathrm{C}(28 \mathrm{~B})-\mathrm{C}(29 \mathrm{~B})-\mathrm{H}(29 \mathrm{~F})$ & 110.4 & $\mathrm{C}(35 \mathrm{~B})-\mathrm{C}(36 \mathrm{~B})-\mathrm{H}(36 \mathrm{E})$ & 108.4 \\
\hline $\mathrm{H}(29 \mathrm{E})-\mathrm{C}(29 \mathrm{~B})-\mathrm{H}(29 \mathrm{~F})$ & 108.6 & $\mathrm{C}(37 \mathrm{~B})-\mathrm{C}(36 \mathrm{~B})-\mathrm{H}(36 \mathrm{~F})$ & 108.4 \\
\hline$C(29 B)-C(30 B)-C(31 B)$ & $105.1(10)$ & $\mathrm{C}(35 \mathrm{~B})-\mathrm{C}(36 \mathrm{~B})-\mathrm{H}(36 \mathrm{~F})$ & 108.4 \\
\hline$C(29 B)-C(30 B)-H(30 E)$ & 110.7 & $\mathrm{H}(36 \mathrm{E})-\mathrm{C}(36 \mathrm{~B})-\mathrm{H}(36 \mathrm{~F})$ & 107.4 \\
\hline $\mathrm{C}(31 \mathrm{~B})-\mathrm{C}(30 \mathrm{~B})-\mathrm{H}(30 \mathrm{E})$ & 110.7 & $\mathrm{C}(36 \mathrm{~B})-\mathrm{C}(37 \mathrm{~B})-\mathrm{C}(31 \mathrm{~B})$ & $117.9(14)$ \\
\hline $\mathrm{C}(29 \mathrm{~B})-\mathrm{C}(30 \mathrm{~B})-\mathrm{H}(30 \mathrm{~F})$ & 110.7 & $\mathrm{C}(36 \mathrm{~B})-\mathrm{C}(37 \mathrm{~B})-\mathrm{C}(28 \mathrm{~B})$ & $114.5(13)$ \\
\hline $\mathrm{C}(31 \mathrm{~B})-\mathrm{C}(30 \mathrm{~B})-\mathrm{H}(30 \mathrm{~F})$ & 110.7 & $\mathrm{C}(31 \mathrm{~B})-\mathrm{C}(37 \mathrm{~B})-\mathrm{C}(28 \mathrm{~B})$ & $103.3(12)$ \\
\hline $\mathrm{H}(30 \mathrm{E})-\mathrm{C}(30 \mathrm{~B})-\mathrm{H}(30 \mathrm{~F})$ & 108.8 & $\mathrm{C}(36 \mathrm{~B})-\mathrm{C}(37 \mathrm{~B})-\mathrm{H}(37 \mathrm{~B})$ & 106.8 \\
\hline $\mathrm{C}(37 \mathrm{~B})-\mathrm{C}(31 \mathrm{~B})-\mathrm{C}(30 \mathrm{~B})$ & $100.3(12)$ & $\mathrm{C}(31 \mathrm{~B})-\mathrm{C}(37 \mathrm{~B})-\mathrm{H}(37 \mathrm{~B})$ & 106.8 \\
\hline$C(37 B)-C(31 B)-C(32 B)$ & $114.2(14)$ & $\mathrm{C}(28 \mathrm{~B})-\mathrm{C}(37 \mathrm{~B})-\mathrm{H}(37 \mathrm{~B})$ & 106.8 \\
\hline $\mathrm{C}(30 \mathrm{~B})-\mathrm{C}(31 \mathrm{~B})-\mathrm{C}(32 \mathrm{~B})$ & $111.8(13)$ & $\mathrm{C}(47)-\mathrm{O}(44)-\mathrm{C}(48)$ & $116.76(14)$ \\
\hline $\mathrm{C}(37 \mathrm{~B})-\mathrm{C}(31 \mathrm{~B})-\mathrm{H}(31 \mathrm{~B})$ & 110.1 & $\mathrm{O}(42)-\mathrm{N}(41)-\mathrm{O}(41)$ & $123.51(16)$ \\
\hline $\mathrm{C}(30 \mathrm{~B})-\mathrm{C}(31 \mathrm{~B})-\mathrm{H}(31 \mathrm{~B})$ & 110.1 & $\mathrm{O}(42)-\mathrm{N}(41)-\mathrm{C}(41)$ & $118.03(15)$ \\
\hline $\mathrm{C}(32 \mathrm{~B})-\mathrm{C}(31 \mathrm{~B})-\mathrm{H}(31 \mathrm{~B})$ & 110.1 & $\mathrm{O}(41)-\mathrm{N}(41)-\mathrm{C}(41)$ & $118.44(14)$ \\
\hline $\mathrm{C}(33 \mathrm{~B})-\mathrm{C}(32 \mathrm{~B})-\mathrm{C}(31 \mathrm{~B})$ & $113.1(13)$ & $\mathrm{C}(46)-\mathrm{C}(41)-\mathrm{C}(42)$ & $122.61(16)$ \\
\hline $\mathrm{C}(33 \mathrm{~B})-\mathrm{C}(32 \mathrm{~B})-\mathrm{H}(32 \mathrm{E})$ & 109.0 & $\mathrm{C}(46)-\mathrm{C}(41)-\mathrm{N}(41)$ & $118.67(15)$ \\
\hline $\mathrm{C}(31 \mathrm{~B})-\mathrm{C}(32 \mathrm{~B})-\mathrm{H}(32 \mathrm{E})$ & 109.0 & $\mathrm{C}(42)-\mathrm{C}(41)-\mathrm{N}(41)$ & $118.72(15)$ \\
\hline $\mathrm{C}(33 \mathrm{~B})-\mathrm{C}(32 \mathrm{~B})-\mathrm{H}(32 \mathrm{~F})$ & 109.0 & $\mathrm{C}(43)-\mathrm{C}(42)-\mathrm{C}(41)$ & $118.50(16)$ \\
\hline $\mathrm{C}(31 \mathrm{~B})-\mathrm{C}(32 \mathrm{~B})-\mathrm{H}(32 \mathrm{~F})$ & 109.0 & $\mathrm{C}(43)-\mathrm{C}(42)-\mathrm{H}(42)$ & 120.8 \\
\hline $\mathrm{H}(32 \mathrm{E})-\mathrm{C}(32 \mathrm{~B})-\mathrm{H}(32 \mathrm{~F})$ & 107.8 & $\mathrm{C}(41)-\mathrm{C}(42)-\mathrm{H}(42)$ & 120.8 \\
\hline $\mathrm{C}(34 \mathrm{~B})-\mathrm{C}(33 \mathrm{~B})-\mathrm{C}(32 \mathrm{~B})$ & $117.1(16)$ & $C(42)-C(43)-C(44)$ & $120.37(16)$ \\
\hline $\mathrm{C}(34 \mathrm{~B})-\mathrm{C}(33 \mathrm{~B})-\mathrm{H}(33 \mathrm{E})$ & 108.0 & $\mathrm{C}(42)-\mathrm{C}(43)-\mathrm{H}(43)$ & 119.8 \\
\hline$C(32 B)-C(33 B)-H(33 E)$ & 108.0 & $\mathrm{C}(44)-\mathrm{C}(43)-\mathrm{H}(43)$ & 119.8 \\
\hline $\mathrm{C}(34 \mathrm{~B})-\mathrm{C}(33 \mathrm{~B})-\mathrm{H}(33 \mathrm{~F})$ & 108.0 & $\mathrm{C}(43)-\mathrm{C}(44)-\mathrm{C}(45)$ & $119.75(16)$ \\
\hline $\mathrm{C}(32 \mathrm{~B})-\mathrm{C}(33 \mathrm{~B})-\mathrm{H}(33 \mathrm{~F})$ & 108.0 & $C(43)-C(44)-C(47)$ & $122.24(15)$ \\
\hline $\mathrm{H}(33 \mathrm{E})-\mathrm{C}(33 \mathrm{~B})-\mathrm{H}(33 \mathrm{~F})$ & 107.3 & $\mathrm{C}(45)-\mathrm{C}(44)-\mathrm{C}(47)$ & $118.01(15)$ \\
\hline$C(33 B)-C(34 B)-C(35 B)$ & $117.2(14)$ & $C(46)-C(45)-C(44)$ & $120.69(16)$ \\
\hline $\mathrm{C}(33 \mathrm{~B})-\mathrm{C}(34 \mathrm{~B})-\mathrm{H}(34 \mathrm{E})$ & 108.0 & $\mathrm{C}(46)-\mathrm{C}(45)-\mathrm{H}(45)$ & 119.7 \\
\hline$C(35 B)-C(34 B)-H(34 E)$ & 108.0 & $\mathrm{C}(44)-\mathrm{C}(45)-\mathrm{H}(45)$ & 119.7 \\
\hline $\mathrm{C}(33 \mathrm{~B})-\mathrm{C}(34 \mathrm{~B})-\mathrm{H}(34 \mathrm{~F})$ & 108.0 & $C(41)-C(46)-C(45)$ & $118.07(16)$ \\
\hline
\end{tabular}




\begin{tabular}{|c|c|c|c|}
\hline $\mathrm{C}(41)-\mathrm{C}(46)-\mathrm{H}(46)$ & 121.0 & $\mathrm{C}(54)-\mathrm{C}(53)-\mathrm{C}(52)$ & $118.0(6)$ \\
\hline $\mathrm{C}(45)-\mathrm{C}(46)-\mathrm{H}(46)$ & 121.0 & $\mathrm{C}(54)-\mathrm{C}(53)-\mathrm{H}(53 \mathrm{~A})$ & 107.8 \\
\hline $\mathrm{O}(43)-\mathrm{C}(47)-\mathrm{O}(44)$ & $124.72(16)$ & $\mathrm{C}(52)-\mathrm{C}(53)-\mathrm{H}(53 \mathrm{~A})$ & 107.8 \\
\hline $\mathrm{O}(43)-\mathrm{C}(47)-\mathrm{C}(44)$ & $124.15(16)$ & $\mathrm{C}(54)-\mathrm{C}(53)-\mathrm{H}(53 \mathrm{~B})$ & 107.8 \\
\hline $\mathrm{O}(44)-\mathrm{C}(47)-\mathrm{C}(44)$ & $111.13(14)$ & $\mathrm{C}(52)-\mathrm{C}(53)-\mathrm{H}(53 \mathrm{~B})$ & 107.8 \\
\hline $\mathrm{O}(44)-\mathrm{C}(48)-\mathrm{C}(57)$ & $106.36(14)$ & $\mathrm{H}(53 \mathrm{~A})-\mathrm{C}(53)-\mathrm{H}(53 \mathrm{~B})$ & 107.2 \\
\hline $\mathrm{O}(44)-\mathrm{C}(48)-\mathrm{C}(49)$ & $109.79(15)$ & $\mathrm{C}(53)-\mathrm{C}(54)-\mathrm{C}(55)$ & $118.4(7)$ \\
\hline $\mathrm{C}(57)-\mathrm{C}(48)-\mathrm{C}(49)$ & $106.00(16)$ & $\mathrm{C}(53)-\mathrm{C}(54)-\mathrm{H}(54 \mathrm{~A})$ & 107.7 \\
\hline $\mathrm{O}(44)-\mathrm{C}(48)-\mathrm{H}(48)$ & 111.5 & $\mathrm{C}(55)-\mathrm{C}(54)-\mathrm{H}(54 \mathrm{~A})$ & 107.7 \\
\hline $\mathrm{C}(57)-\mathrm{C}(48)-\mathrm{H}(48)$ & 111.5 & $\mathrm{C}(53)-\mathrm{C}(54)-\mathrm{H}(54 \mathrm{~B})$ & 107.7 \\
\hline $\mathrm{C}(49)-\mathrm{C}(48)-\mathrm{H}(48)$ & 111.5 & $\mathrm{C}(55)-\mathrm{C}(54)-\mathrm{H}(54 \mathrm{~B})$ & 107.7 \\
\hline$C(50)-C(49)-C(48)$ & $106.18(16)$ & $\mathrm{H}(54 \mathrm{~A})-\mathrm{C}(54)-\mathrm{H}(54 \mathrm{~B})$ & 107.1 \\
\hline $\mathrm{C}(50)-\mathrm{C}(49)-\mathrm{H}(49 \mathrm{~A})$ & 110.5 & $C(56)-C(55)-C(54)$ & $112.1(8)$ \\
\hline $\mathrm{C}(48)-\mathrm{C}(49)-\mathrm{H}(49 \mathrm{~A})$ & 110.5 & $\mathrm{C}(56)-\mathrm{C}(55)-\mathrm{H}(55 \mathrm{~A})$ & 109.2 \\
\hline $\mathrm{C}(50)-\mathrm{C}(49)-\mathrm{H}(49 \mathrm{~B})$ & 110.5 & $\mathrm{C}(54)-\mathrm{C}(55)-\mathrm{H}(55 \mathrm{~A})$ & 109.2 \\
\hline C(48)-C(49)-H(49B) & 110.5 & $\mathrm{C}(56)-\mathrm{C}(55)-\mathrm{H}(55 \mathrm{~B})$ & 109.2 \\
\hline $\mathrm{H}(49 \mathrm{~A})-\mathrm{C}(49)-\mathrm{H}(49 \mathrm{~B})$ & 108.7 & $\mathrm{C}(54)-\mathrm{C}(55)-\mathrm{H}(55 \mathrm{~B})$ & 109.2 \\
\hline $\mathrm{C}(49)-\mathrm{C}(50)-\mathrm{C}(51)$ & $105.09(17)$ & $\mathrm{H}(55 \mathrm{~A})-\mathrm{C}(55)-\mathrm{H}(55 \mathrm{~B})$ & 107.9 \\
\hline $\mathrm{C}(49)-\mathrm{C}(50)-\mathrm{H}(50 \mathrm{~A})$ & 110.7 & $C(57)-C(56)-C(55)$ & $112.9(7)$ \\
\hline $\mathrm{C}(51)-\mathrm{C}(50)-\mathrm{H}(50 \mathrm{~A})$ & 110.7 & $\mathrm{C}(57)-\mathrm{C}(56)-\mathrm{H}(56 \mathrm{~A})$ & 109.0 \\
\hline $\mathrm{C}(49)-\mathrm{C}(50)-\mathrm{H}(50 \mathrm{~B})$ & 110.7 & $\mathrm{C}(55)-\mathrm{C}(56)-\mathrm{H}(56 \mathrm{~A})$ & 109.0 \\
\hline $\mathrm{C}(51)-\mathrm{C}(50)-\mathrm{H}(50 \mathrm{~B})$ & 110.7 & $\mathrm{C}(57)-\mathrm{C}(56)-\mathrm{H}(56 \mathrm{~B})$ & 109.0 \\
\hline $\mathrm{H}(50 \mathrm{~A})-\mathrm{C}(50)-\mathrm{H}(50 \mathrm{~B})$ & 108.8 & $\mathrm{C}(55)-\mathrm{C}(56)-\mathrm{H}(56 \mathrm{~B})$ & 109.0 \\
\hline $\mathrm{C}(57)-\mathrm{C}(51)-\mathrm{C}(50)$ & $102.25(18)$ & $\mathrm{H}(56 \mathrm{~A})-\mathrm{C}(56)-\mathrm{H}(56 \mathrm{~B})$ & 107.8 \\
\hline $\mathrm{C}(57)-\mathrm{C}(51)-\mathrm{C}(52)$ & $108.2(3)$ & $\mathrm{C}(53 \mathrm{~A})-\mathrm{C}(52 \mathrm{~A})-\mathrm{C}(51)$ & $116.1(10)$ \\
\hline $\mathrm{C}(50)-\mathrm{C}(51)-\mathrm{C}(52)$ & $111.6(3)$ & $\mathrm{C}(53 \mathrm{~A})-\mathrm{C}(52 \mathrm{~A})-\mathrm{H}(52 \mathrm{C})$ & 108.3 \\
\hline$C(57)-C(51)-C(52 A)$ & $123.7(4)$ & $\mathrm{C}(51)-\mathrm{C}(52 \mathrm{~A})-\mathrm{H}(52 \mathrm{C})$ & 108.3 \\
\hline$C(50)-C(51)-C(52 A)$ & $116.2(5)$ & $\mathrm{C}(53 \mathrm{~A})-\mathrm{C}(52 \mathrm{~A})-\mathrm{H}(52 \mathrm{D})$ & 108.3 \\
\hline $\mathrm{C}(57)-\mathrm{C}(51)-\mathrm{H}(51)$ & 111.5 & $\mathrm{C}(51)-\mathrm{C}(52 \mathrm{~A})-\mathrm{H}(52 \mathrm{D})$ & 108.3 \\
\hline $\mathrm{C}(50)-\mathrm{C}(51)-\mathrm{H}(51)$ & 111.5 & $\mathrm{H}(52 \mathrm{C})-\mathrm{C}(52 \mathrm{~A})-\mathrm{H}(52 \mathrm{D})$ & 107.4 \\
\hline $\mathrm{C}(52)-\mathrm{C}(51)-\mathrm{H}(51)$ & 111.5 & $\mathrm{C}(54 \mathrm{~A})-\mathrm{C}(53 \mathrm{~A})-\mathrm{C}(52 \mathrm{~A})$ & $114.8(11)$ \\
\hline $\mathrm{C}(57)-\mathrm{C}(51)-\mathrm{H}(51 \mathrm{~A})$ & 104.2 & $\mathrm{C}(54 \mathrm{~A})-\mathrm{C}(53 \mathrm{~A})-\mathrm{H}(53 \mathrm{C})$ & 108.6 \\
\hline $\mathrm{C}(50)-\mathrm{C}(51)-\mathrm{H}(51 \mathrm{~A})$ & 104.2 & $\mathrm{C}(52 \mathrm{~A})-\mathrm{C}(53 \mathrm{~A})-\mathrm{H}(53 \mathrm{C})$ & 108.6 \\
\hline $\mathrm{C}(52 \mathrm{~A})-\mathrm{C}(51)-\mathrm{H}(51 \mathrm{~A})$ & 104.2 & $\mathrm{C}(54 \mathrm{~A})-\mathrm{C}(53 \mathrm{~A})-\mathrm{H}(53 \mathrm{D})$ & 108.6 \\
\hline $\mathrm{C}(53)-\mathrm{C}(52)-\mathrm{C}(51)$ & $116.2(6)$ & $\mathrm{C}(52 \mathrm{~A})-\mathrm{C}(53 \mathrm{~A})-\mathrm{H}(53 \mathrm{D})$ & 108.6 \\
\hline $\mathrm{C}(53)-\mathrm{C}(52)-\mathrm{H}(52 \mathrm{~A})$ & 108.2 & $\mathrm{H}(53 \mathrm{C})-\mathrm{C}(53 \mathrm{~A})-\mathrm{H}(53 \mathrm{D})$ & 107.5 \\
\hline $\mathrm{C}(51)-\mathrm{C}(52)-\mathrm{H}(52 \mathrm{~A})$ & 108.2 & $C(53 \mathrm{~A})-\mathrm{C}(54 \mathrm{~A})-\mathrm{C}(55 \mathrm{~A})$ & $118.5(11)$ \\
\hline $\mathrm{C}(53)-\mathrm{C}(52)-\mathrm{H}(52 \mathrm{~B})$ & 108.2 & $\mathrm{C}(53 \mathrm{~A})-\mathrm{C}(54 \mathrm{~A})-\mathrm{H}(54 \mathrm{C})$ & 107.7 \\
\hline $\mathrm{C}(51)-\mathrm{C}(52)-\mathrm{H}(52 \mathrm{~B})$ & 108.2 & $\mathrm{C}(55 \mathrm{~A})-\mathrm{C}(54 \mathrm{~A})-\mathrm{H}(54 \mathrm{C})$ & 107.7 \\
\hline $\mathrm{H}(52 \mathrm{~A})-\mathrm{C}(52)-\mathrm{H}(52 \mathrm{~B})$ & 107.4 & $\mathrm{C}(53 \mathrm{~A})-\mathrm{C}(54 \mathrm{~A})-\mathrm{H}(54 \mathrm{D})$ & 107.7 \\
\hline
\end{tabular}




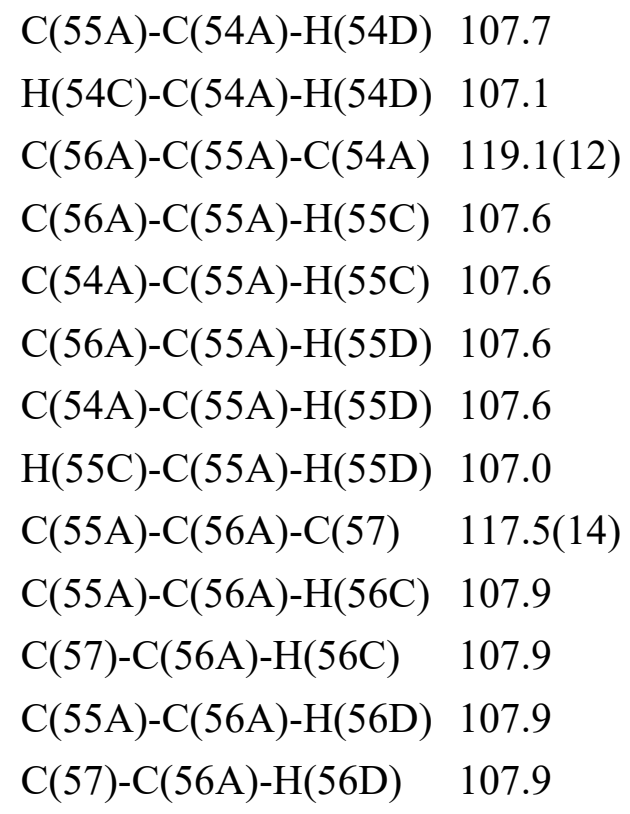

$\mathrm{H}(56 \mathrm{C})-\mathrm{C}(56 \mathrm{~A})-\mathrm{H}(56 \mathrm{D}) 107.2$

$\mathrm{C}(56)-\mathrm{C}(57)-\mathrm{C}(48) \quad 111.9(4)$

$\mathrm{C}(56)-\mathrm{C}(57)-\mathrm{C}(51) \quad 120.3(5)$

$\mathrm{C}(48)-\mathrm{C}(57)-\mathrm{C}(51) \quad 104.16(15)$

$\mathrm{C}(48)-\mathrm{C}(57)-\mathrm{C}(56 \mathrm{~A}) \quad 119.0(6)$

$\mathrm{C}(51)-\mathrm{C}(57)-\mathrm{C}(56 \mathrm{~A}) \quad 110.6(8)$

$\mathrm{C}(56)-\mathrm{C}(57)-\mathrm{H}(57) \quad 106.6$

$\mathrm{C}(48)-\mathrm{C}(57)-\mathrm{H}(57) \quad 106.6$

$\mathrm{C}(51)-\mathrm{C}(57)-\mathrm{H}(57) \quad 106.6$

$\mathrm{C}(48)-\mathrm{C}(57)-\mathrm{H}(57 \mathrm{~A}) \quad 107.5$

$\mathrm{C}(51)-\mathrm{C}(57)-\mathrm{H}(57 \mathrm{~A}) \quad 107.5$

C(56A)-C(57)-H(57A) 107.5 
Table S9. Anisotropic displacement parameters $\left(\AA^{2} \times 10^{3}\right)$ for 11. The anisotropic displacement factor exponent takes the form: $-2 \pi^{2}\left[\mathrm{~h}^{2} \mathrm{a}^{* 2} \mathrm{U}^{11}+\ldots+2 \mathrm{~h} \mathrm{k} \mathrm{a} \mathrm{b}^{*} \mathrm{U}^{12}\right]$

\begin{tabular}{|c|c|c|c|c|c|c|}
\hline & $\mathrm{U}^{11}$ & $\mathrm{U}^{22}$ & $\mathrm{U}^{33}$ & $\mathrm{U}^{23}$ & $\mathrm{U}^{13}$ & $\mathrm{U}^{12}$ \\
\hline $\mathrm{O}(1)$ & $34(1)$ & $25(1)$ & $46(1)$ & $-9(1)$ & $3(1)$ & $-5(1)$ \\
\hline $\mathrm{O}(2)$ & $19(1)$ & $36(1)$ & $48(1)$ & $-8(1)$ & $-1(1)$ & $-2(1)$ \\
\hline $\mathrm{O}(3)$ & $18(1)$ & $30(1)$ & $33(1)$ & $-10(1)$ & $0(1)$ & $-5(1)$ \\
\hline $\mathrm{O}(4)$ & $18(1)$ & $23(1)$ & $34(1)$ & $-5(1)$ & $1(1)$ & $-2(1)$ \\
\hline $\mathrm{N}(1)$ & $24(1)$ & $26(1)$ & $25(1)$ & $-9(1)$ & $1(1)$ & $-2(1)$ \\
\hline$C(1)$ & $20(1)$ & $26(1)$ & $18(1)$ & $-8(1)$ & $2(1)$ & $-2(1)$ \\
\hline$C(2)$ & $16(1)$ & $29(1)$ & $19(1)$ & $-9(1)$ & $2(1)$ & $-6(1)$ \\
\hline$C(3)$ & $22(1)$ & $26(1)$ & $18(1)$ & $-8(1)$ & $4(1)$ & $-7(1)$ \\
\hline$C(4)$ & $18(1)$ & $27(1)$ & $16(1)$ & $-7(1)$ & $2(1)$ & $-5(1)$ \\
\hline$C(5)$ & $19(1)$ & $30(1)$ & $20(1)$ & $-8(1)$ & $3(1)$ & $-7(1)$ \\
\hline$C(6)$ & $22(1)$ & $26(1)$ & $21(1)$ & $-8(1)$ & $4(1)$ & $-7(1)$ \\
\hline$C(7)$ & $22(1)$ & $27(1)$ & 19(1) & $-8(1)$ & $2(1)$ & $-6(1)$ \\
\hline$C(8)$ & $18(1)$ & $24(1)$ & $34(1)$ & $-8(1)$ & $1(1)$ & $2(1)$ \\
\hline $\mathrm{C}(9)$ & $27(1)$ & $28(1)$ & $34(1)$ & $-10(1)$ & $-3(1)$ & $2(1)$ \\
\hline$C(10)$ & $37(1)$ & $31(1)$ & $32(1)$ & $-6(1)$ & $-2(1)$ & $-1(1)$ \\
\hline $\mathrm{C}(11)$ & $26(1)$ & $23(1)$ & $31(1)$ & $-6(1)$ & $3(1)$ & $0(1)$ \\
\hline$C(12)$ & $32(1)$ & $26(1)$ & $39(1)$ & $-7(1)$ & $6(1)$ & $-2(1)$ \\
\hline$C(13)$ & $33(1)$ & $28(1)$ & $52(1)$ & $-10(1)$ & $8(1)$ & $-7(1)$ \\
\hline$C(14)$ & $31(1)$ & $35(1)$ & $52(1)$ & $-19(1)$ & $1(1)$ & $-7(1)$ \\
\hline$C(15)$ & $35(1)$ & $38(1)$ & $44(1)$ & $-20(1)$ & $2(1)$ & $-8(1)$ \\
\hline$C(15 A)$ & $30(7)$ & $33(8)$ & $36(11)$ & $-11(8)$ & $-7(7)$ & $-8(7)$ \\
\hline$C(16)$ & $33(1)$ & $40(1)$ & $34(1)$ & $-12(1)$ & $3(1)$ & $-10(1)$ \\
\hline$C(17)$ & $22(1)$ & $23(1)$ & $33(1)$ & $-9(1)$ & $3(1)$ & $1(1)$ \\
\hline $\mathrm{O}(21)$ & $33(1)$ & $27(1)$ & $62(1)$ & $-11(1)$ & $5(1)$ & $-6(1)$ \\
\hline $\mathrm{O}(22)$ & $20(1)$ & $38(1)$ & $40(1)$ & $-15(1)$ & $4(1)$ & $-3(1)$ \\
\hline $\mathrm{O}(23)$ & $23(1)$ & $39(1)$ & $48(1)$ & $-9(1)$ & $-3(1)$ & $-3(1)$ \\
\hline $\mathrm{N}(21)$ & $24(1)$ & $30(1)$ & $30(1)$ & $-13(1)$ & $6(1)$ & $-4(1)$ \\
\hline $\mathrm{C}(21)$ & $21(1)$ & $30(1)$ & $19(1)$ & $-12(1)$ & $5(1)$ & $-3(1)$ \\
\hline $\mathrm{C}(22)$ & $25(1)$ & $28(1)$ & $24(1)$ & $-12(1)$ & $6(1)$ & $-9(1)$ \\
\hline$C(23)$ & $20(1)$ & $34(1)$ & $23(1)$ & $-12(1)$ & $3(1)$ & $-8(1)$ \\
\hline $\mathrm{C}(24)$ & $23(1)$ & $31(1)$ & $17(1)$ & $-9(1)$ & $2(1)$ & $-5(1)$ \\
\hline$C(25)$ & $26(1)$ & $29(1)$ & $20(1)$ & $-11(1)$ & $4(1)$ & $-8(1)$ \\
\hline$C(26)$ & $20(1)$ & $31(1)$ & $23(1)$ & $-13(1)$ & $4(1)$ & $-7(1)$ \\
\hline$C(27)$ & $25(1)$ & $34(1)$ & $26(1)$ & $-9(1)$ & $-1(1)$ & $-5(1)$ \\
\hline $\mathrm{O}(24)$ & $27(4)$ & $22(2)$ & $51(7)$ & $-10(3)$ & $-7(4)$ & $-2(2)$ \\
\hline
\end{tabular}




\begin{tabular}{|c|c|c|c|c|c|c|}
\hline$C(28)$ & $25(3)$ & $26(3)$ & $46(4)$ & $-11(2)$ & $-16(2)$ & $1(2)$ \\
\hline$C(29)$ & $29(3)$ & $27(3)$ & $51(3)$ & $-10(2)$ & $1(2)$ & $-5(2)$ \\
\hline$C(30)$ & $40(3)$ & $27(2)$ & $47(3)$ & $-14(2)$ & $1(2)$ & $-9(2)$ \\
\hline $\mathrm{C}(31)$ & $30(3)$ & $33(3)$ & $37(3)$ & $-16(2)$ & $-4(2)$ & $-6(2)$ \\
\hline$C(32)$ & $51(3)$ & $38(3)$ & $64(3)$ & $-28(3)$ & $7(2)$ & $-15(2)$ \\
\hline$C(33)$ & $61(4)$ & $45(4)$ & $100(7)$ & $-34(4)$ & $22(4)$ & $-21(3)$ \\
\hline C(34) & $48(2)$ & $79(2)$ & $73(3)$ & $-30(2)$ & $5(2)$ & $-6(2)$ \\
\hline$C(35)$ & $49(2)$ & $46(2)$ & $44(2)$ & $-7(2)$ & $4(2)$ & $4(2)$ \\
\hline$C(36)$ & $58(2)$ & $34(3)$ & $38(2)$ & $-12(2)$ & $0(2)$ & $3(2)$ \\
\hline$C(37)$ & $31(3)$ & $22(2)$ & $35(3)$ & $-8(2)$ & $-6(2)$ & $1(2)$ \\
\hline $\mathrm{O}(24 \mathrm{~A})$ & $30(8)$ & $30(5)$ & $30(6)$ & $-9(5)$ & $-10(5)$ & $-3(4)$ \\
\hline$C(28 \mathrm{~A})$ & $37(7)$ & $30(6)$ & $43(7)$ & $-10(5)$ & $-9(6)$ & $2(4)$ \\
\hline$C(29 A)$ & $50(8)$ & $33(7)$ & $59(8)$ & $-8(6)$ & $8(7)$ & $-2(5)$ \\
\hline$C(30 A)$ & $55(8)$ & $73(10)$ & $50(6)$ & $-37(6)$ & $5(6)$ & $-12(6)$ \\
\hline$C(31 \mathrm{~A})$ & $47(8)$ & $27(5)$ & $48(6)$ & $-21(5)$ & $-13(6)$ & $0(5)$ \\
\hline$C(32 A)$ & $51(3)$ & $38(3)$ & $64(3)$ & $-28(3)$ & $7(2)$ & $-15(2)$ \\
\hline$C(33 A)$ & $48(6)$ & $38(7)$ & $50(6)$ & $-21(5)$ & $-1(5)$ & $-22(5)$ \\
\hline$C(34 A)$ & $48(2)$ & $79(2)$ & $73(3)$ & $-30(2)$ & $5(2)$ & $-6(2)$ \\
\hline$C(35 A)$ & $53(4)$ & $41(4)$ & $36(4)$ & $-21(4)$ & $1(4)$ & $0(4)$ \\
\hline$C(36 A)$ & $58(2)$ & $34(3)$ & $38(2)$ & $-12(2)$ & $0(2)$ & $3(2)$ \\
\hline$C(37 A)$ & $35(6)$ & $44(6)$ & $33(6)$ & $-22(5)$ & $-10(4)$ & $-7(5)$ \\
\hline $\mathrm{O}(24 \mathrm{~B})$ & $22(9)$ & $31(5)$ & $45(13)$ & $-10(7)$ & $-8(9)$ & $-2(5)$ \\
\hline$C(28 B)$ & $46(9)$ & $25(7)$ & $48(7)$ & $-11(6)$ & $-13(7)$ & $4(6)$ \\
\hline$C(29 B)$ & $77(11)$ & $36(8)$ & $52(8)$ & $-22(6)$ & $-19(7)$ & $2(7)$ \\
\hline $\mathrm{C}(30 \mathrm{~B})$ & $82(11)$ & $51(9)$ & $50(7)$ & $-9(7)$ & $-15(8)$ & $-5(8)$ \\
\hline$C(31 B)$ & $68(9)$ & $32(7)$ & $41(7)$ & $-20(6)$ & $6(6)$ & $-9(7)$ \\
\hline$C(32 B)$ & $70(9)$ & $54(8)$ & $60(7)$ & $-27(7)$ & $12(7)$ & $-21(7)$ \\
\hline$C(33 B)$ & $51(3)$ & $38(3)$ & $64(3)$ & $-28(3)$ & $7(2)$ & $-15(2)$ \\
\hline $\mathrm{C}(34 \mathrm{~B})$ & $60(10)$ & $58(8)$ & $56(9)$ & $-40(7)$ & $6(7)$ & $-20(7)$ \\
\hline$C(35 B)$ & $58(10)$ & $46(8)$ & $58(9)$ & $-28(7)$ & $-5(7)$ & $-11(7)$ \\
\hline$C(36 B)$ & $42(9)$ & $45(8)$ & $53(7)$ & $-32(7)$ & $7(7)$ & $-11(7)$ \\
\hline$C(37 B)$ & $46(8)$ & $28(6)$ & $49(6)$ & $-10(6)$ & $-7(6)$ & $-5(6)$ \\
\hline $\mathrm{O}(41)$ & $25(1)$ & $35(1)$ & $44(1)$ & $-16(1)$ & $3(1)$ & $-10(1)$ \\
\hline $\mathrm{O}(42)$ & $36(1)$ & $26(1)$ & $90(1)$ & $-23(1)$ & $-2(1)$ & $-1(1)$ \\
\hline $\mathrm{O}(43)$ & $20(1)$ & $37(1)$ & $43(1)$ & $-18(1)$ & $4(1)$ & $-8(1)$ \\
\hline $\mathrm{O}(44)$ & $24(1)$ & $23(1)$ & $37(1)$ & $-11(1)$ & $1(1)$ & $-6(1)$ \\
\hline $\mathrm{N}(41)$ & $26(1)$ & $27(1)$ & $31(1)$ & $-12(1)$ & $-2(1)$ & $-4(1)$ \\
\hline$C(41)$ & $23(1)$ & $24(1)$ & $18(1)$ & $-9(1)$ & $1(1)$ & $-5(1)$ \\
\hline$C(42)$ & $17(1)$ & $29(1)$ & $19(1)$ & $-12(1)$ & $1(1)$ & $-2(1)$ \\
\hline$C(43)$ & $22(1)$ & $24(1)$ & $20(1)$ & $-10(1)$ & $1(1)$ & $-2(1)$ \\
\hline
\end{tabular}




$\begin{array}{lllllll}\mathrm{C}(44) & 20(1) & 26(1) & 19(1) & -10(1) & 2(1) & -3(1) \\ \mathrm{C}(45) & 18(1) & 30(1) & 26(1) & -11(1) & 2(1) & -1(1) \\ \mathrm{C}(46) & 23(1) & 23(1) & 24(1) & -8(1) & -1(1) & 0(1) \\ \mathrm{C}(47) & 21(1) & 29(1) & 24(1) & -13(1) & 3(1) & -4(1) \\ \mathrm{C}(48) & 29(1) & 28(1) & 43(1) & -15(1) & 4(1) & -14(1) \\ \mathrm{C}(49) & 36(1) & 32(1) & 48(1) & -16(1) & 13(1) & -12(1) \\ \mathrm{C}(50) & 70(2) & 31(1) & 50(1) & -4(1) & 26(1) & 0(1) \\ \mathrm{C}(51) & 50(1) & 24(1) & 35(1) & -4(1) & 9(1) & 4(1) \\ \mathrm{C}(52) & 67(4) & 24(2) & 59(5) & 9(2) & 32(3) & 10(2) \\ \mathrm{C}(53) & 54(4) & 39(4) & 45(3) & 4(2) & 9(3) & 15(3) \\ \mathrm{C}(54) & 26(2) & 40(2) & 41(2) & -9(2) & 2(1) & -2(2) \\ \mathrm{C}(55) & 39(4) & 34(4) & 31(3) & -14(2) & 3(2) & -10(3) \\ \mathrm{C}(56) & 42(3) & 27(3) & 34(3) & -16(2) & 0(2) & 1(2) \\ \mathrm{C}(52 \mathrm{~A}) & 87(7) & 35(5) & 25(4) & -14(3) & -9(4) & 10(4) \\ \mathrm{C}(53 \mathrm{~A}) & 42(5) & 34(6) & 55(6) & -14(5) & -26(4) & 10(4) \\ \mathrm{C}(54 \mathrm{~A}) & 62(4) & 45(4) & 41(4) & -19(3) & -5(3) & 21(4) \\ \mathrm{C}(55 \mathrm{~A}) & 36(7) & 29(6) & 59(7) & -21(4) & -3(5) & 14(6) \\ \mathrm{C}(56 \mathrm{~A}) & 41(6) & 33(5) & 25(5) & -6(4) & 6(4) & 3(4) \\ \mathrm{C}(57) & 39(1) & 26(1) & 46(1) & -19(1) & 9(1) & -14(1)\end{array}$


Table S10. Hydrogen coordinates $\left(\mathrm{x} 10^{4}\right)$ and isotropic displacement parameters $\left(\AA^{2} \times 10^{3}\right)$ for 11.

\begin{tabular}{|c|c|c|c|c|}
\hline & $\mathrm{x}$ & $\mathrm{y}$ & $\mathrm{z}$ & $\mathrm{U}(\mathrm{eq})$ \\
\hline $\mathrm{H}(2)$ & 9794 & 5090 & 9289 & 25 \\
\hline $\mathrm{H}(3)$ & 7374 & 6177 & 8867 & 26 \\
\hline $\mathrm{H}(5)$ & 3214 & 4785 & 9095 & 27 \\
\hline $\mathrm{H}(6)$ & 5618 & 3694 & 9538 & 27 \\
\hline $\mathrm{H}(8)$ & 1518 & 7572 & 8589 & 32 \\
\hline $\mathrm{H}(9 \mathrm{~A})$ & 716 & 7995 & 7378 & 37 \\
\hline $\mathrm{H}(9 \mathrm{AB})$ & 2483 & 7351 & 7321 & 37 \\
\hline $\mathrm{H}(10 \mathrm{~A})$ & 4000 & 8329 & 6601 & 42 \\
\hline $\mathrm{H}(10 \mathrm{~B})$ & 2478 & 8953 & 6816 & 42 \\
\hline $\mathrm{H}(11)$ & 6018 & 8075 & 7591 & 34 \\
\hline $\mathrm{H}(12 \mathrm{~A})$ & 4579 & 9641 & 7411 & 41 \\
\hline $\mathrm{H}(12 \mathrm{~B})$ & 5789 & 9447 & 6767 & 41 \\
\hline $\mathrm{H}(13 \mathrm{~A})$ & 8644 & 9129 & 7383 & 47 \\
\hline $\mathrm{H}(13 \mathrm{~B})$ & 7601 & 9831 & 7610 & 47 \\
\hline $\mathrm{H}(14 \mathrm{~A})$ & 8904 & 8972 & 8675 & 45 \\
\hline $\mathrm{H}(14 \mathrm{~B})$ & 8339 & 8254 & 8507 & 45 \\
\hline $\mathrm{H}(14 \mathrm{C})$ & 9290 & 8689 & 8572 & 45 \\
\hline $\mathrm{H}(14 \mathrm{D})$ & 7268 & 9108 & 8762 & 45 \\
\hline $\mathrm{H}(15 \mathrm{~A})$ & 5207 & 9279 & 8784 & 45 \\
\hline $\mathrm{H}(15 \mathrm{~B})$ & 6502 & 8687 & 9474 & 45 \\
\hline $\mathrm{H}(15 \mathrm{C})$ & 7876 & 7702 & 9246 & 39 \\
\hline $\mathrm{H}(15 \mathrm{D})$ & 7293 & 7756 & 8437 & 39 \\
\hline $\mathrm{H}(16 \mathrm{~A})$ & 5763 & 7640 & 9108 & 42 \\
\hline$H(16 B)$ & 3897 & 8069 & 9393 & 42 \\
\hline $\mathrm{H}(16 \mathrm{C})$ & 4521 & 7661 & 9381 & 42 \\
\hline $\mathrm{H}(16 \mathrm{D})$ & 4531 & 8574 & 9160 & 42 \\
\hline $\mathrm{H}(17)$ & 2694 & 8728 & 8221 & 32 \\
\hline $\mathrm{H}(22)$ & 10017 & 6273 & 7219 & 29 \\
\hline $\mathrm{H}(23)$ & 12457 & 5194 & 7621 & 30 \\
\hline $\mathrm{H}(25)$ & 8368 & 3781 & 7825 & 29 \\
\hline $\mathrm{H}(26)$ & 5905 & 4858 & 7427 & 28 \\
\hline $\mathrm{H}(28)$ & 14130 & 2374 & 8630 & 40 \\
\hline $\mathrm{H}(29 \mathrm{~A})$ & 13654 & 2590 & 7177 & 44 \\
\hline $\mathrm{H}(29 \mathrm{~B})$ & 15429 & 2016 & 7693 & 44 \\
\hline $\mathrm{H}(30 \mathrm{~A})$ & 12698 & 1515 & 7123 & 45 \\
\hline $\mathrm{H}(30 \mathrm{~B})$ & 14007 & 969 & 7835 & 45 \\
\hline
\end{tabular}


$\mathrm{H}(31)$

$\mathrm{H}(32 \mathrm{~A})$

$\mathrm{H}(32 \mathrm{~B})$

$\mathrm{H}(33 \mathrm{~A})$

$\mathrm{H}(33 \mathrm{~B})$

$\mathrm{H}(34 \mathrm{~A})$

$\mathrm{H}(34 \mathrm{~B})$

$\mathrm{H}(35 \mathrm{~A})$

$\mathrm{H}(35 \mathrm{~B})$

$\mathrm{H}(36 \mathrm{~A})$

$\mathrm{H}(36 \mathrm{~B})$

$\mathrm{H}(37)$

$\mathrm{H}(28 \mathrm{~A})$

$\mathrm{H}(29 \mathrm{C})$

H(29D)

$\mathrm{H}(30 \mathrm{C})$

$\mathrm{H}(30 \mathrm{D})$

$\mathrm{H}(31 \mathrm{~A})$

$\mathrm{H}(32 \mathrm{C})$

H(32D)

$\mathrm{H}(33 \mathrm{C})$

H(33D)

$\mathrm{H}(34 \mathrm{C})$

H(34D)

$\mathrm{H}(35 \mathrm{C})$

$\mathrm{H}(35 \mathrm{D})$

$\mathrm{H}(36 \mathrm{C})$

$\mathrm{H}(36 \mathrm{D})$

$\mathrm{H}(37 \mathrm{~A})$

$\mathrm{H}(28 \mathrm{~B})$

$\mathrm{H}(29 \mathrm{E})$

H(29F)

$\mathrm{H}(30 \mathrm{E})$

$\mathrm{H}(30 \mathrm{~F})$

H(31B)

H(32E)

$\mathrm{H}(32 \mathrm{~F})$

H(33E)

$\mathrm{H}(33 \mathrm{~F})$

H(34E)
10141

10607

11491

7556

8282

6944

7161

10455

8848

11129

9434

12951

14118

13913

15658

14426

13130

10528

10958

11497

7783

8144

8612

6507

8247

7565

10699

10867

13200

14704

14801

12950

11211

12905

9826

11142

8958

8712

8699

10672
1813

408

263

849

78

737

1542

686

1242

2022

2275

1236

2461

2722

2145

1109

1684

1895

498

338

1121

240

608

998

2151

2032

2225

1308

1260

2512

2007

2661

1697

1073

2049

390

800

309

1209

264
$7823 \quad 39$

$8003 \quad 57$

$8798 \quad 57$

$8294 \quad 78$

$8963 \quad 78$

$9591 \quad 80$

$8962 \quad 80$

$9876 \quad 60$

$10143 \quad 60$

$9591 \quad 53$

$8975 \quad 53$

$9011 \quad 36$

$8755 \quad 46$

$7291 \quad 60$

$7820 \quad 60$

$7839 \quad 66$

$7134 \quad 66$

$7832 \quad 47$

$7952 \quad 57$

$8790 \quad 57$

$8103 \quad 50$

$8638 \quad 50$

$9634 \quad 80$

$9253 \quad 80$

$8572 \quad 50$

$9398 \quad 50$

$9432 \quad 53$

$9781 \quad 53$

$9051 \quad 41$

$8192 \quad 49$

$9457 \quad 64$

$9405 \quad 64$

$9941 \quad 76$

$9801 \quad 76$

$8768 \quad 54$

$9298 \quad 70$

$9355 \quad 70$

$8455 \quad 57$

$8022 \quad 57$

7570

76

70

39

7

(1)

80

80

(2)

3

53

36

46

60

60

66

66

47

57

57

50

50

80

80

50

0

53

53

41

49

64

64

76




\begin{tabular}{|c|c|c|c|}
\hline $\mathrm{H}(34 \mathrm{~F})$ & 12243 & 205 & 8177 \\
\hline$H(35 E)$ & 11325 & 1385 & 6828 \\
\hline $\mathrm{H}(35 \mathrm{~F})$ & 13420 & 1058 & 7233 \\
\hline $\mathrm{H}(36 \mathrm{E})$ & 12743 & 2297 & 7199 \\
\hline $\mathrm{H}(36 \mathrm{~F})$ & 10527 & 2207 & 7453 \\
\hline $\mathrm{H}(37 \mathrm{~B})$ & 13543 & 1321 & 8451 \\
\hline $\mathrm{H}(42)$ & -1952 & 5037 & 5888 \\
\hline $\mathrm{H}(43)$ & -316 & 6116 & 5568 \\
\hline $\mathrm{H}(45)$ & 4926 & 4735 & 5793 \\
\hline $\mathrm{H}(46)$ & 3302 & 3652 & 6090 \\
\hline $\mathrm{H}(48)$ & 4367 & 7511 & 5394 \\
\hline $\mathrm{H}(49 \mathrm{~A})$ & 5334 & 7905 & 4216 \\
\hline H(49B) & 3895 & 7373 & 4062 \\
\hline $\mathrm{H}(50 \mathrm{~A})$ & 2223 & 8496 & 3326 \\
\hline $\mathrm{H}(50 \mathrm{~B})$ & 3186 & 8989 & 3719 \\
\hline $\mathrm{H}(51)$ & -176 & 8182 & 4218 \\
\hline $\mathrm{H}(51 \mathrm{~A})$ & 82 & 8093 & 4252 \\
\hline $\mathrm{H}(52 \mathrm{~A})$ & 484 & 9610 & 4356 \\
\hline $\mathrm{H}(52 \mathrm{~B})$ & -378 & 9628 & 3598 \\
\hline $\mathrm{H}(53 \mathrm{~A})$ & -2742 & 9939 & 4450 \\
\hline $\mathrm{H}(53 \mathrm{~B})$ & -3309 & 9371 & 4069 \\
\hline $\mathrm{H}(54 \mathrm{~A})$ & -3100 & 8368 & 5110 \\
\hline $\mathrm{H}(54 \mathrm{~B})$ & -4029 & 9062 & 5367 \\
\hline $\mathrm{H}(55 \mathrm{~A})$ & -589 & 9046 & 5745 \\
\hline $\mathrm{H}(55 \mathrm{~B})$ & -1862 & 8420 & 6270 \\
\hline $\mathrm{H}(56 \mathrm{~A})$ & 1149 & 7739 & 6177 \\
\hline $\mathrm{H}(56 \mathrm{~B})$ & -348 & 7526 & 5695 \\
\hline $\mathrm{H}(52 \mathrm{C})$ & -222 & 9700 & 3943 \\
\hline $\mathrm{H}(52 \mathrm{D})$ & -1285 & 9286 & 3504 \\
\hline $\mathrm{H}(53 \mathrm{C})$ & -3665 & 9664 & 4187 \\
\hline $\mathrm{H}(53 \mathrm{D})$ & -3157 & 8751 & 4590 \\
\hline $\mathrm{H}(54 \mathrm{C})$ & -3572 & 9522 & 5371 \\
\hline $\mathrm{H}(54 \mathrm{D})$ & -1510 & 9793 & 5075 \\
\hline $\mathrm{H}(55 \mathrm{C})$ & -325 & 8889 & 6062 \\
\hline $\mathrm{H}(55 \mathrm{D})$ & -2223 & 8466 & 6183 \\
\hline $\mathrm{H}(56 \mathrm{C})$ & -953 & 7750 & 5483 \\
\hline $\mathrm{H}(56 \mathrm{D})$ & 593 & 7644 & 6115 \\
\hline $\mathrm{H}(57)$ & 2280 & 8631 & 5115 \\
\hline $\mathrm{H}(57 \mathrm{~A})$ & 2256 & 8630 & 5128 \\
\hline
\end{tabular}




\section{Geometries and Energies}

\section{Cycloheptane}

Geometries were optimized at MP2/6-311+G(d,p) and single point energies were computed at $\operatorname{CCSD}(\mathrm{T}) /$ aug-cc-pVTZ.

TC conformation ( $C_{2}$ point group)

$$
\begin{array}{rrr}
-0.319390000 & 2.586867000 & -0.518677000 \\
0.00000000 & 1.560443000 & -0.301068000 \\
1.082763000 & 1.605848000 & -0.119142000 \\
-0.735844000 & 1.071662000 & 0.956231000 \\
-0.941655000 & 1.920974000 & 1.618854000 \\
-1.716683000 & 0.688313000 & 0.646112000 \\
0.000000000 & 0.000000000 & 1.775549000 \\
-0.728927000 & -0.488797000 & 2.434457000 \\
0.728927000 & 0.488797000 & 2.434457000 \\
0.735844000 & -1.071662000 & 0.956231000 \\
1.716683000 & -0.688313000 & 0.646112000 \\
0.941655000 & -1.920974000 & 1.618854000 \\
0.000000000 & -1.560443000 & -0.301068000 \\
0.319390000 & -2.586867000 & -0.518677000 \\
-1.082763000 & -1.605848000 & -0.119142000 \\
0.298270000 & -0.705299000 & -1.540147000 \\
1.390223000 & -0.632914000 & -1.643503000 \\
-0.063275000 & -1.228433000 & -2.434845000 \\
-0.298270000 & 0.705299000 & -1.540147000 \\
0.063275000 & 1.228433000 & -2.434845000 \\
-1.390223000 & 0.632914000 & -1.643503000
\end{array}
$$

\section{C conformation ( $C_{\mathrm{s}}$ point group)}

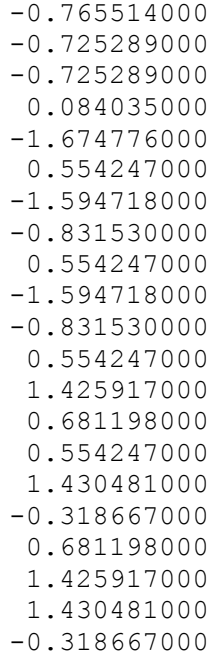

\footnotetext{
$\boldsymbol{E}[\mathrm{MP} 2]=-274.354738$ a.u.

$\boldsymbol{Z P V E}[\mathrm{MP} 2]=126.05827 \mathrm{kcal} \mathrm{mol}^{-1}$

$\boldsymbol{E}[\operatorname{CCSD}(\mathrm{T})]=-274.683426$ a.u.
} 
B conformation ( $C_{\mathrm{s}}$ point group)

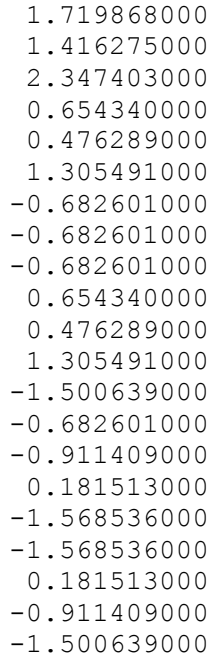

\section{$\boldsymbol{E}[\mathrm{MP} 2]=-274.351785$ a.u. \\ $\boldsymbol{Z P V E}[\mathrm{MP} 2]=126.28681 \mathrm{kcal} \mathrm{mol}^{-1}$ \\ $\boldsymbol{E}[\operatorname{CCSD}(\mathrm{T})]=-274.680482$ a.u.}

\section{TB conformation ( $C_{2}$ point group)}

$$
\begin{array}{rrr}
0.000000000 & 1.282890000 & 0.870668000 \\
0.714207000 & 0.279580000 & -1.431855000 \\
-0.714207000 & -0.279580000 & -1.431855000 \\
-1.004074000 & -1.292325000 & -0.289973000 \\
-0.893125000 & -0.773859000 & -2.393930000 \\
1.422233000 & -0.555393000 & -1.391290000 \\
0.000000000 & -1.282890000 & 0.870668000 \\
-2.012746000 & -1.122812000 & 0.108581000 \\
-1.009767000 & -2.304488000 & -0.712084000 \\
0.000000000 & 0.000000000 & 1.709289000 \\
-0.214754000 & -2.135349000 & 1.527189000 \\
1.006729000 & -1.456769000 & 0.474752000 \\
-0.880001000 & 0.007853000 & 2.365875000 \\
0.880001000 & -0.007853000 & 2.365875000 \\
1.004074000 & 1.292325000 & -0.289973000 \\
-1.422233000 & 0.555393000 & -1.391290000 \\
0.893125000 & 0.773859000 & -2.393930000 \\
1.009767000 & 2.304488000 & -0.712084000 \\
2.012746000 & 1.122812000 & 0.108581000 \\
0.214754000 & 2.135349000 & 1.527189000 \\
-1.006729000 & 1.456769000 & 0.474752000 \\
& &
\end{array}
$$

\section{T3 conformation ( $C_{2}$ point group)}

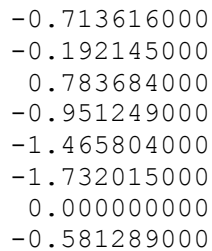

2. 509915000

1. 580234000

1.885907000

0.846980000

1. 567686000

0.215288000

0.000000000

$-0.663146000$
$-0.471844000$

$-0.217136000$

0.180905000

0.892328000

1. 538652000

0.450966000

1. 746423000

2.400184000 


$$
\begin{array}{rrr}
0.581289000 & 0.663146000 & 2.400184000 \\
0.951249000 & -0.846980000 & 0.892328000 \\
1.732015000 & -0.215288000 & 0.450966000 \\
1.465804000 & -1.567686000 & 1.538652000 \\
0.192145000 & -1.580234000 & -0.217136000 \\
0.713616000 & -2.509915000 & -0.471844000 \\
-0.783684000 & -1.885907000 & 0.180905000 \\
0.000000000 & -0.780861000 & -1.529805000 \\
0.792271000 & -1.087512000 & -2.221897000 \\
-0.933229000 & -1.126823000 & -1.988555000 \\
0.000000000 & 0.780861000 & -1.529805000 \\
0.933229000 & 1.126823000 & -1.988555000 \\
-0.792271000 & 1.087512000 & -2.221897000 \\
& & \\
\boldsymbol{E}[\mathrm{MP} 2]=-274.343545 \mathrm{a} . \mathrm{u} . \\
\boldsymbol{Z P V} \boldsymbol{E}[\mathrm{MP} 2]=126.24975 \mathrm{kcal} \mathrm{mol}{ }^{-1} \\
\boldsymbol{E}[\mathrm{CCSD}(\mathrm{T})]=-274.672482 \text { a.u. }
\end{array}
$$



2. 534130000
$-0.172664000$
2.539270000
1. 828503000
1.663922000
3. 621398000
1. 721835000
2.203045000
$-0.589976000$

\section{$\boldsymbol{E}$ [MP2] $=-391.0175167$ a.u. \\ $\boldsymbol{Z P V E}[\mathrm{MP} 2]=166.74058 \mathrm{kcal} \mathrm{mol}^{-1}$ \\ $\boldsymbol{E}[\operatorname{CCSD}(\mathrm{T})]=-391.1827093$ a.u.}

$-0.582122000$

0.251352000

$-1.262776000$

$-1.533840000$

\section{Conformer 1c-III}

$$
\begin{array}{rrr}
0.484507000 & -0.784869000 & -0.302970000 \\
-2.006885000 & -1.291616000 & 0.072081000 \\
-2.279934000 & 0.000005000 & -0.694612000 \\
-2.006883000 & 1.291614000 & 0.072100000 \\
-3.321210000 & 0.000008000 & -1.023655000 \\
-2.706110000 & -1.379233000 & 0.907034000 \\
-0.568423000 & 1.431897000 & 0.590432000 \\
-2.223234000 & 2.127531000 & -0.596842000 \\
-2.706097000 & 1.379211000 & 0.907065000 \\
0.484508000 & 0.784867000 & -0.302975000 \\
-0.330594000 & 2.493292000 & 0.695861000 \\
-0.481403000 & 1.011791000 & 1.593627000 \\
0.320534000 & 1.127932000 & -1.328560000 \\
1.911785000 & 1.181293000 & 0.111731000 \\
-0.568433000 & -1.431894000 & 0.590432000 \\
-1.679551000 & 0.000011000 & -1.606931000 \\
-2.223218000 & -2.127524000 & -0.596878000 \\
-0.330604000 & -2.493287000 & 0.695873000 \\
-0.481426000 & -1.011778000 & 1.593624000 \\
1.911780000 & -1.181293000 & 0.111751000 \\
0.320542000 & -1.127941000 & -1.328554000 \\
1.956538000 & 1.279531000 & 1.200548000 \\
2.217542000 & 2.135387000 & -0.317068000 \\
2.775878000 & -0.000005000 & -0.316606000 \\
3.767117000 & -0.000003000 & 0.135197000 \\
2.899702000 & -0.000015000 & -1.402069000 \\
2.217535000 & -2.135397000 & -0.317029000 \\
1.956528000 & -1.279510000 & 1.200569000 \\
& & \\
\boldsymbol{Z} \boldsymbol{E} \boldsymbol{E}[\mathrm{VE}[\mathrm{MP} 2]=-391.017352 \mathrm{a} 2 \mathrm{u} . & \\
\boldsymbol{E}[\mathrm{CCSD}(\mathrm{T})]=-166.84991 \mathrm{kcal} \mathrm{mol}-1
\end{array}
$$

\section{$\boldsymbol{E}[\mathrm{MP} 2]=-391.017352$ a.u. \\ $\boldsymbol{Z P V E}[\mathrm{MP} 2]=166.84991 \mathrm{kcal} \mathrm{mol}^{-1}$ \\ $\boldsymbol{E}[\mathrm{CCSD}(\mathrm{T})]=-391.1823432$ a.u.}

\section{Conformer 1c-IV}



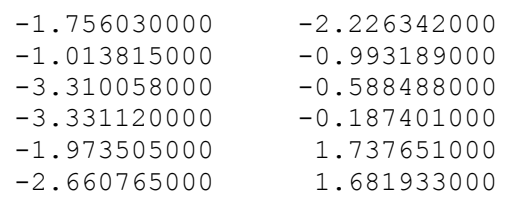

$-0.444159000$

$-1.463836000$

0.490797000

$-1.220897000$

$-0.887935000$

0.721130000

$$
\begin{gathered}
\boldsymbol{E}[\mathrm{MP} 2]=-391.0147255 \text { a.u. } \\
\boldsymbol{Z P \boldsymbol { E } E}[\mathrm{MP} 2]=166.78526 \mathrm{kcal} \mathrm{mol}^{-1} \\
\boldsymbol{E}[\mathrm{CCSD}(\mathrm{T})]=-391.1799221 \mathrm{a.u} .
\end{gathered}
$$

\section{Conformer 1c-V}

$$
\begin{aligned}
& -1.958052000 \\
& -1.958051000 \\
& -1.286258000 \\
& 1.286257000 \\
& 1.419919000 \\
& 0.787008000 \\
& 2.481872000 \\
& 2.126927000 \\
& -0.787010000 \\
& 1.180309000 \\
& 1.151075000 \\
& -1.419915000 \\
& -1.180311000 \\
& -1.151080000 \\
& -1.002014000 \\
& -2.481867000 \\
& 0.000001000 \\
& 1.002027000 \\
& 1.374043000 \\
& 0.000002000 \\
& 0.000004000 \\
& -2.126925000 \\
& -1.374053000 \\
& -1.274557000 \\
& 0.000000000 \\
& -2.132987000 \\
& 0.000001000 \\
& -0.000001000 \\
& 1.274551000 \\
& -0.195226000 \\
& -0.195233000 \\
& -0.518668000 \\
& 0.518792000 \\
& -0.590277000 \\
& 0.435638000 \\
& 0.518789000 \\
& 0.247717000 \\
& \text { 1. } 503009000 \\
& -0.518671000 \\
& 0.247714000 \\
& 1.503005000 \\
& -1.502528000 \\
& -0.590292000 \\
& 0.530922000 \\
& -1.502527000 \\
& -1.116139000 \\
& 0.713967000 \\
& 1.516154000 \\
& 0.435653000 \\
& -1.116127000 \\
& 1.189548000 \\
& -0.532287000 \\
& -0.275930000 \\
& -1.554563000 \\
& -0.585317000 \\
& 1.189551000 \\
& -0.275924000 \\
& \boldsymbol{Z P V E}[\mathrm{MP} 2]=166.91110 \mathrm{kcal} \mathrm{mol}^{-1} \\
& \boldsymbol{E}[\operatorname{CCSD}(\mathrm{T})]=-391.1796292 \text { a.u. }
\end{aligned}
$$

\section{Conformer 1c-VI}

$\begin{array}{lr}1 & -1.133677000 \\ 6 & -1.058668000 \\ 1 & -1.380580000 \\ 6 & 0.418685000 \\ 1 & 0.975771000 \\ 6 & 1.000764000 \\ 6 & 0.855555000 \\ 6 & 2.237432000 \\ 1 & 0.969586000 \\ 6 & -0.082420000 \\ 1 & -0.751220000 \\ 1 & 0.534048000 \\ 6 & -0.971194000 \\ 1 & -1.271339000 \\ 1 & -0.420079000 \\ 6 & -2.240752000 \\ 1 & -2.700157000 \\ 1 & -2.958524000 \\ 6 & -2.044809000 \\ 1 & -3.013238000 \\ 1 & -1.744392000\end{array}$

$$
\begin{array}{r}
-2.433109000 \\
-1.345151000 \\
-0.953778000 \\
-0.989761000 \\
-1.632469000 \\
-1.254844000 \\
0.442054000 \\
0.517403000 \\
0.506231000 \\
1.588689000 \\
1.809786000 \\
2.482595000 \\
1.416298000 \\
2.413339000 \\
0.976904000 \\
0.611444000 \\
0.998218000 \\
0.791933000 \\
-0.893417000 \\
-1.347591000 \\
-1.301315000
\end{array}
$$

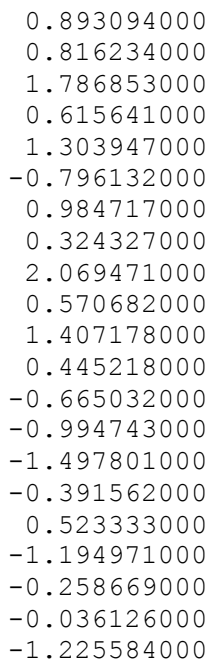




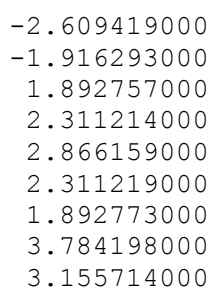

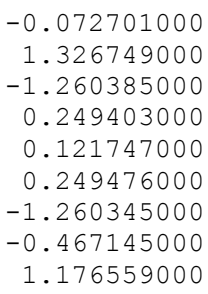

\section{Conformer 1c-II}

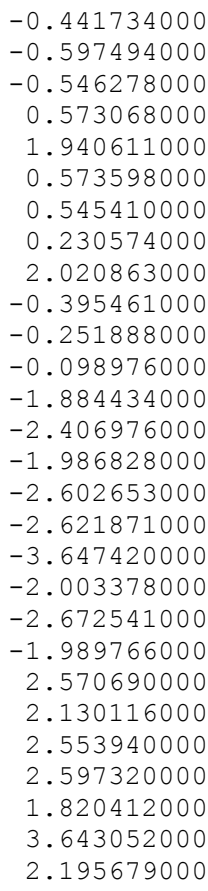

$-0.119392000$ $-0.214745000$ $-1.288650000$ 0.504253000 $-0.153660000$ 1. 527333000 0.604447000 1. 611098000 0.396417000 $-0.365579000$ $-1.393361000$ $-0.363423000$ 0.002891000 $-0.512318000$ 1.073274000 $-0.327472000$ $-1.415261000$ $-0.016708000$ 0.294753000 0.071675000 1. 386332000 1. 335146000 0.082733000 $-0.623786000$ 0.581214000 $-0.968572000$ $-0.671925000$ $-1.624315000$

\section{Conformer 1c-III}

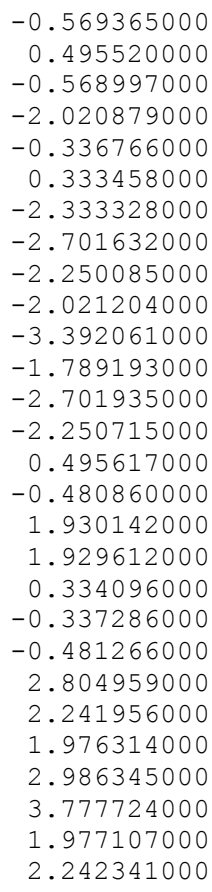

0.574213000 $-0.306589000$ 0.574748000 0.075740000 0.660216000 $-1.335567000$ $-0.669287000$ 0.924571000 $-0.600628000$ 0.075223000 $-0.941760000$ $-1.617262000$ 0.924058000 $-0.601399000$ $-0.306481000$ 1.588996000 0.100949000 0.101786000 $-1.335544000$ 0.658968000 1. 588746000 $-0.288096000$ $-0.357072000$ 1. 186113000 $-1.366767000$ 0.205150000 1. 185104000 $-0.359159000$ 


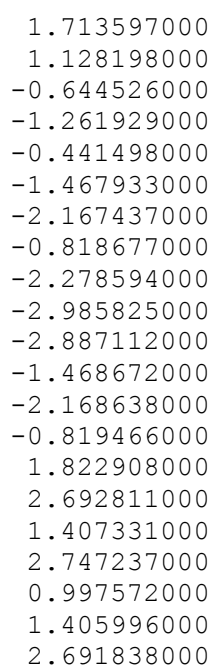

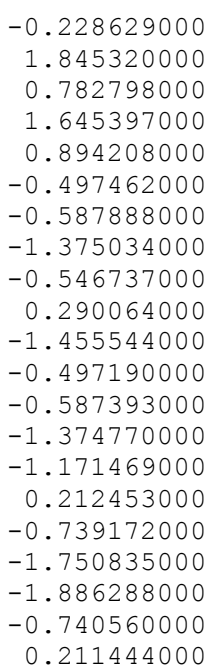

\section{Conformer 1c-VII}

\section{Conformer 1c-I}

2.565570000

1.497212000

1.206307000

0.785007000

1.189268000

1.107958000

$-0.785015000$

$-1.107975000$

$-1.189261000$

$-1.497220000$

$-1.206326000$

$-2.565580000$

$-1.294252000$

$-2.131058000$

$-1.339596000$

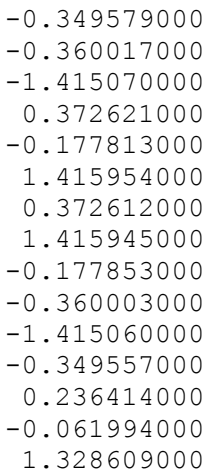

1.328609000 


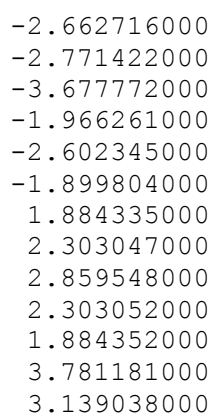

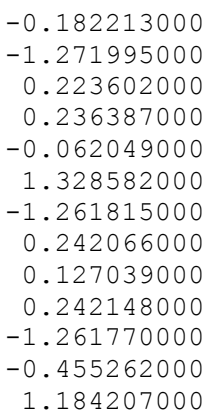

1.184207000

\section{Conformer 1c-II}

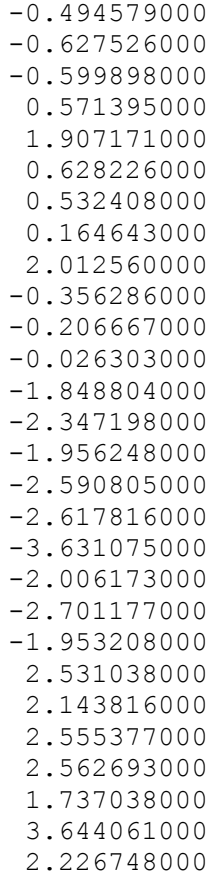

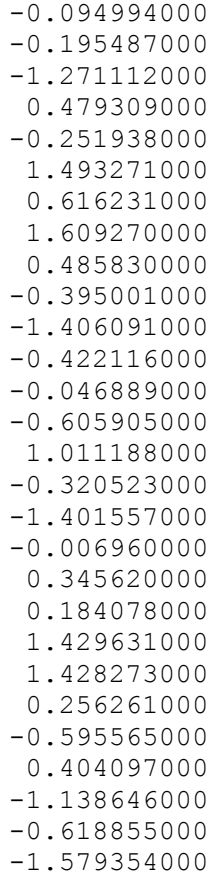

\section{Conformer 1c-III}

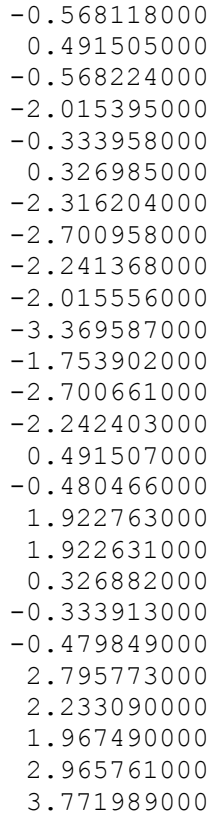

0.579043000

$-0.306013000$ 0.579307000 0.074856000 0.673371000 $-1.333320000$ $-0.677444000$ 0.920338000 $-0.597899000$ 0.075374000 $-0.967745000$ $-1.614088000$ 0.921321000 $-0.596802000$ $-0.306194000$ 1.588747000 0.102864000 0.102489000 $-1.333580000$ 0.672292000 1.588719000 $-0.293397000$ $-0.350534000$ 1.187597000 $-1.373588000$ 0.191848000 


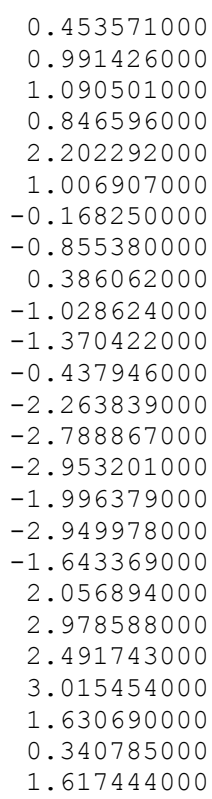

0.669060000 1.416354000 $-0.705952000$ 0.966987000 0.249800000 2. 042572000 0.589734000 1.429299000 0.511988000 $-0.668164000$ $-0.984189000$ $-1.499223000$ $-0.444357000$ 0.442297000 $-1.282177000$ $-0.281010000$ $-0.089516000$ $-1.232697000$ $-1.051539000$ 0.870495000 0.083885000 $-1.427114000$ $-1.832194000$ $-1.484927000$ $-0.641365000$

\section{Conformer 1c-VII}
0. 624522000
1.571884000
2. 342014000
1.687713000
2.721088000
1.489957000
0.769941000
0.769365000
$-0.607777000$
1. 686174000
2.719578000
1. 486264000
$-0.607370000$
$-1.502331000$
$-1.124124000$
$-1.124025000$
$-1.503154000$
0.627207000
1.287151000
$-2.420679000$
$-0.904036000$
$-2.052176000$
1. 286815000
0.627956000
$-2.053707000$
$-0.905253000$
$-2.884765000$
$-3.227063000$

$-1.680531000$

$-1.143889000$

$-1.919532000$

$-0.355280000$

$-0.026484000$

$-1.051842000$

0.874203000

0.874359000

0.811713000

$-0.355867000$

$-0.027999000$

$-1.052430000$

0.811162000

$-0.373413000$

1. 723364000

1. 724490000

$-0.371884000$

1.068575000

1. 754888000

$-0.606951000$

$-1.265978000$

$-0.165265000$

1. 755584000

1.067092000

$-0.162460000$

$-1.264359000$

$-1.593377000$

0.131340000

\section{d. B2PLYP/cc-pVTZ}

\section{Conformer 1c-I}

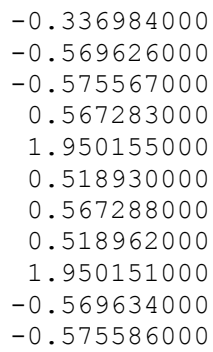

2.565252000

1. 497367000

1.208250000

0.784794000

1.187657000

1.109700000

$-0.784801000$

$-1.109716000$

$-1.187649000$

$-1.497375000$

$-1.208268000$
$-0.346865000$

$-0.359767000$

$-1.414850000$

0.372382000

$-0.180642000$

1. 415050000

0.372374000

1. 415041000

$-0.180681000$

$-0.359754000$

$-1.414840000$ 


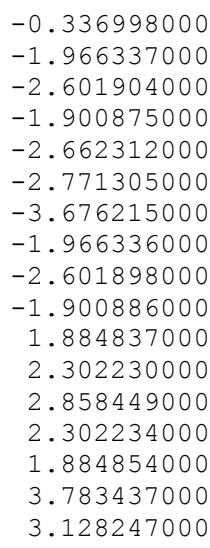

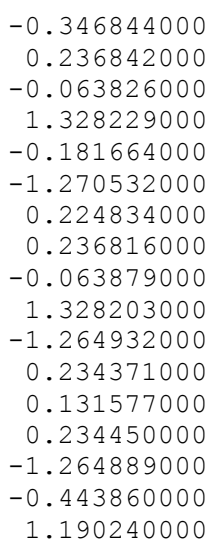

\section{Conformer 1c-II}

$-1.398735000$ $-0.399080000$ $-0.026546000$ $-0.568580000$ 1.035945000 $-0.324176000$ $-1.407065000$ $-0.012580000$ 0.325662000 0.140892000 1. 411988000 1. 389432000 0.201677000 $-0.616423000$ 0.481209000 $-1.063278000$ $-0.667306000$ $-1.599504000$

\section{Conformer 1c-III}

$$
\begin{array}{rr}
0.491223000 & -0.787833000 \\
-2.015318000 & -1.298496000 \\
-2.312813000 & 0.000004000 \\
-2.015316000 & 1.298495000 \\
-3.364576000 & 0.000006000 \\
-2.702278000 & -1.400170000 \\
-0.568628000 & 1.443573000 \\
-2.239781000 & 2.128986000 \\
-2.702268000 & 1.400153000 \\
0.491223000 & 0.787831000 \\
-0.334497000 & 2.506561000 \\
-0.481006000 & 1.038836000 \\
0.328504000 & 1.124485000 \\
1.922529000 & 1.188671000 \\
-0.568635000 & -1.443571000 \\
-1.747015000 & 0.000009000 \\
-2.239769000 & -2.128980000 \\
-0.334505000 & -2.506558000 \\
-0.481022000 & -1.038828000 \\
1.922526000 & -1.188670000 \\
0.328512000 & -1.124492000 \\
1.968182000 & 1.315566000
\end{array}
$$

-0.305564000
0.074442000
-0.678893000
0.074456000
-0.972525000
0.917880000
0.580424000
-0.598544000
0.917903000
-0.305569000
0.673022000
1.589674000
-1.332527000
0.105116000
0.580424000
-1.612700000
-0.598572000
0.673030000
1.589672000
0.105131000
-1.332523000
1.190474000 
2.232293000
2.793596000
3.772414000
2.954018000
2.232290000
1.968175000

2.132370000 $-0.000004000$ $-0.000002000$ $-0.000011000$ $-2.132377000$ $-1.315549000$
-0.343131000
-0.298240000
0.180024000
-1.378910000
-0.343101000
1.190491000

\section{Conformer 1c-IV}

$-0.348155000$

$-1.351405000$ 0.559682000

1. 563353000 0.024441000 0.643461000 1. 569630000 $-0.495292000$ $-0.356976000$ $-0.418072000$ $-1.464315000$ 0.446329000 $-1.257528000$ $-0.850114000$

1.660732000

\section{Conformer 1c-V}

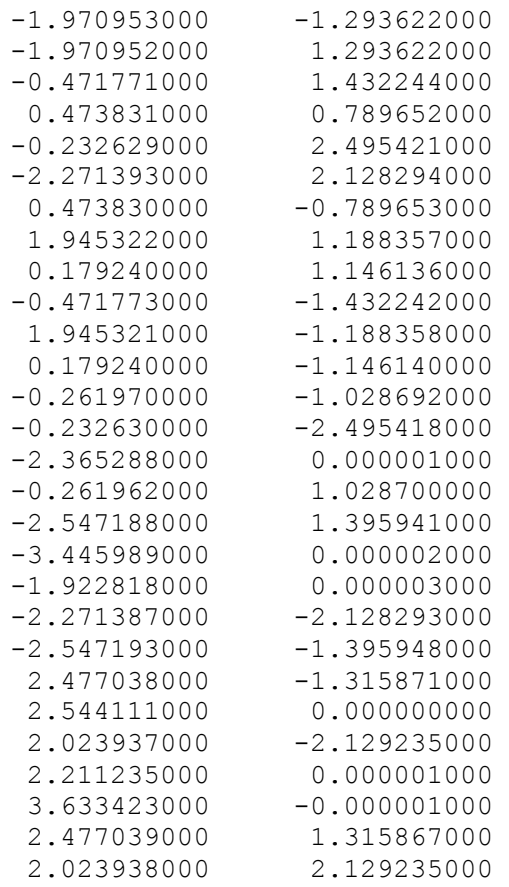

$-0.190458000$ $-0.190463000$ $-0.513630000$ 0.509200000 $-0.575645000$ 0.446796000 0.509198000 0.239634000 1. 497970000 $-0.513632000$ 0.239631000 1. 497967000 $-1.504756000$ $-0.575654000$ 0.522111000 $-1.504754000$ $-1.113020000$ 0.678510000 1. 520093000 0.446807000 $-1.113012000$

1. 184721000 $-0.510649000$ $-0.304479000$ $-1.550726000$ $-0.516716000$ 1. 184724000 $-0.304474000$ 


\section{Conformer 1c-VII}

$-0.019089000$

$-2.124033000$

$-1.049517000$

$-1.453950000$

0.873342000

1.454266000

0.873117000

0.806762000

1.307192000

1.446494000

$-0.352401000$

2.124192000

$-0.019481000$

$-1.049973000$

$-0.786743000$

$-1.204146000$

$-1.094061000$

0.806725000

$-0.377284000$

1.718192000

1.093687000

1.718362000

1.203856000

2.520340000

1.071313000

$-0.000387000$

$-1.383109000$

$-2.124371000$

$-1.070682000$

$-2.520043000$

2.123686000

1.383484000

$-0.000397000$

$-0.000718000$

$-0.376971000$

1.064942000

1.756355000

$-0.599409000$

$-1.273119000$

$-0.170858000$

1.756563000

1.065322000

$-0.170175000$

$-1.272830000$

$-1.578641000$

0.150736000

\section{e. B2PLYP-D3/cc-pVTZ}

\section{Conformer 1c-I}




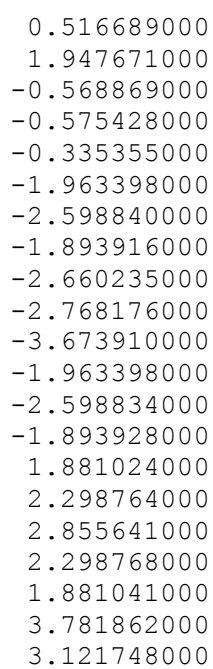

1. 414783000 $-0.180916000$ $-0.361931000$ $-1.414883000$ $-0.355151000$ 0.237828000 $-0.059434000$ 1. 328923000 $-0.180747000$ $-1.269568000$ 0.225737000 0.237801000 $-0.059490000$ 1. 328896000 $-1.265353000$ 0.231532000 0.133490000 0.231614000 $-1.265307000$ $-0.439582000$ 1.192954000

\section{Conformer 1c-II}

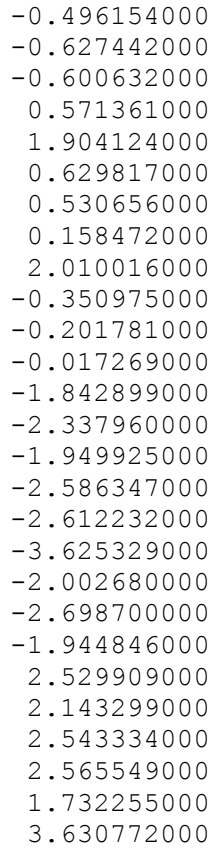

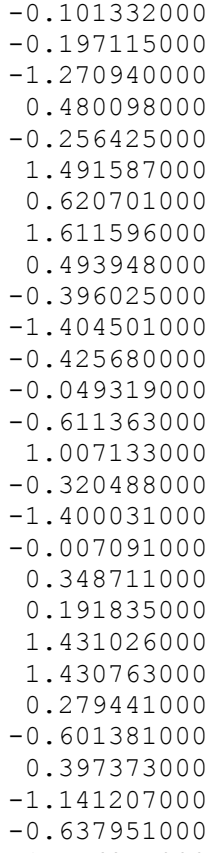

\section{Conformer 1c-III}

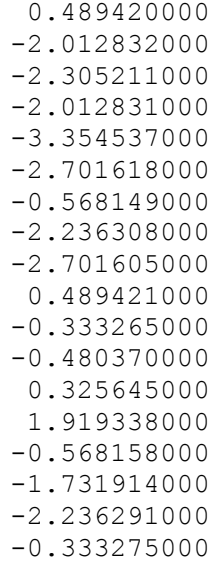

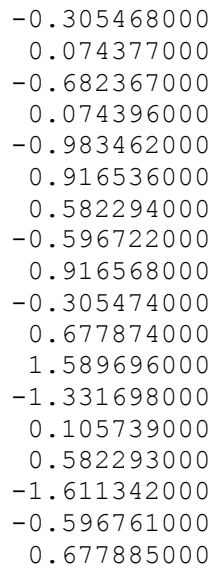

0.677885000 


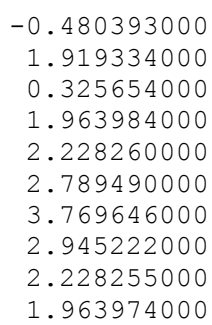

1.589693000 0.105759000 $-1.331692000$ 1.191438000 $-0.339604000$ $-0.300405000$ 0.174472000 $-1.381641000$ $-0.339563000$ 1.191461000

\section{Conformer 1c-IV}

\section{Conformer 1c-V}

$-1.291613000$

1. 291612000

1. 429119000

0.789019000

2. 491554000

2.127743000

$-0.789020000$

1.186880000

1.146453000

$-1.429117000$

$-1.186881000$

$-1.146457000$

$-1.021677000$

$-2.491551000$

0.000001000

1.021686000

1. 389902000

0.000002000

0.000003000

$-2.127742000$

$-1.389910000$

$-1.308794000$

0.000000000

$-2.129459000$

0.000001000

$-0.000001000$

1. 308790000

2. 129458000

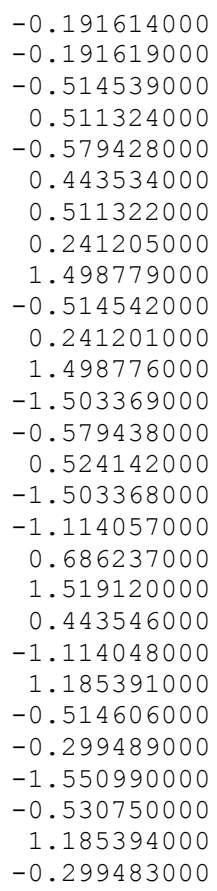




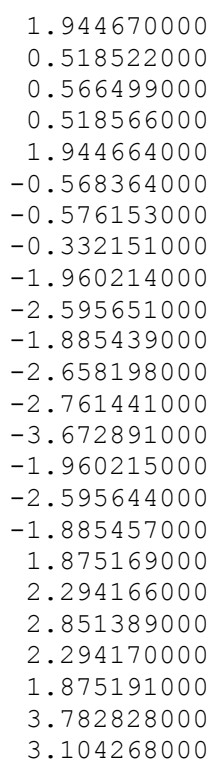

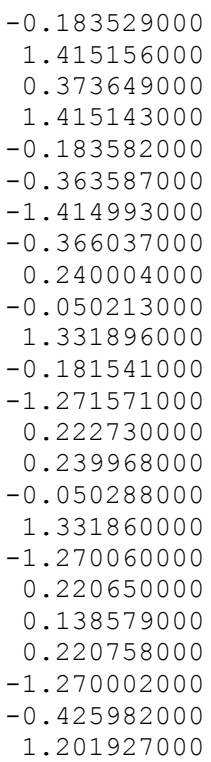

\section{Conformer 1c-II}

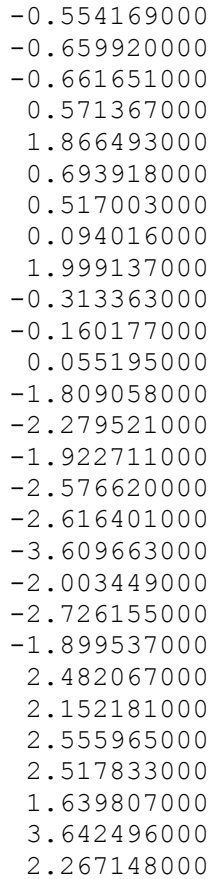

2.642264000

1. 560746000

1. 373258000

0.907987000

1. 188169000

1.335343000

$-0.629884000$

$-0.875696000$

$-1.019644000$

$-1.372452000$

$-0.937857000$

$-2.399598000$

$-1.422434000$

$-2.196568000$

$-1.742085000$

$-0.119781000$

0.100736000

$-0.273431000$

1.104946000

1. 917835000

0.901467000

$-0.728016000$

$-2.090761000$

$-0.172679000$

1.868901000

1. 668875000

$-0.102180000$

$-0.612325000$

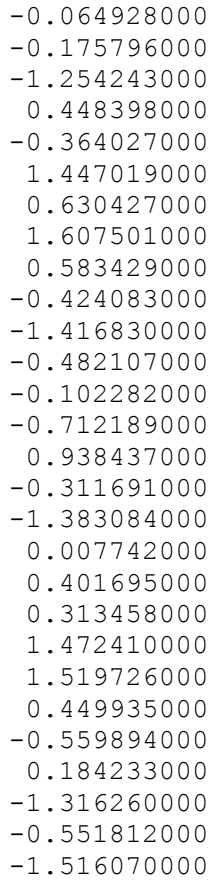

\section{Conformer 1c-III}

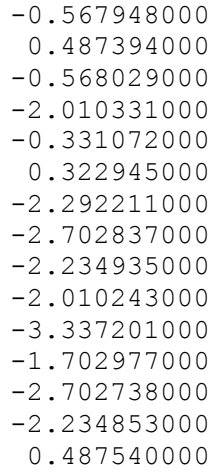

0.584474000 $-0.303952000$ 0.584453000 0.074446000 0.678282000 $-1.330436000$ $-0.688591000$ 0.915086000 $-0.593768000$ 0.074484000 $-1.004846000$ $-1.609011000$ 0.915139000 $-0.593677000$ $-0.303855000$ 


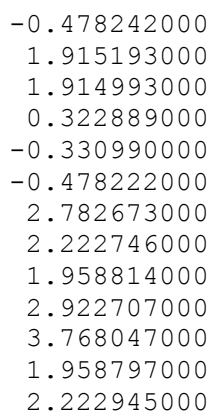

1.593206000 0.108364000 0.108258000 $-1.330341000$ 0.678205000 1. 593276000 $-0.306786000$ $-0.329149000$ 1.196318000 $-1.390814000$ 0.157325000 1.196436000 $-0.328995000$

\section{Conformer 1c-IV}

\section{Conformer $\mathbf{1 c}-\mathbf{V}$}




\section{3. trans-Perhydroazulene}

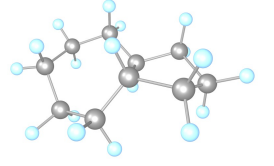

1t-I

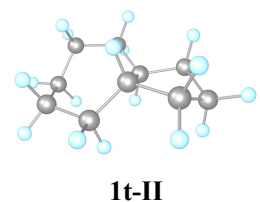

1t-II

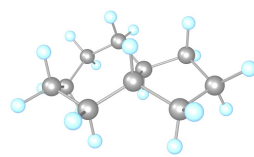

1t-III

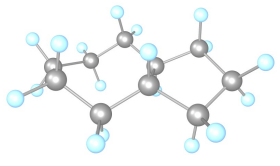

1t-IV

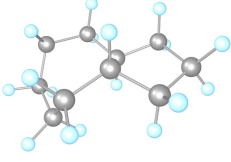

1t-V

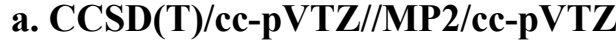

\section{Conformer 1t-I}

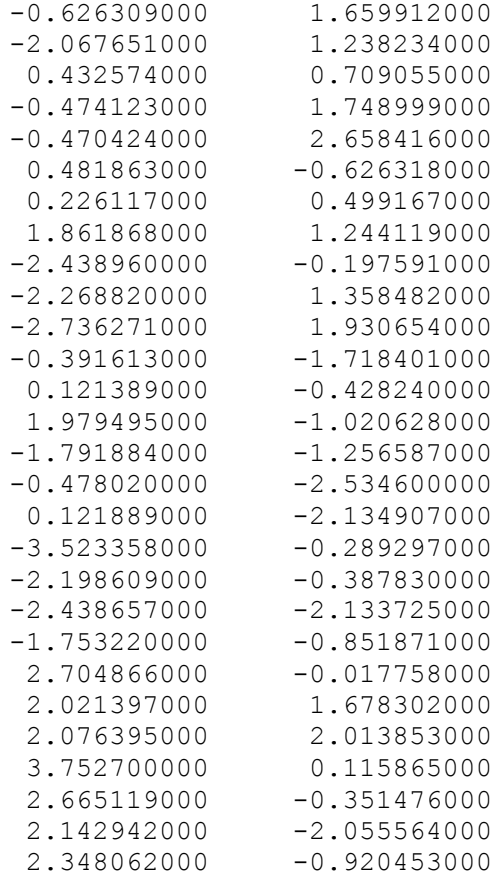

0.241824000 $-0.038282000$ $-0.298563000$ 1. 322441000 $-0.175792000$ 0.474818000

$-1.355266000$ $-0.203449000$ 0.344502000

$-1.106970000$ 0.477969000 $-0.134241000$ 1. 487285000 0.548319000

$-0.556629000$

0.587548000

$-1.005802000$

0.260740000

1.393990000

$-0.611592000$

$-1.570722000$

$-0.357510000$

0.788554000

$-0.945009000$

$-0.092130000$

$-1.396700000$

0.246972000

1. 569746000

\section{$\boldsymbol{E}[\mathrm{MP} 2]=-391.0187458$ a.u. \\ $\boldsymbol{Z P V E}[\mathrm{MP} 2]=166.70418 \mathrm{kcal} \mathrm{mol}^{-1}$ \\ $\boldsymbol{E}[\mathrm{CCSD}(\mathrm{T})]=-391.1839846$ a.u.}

\section{Conformer 1t-II}

$$
\begin{array}{r}
1.947290000 \\
0.463279000 \\
0.123431000 \\
0.460126000 \\
1.907947000 \\
0.252459000 \\
-0.567460000 \\
-0.635433000 \\
-0.203679000 \\
-1.964386000 \\
-2.012668000 \\
-2.686276000 \\
-2.400458000 \\
-3.490982000 \\
-2.056110000 \\
-1.947836000 \\
-2.262734000 \\
-2.487604000 \\
-0.453833000 \\
-0.274654000 \\
-0.164611000
\end{array}
$$

$-0.626096000$

$-0.453307000$

0.712311000

1. 198075000

0.472076000

1. 731598000

2. 524770000

2.205392000

1. 155001000

0.758540000

1. 972510000

0.040345000

0.031079000

0.250625000

$-1.353811000$

$-1.486977000$

$-2.105570000$

$-1.655969000$

$-2.636684000$

$-1.741177000$

0.510731000

0.490012000

1. 515958000

$-0.280363000$

$-0.215583000$

$-1.329071000$

0.191288000

$-0.557592000$

1. 107293000

0.450079000

1. 467023000

0.413948000

$-0.507499000$

$-0.553096000$

$-1.523687000$

$-0.063806000$

0.975599000

$-0.644062000$

$-0.163925000$

0.284557000

$-1.216873000$

$-0.392804000$ 


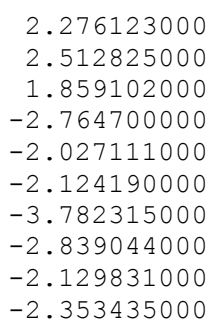

1.407198000 $-0.494167000$ $-1.549858000$ $-0.309240000$ 0.834143000 $-0.889415000$ 0.057807000 $-1.365803000$ 0.024590000 1.491026000

\section{Conformer 1t-II}

\section{Conformer 1t-III}




\section{Conformer 1t-IV}
0.350392000
0.000000000
$-1.085309000$
0.670796000
0.758543000
1. 699053000
0.000000000
0.758526000
$-0.758526000$
$-0.670796000$
$-1.699053000$
$-0.758543000$
0.000000000
$-0.350392000$
1.085309000
$-0.328229000$
$-1.415160000$
0.048269000
0.328229000
$-0.048269000$
1. 415160000
0.000000000
1.059309000
$-0.612356000$
0.872890000
$-0.872890000$
$-1.059309000$
0.612356000

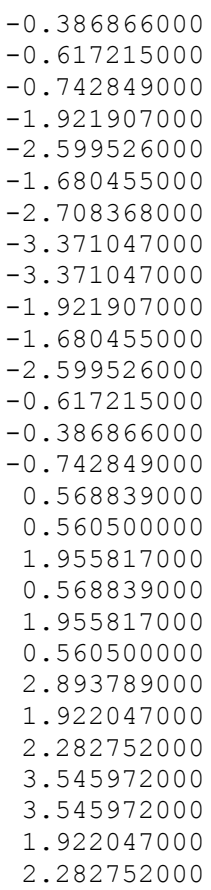

\section{Conformer 1t-V}

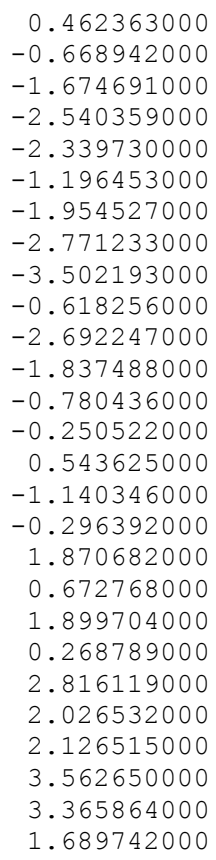

0.287482000

$-0.481444000$

0.636172000

0.407420000

0.751815000

$-1.437817000$

$-0.535285000$

1. 373784000

$-0.011930000$

$-0.115067000$

$-0.657782000$

$-1.527777000$

0.714208000

$-0.948266000$

$-0.562987000$

1. 584569000

$-0.340776000$

$-0.086815000$

$-1.606963000$

0.237931000

1. 318408000

0.184392000

$-0.666872000$

1. 081617000

$-0.603633000$

1. 115615000

0.837177000

$-0.806171000$

\section{c. B3LYP-D3(BJ)/cc-pVTZ}

\section{Conformer 1t-I}

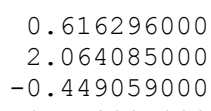




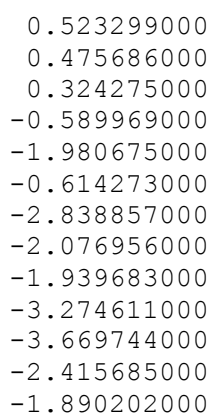

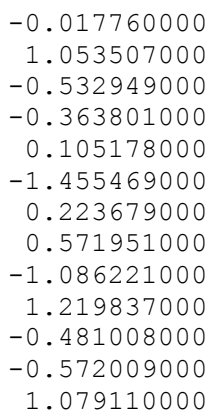

\section{Conformer 1t-IV}

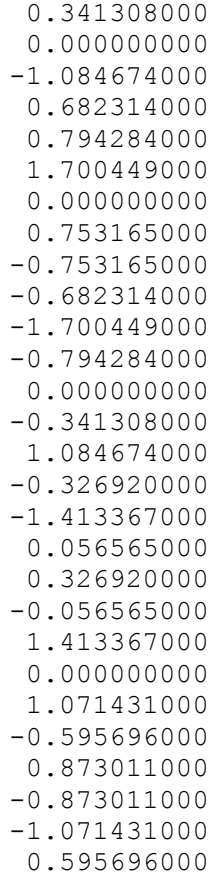

2.605822000

1.593024000

1. 660517000

1.130684000

1. 981094000

0.825967000

0.000000000

$-0.436394000$

0.436394000

$-1.130684000$

$-0.825967000$

$-1.981094000$

$-1.593024000$

$-2.605822000$

$-1.660517000$

$-0.692848000$

$-0.532732000$

$-1.233160000$

0.692848000

1. 233160000

0.532732000

0.000000000

$-1.636820000$

$-2.043283000$

0.037125000

$-0.037125000$

1. 636820000

2.043283000

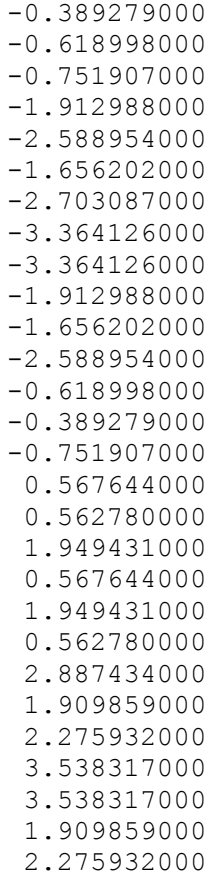

\section{Conformer 1t-V}

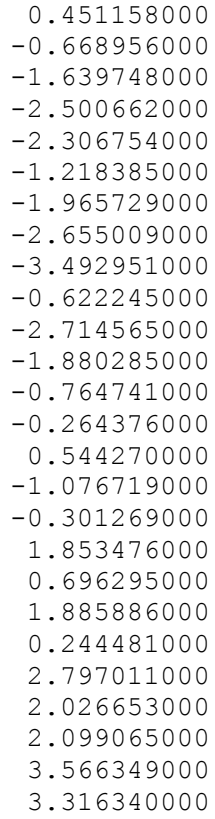

0.267008000

$-0.523151000$

0.629469000

0.462736000

0.736187000

$-1.465524000$

$-0.516394000$

0.111958000

$-0.147438000$

$-0.619798000$

$-1.505972000$

0.663174000

$-1.006029000$

$-0.583323000$

1. 562803000

$-0.435477000$

$-0.075472000$

$-1.623136000$

0.234370000

1. 294167000

0.215117000

$-0.678657000$

1. 071052000

$-0.551104000$

1. 163655000
1. 441579000 


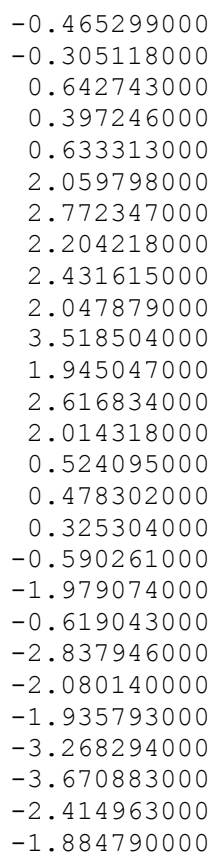

0.201831000 1.285082000 $-0.441470000$ $-0.351492000$ $-1.513755000$ 0.116398000 $-0.639587000$ 0.974647000 0.554127000 1.560740000 0.642039000 $-0.349677000$ $-0.227699000$ $-1.401712000$ $-0.021098000$ 1.048943000 $-0.539544000$ $-0.363331000$ 0.110918000 $-1.454566000$ 0.225324000 0.552713000 $-1.098401000$ 1.222355000 $-0.475554000$ $-0.561565000$ 1.086552000

\section{Conformer 1t-IV}
0.343906000
0.000000000
$-1.084006000$
0.683069000
0.795183000
1. 700283000
0.000000000
0.752284000
$-0.752284000$
$-0.683069000$
$-1.700283000$
$-0.795183000$
0.000000000
$-0.343906000$
1. 084006000
$-0.325609000$
$-1.412307000$
0.066012000
0.325609000
$-0.066012000$
1. 412307000
0.000000000
1.084280000
$-0.577530000$
0.872333000
$-0.872333000$
$-1.084280000$
0.577530000

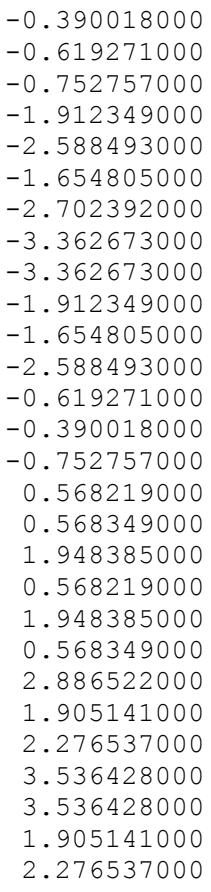

\section{Conformer 1t-V}

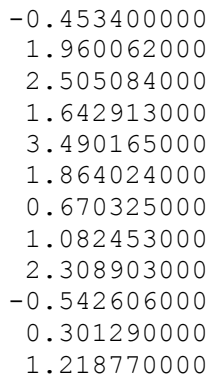

0.687828000 


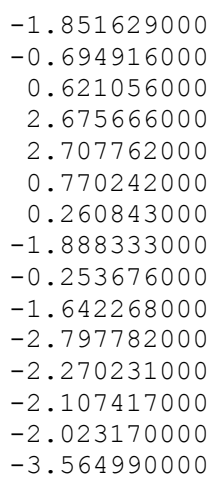

0.074465000 1.620456000 0.136845000 $-1.428566000$ 0.629441000 $-0.679266000$ 0.988096000 $-0.228858000$ $-1.300120000$ $-0.848111000$ $-0.211399000$ 0.779909000 $-1.059907000$ 0.688579000 0.556072000 $-1.158923000$

\section{e. B2PLYP-D3/cc-pVTZ}

\section{Conformer 1t-I}

$$
\begin{array}{r}
-0.618551000 \\
-2.064373000 \\
0.444958000 \\
-0.473050000 \\
-0.456206000 \\
0.487474000 \\
0.246954000 \\
1.875315000 \\
-2.459419000 \\
-2.259977000 \\
-2.726585000 \\
-0.414166000 \\
0.150327000 \\
1.983901000 \\
-1.823375000 \\
-0.492491000 \\
0.077197000 \\
-3.544158000 \\
-2.231248000 \\
-2.473123000 \\
-1.802370000 \\
2.732545000 \\
2.023786000 \\
2.096568000 \\
3.766378000 \\
2.747292000 \\
2.136802000 \\
2.348054000
\end{array}
$$

1. 661863000

1. 252315000

0.709754000

1.752625000

2.659953000

$-0.637534000$

0.512212000

1. 245653000

$-0.176208000$

1. 369250000

1.956950000

$-1.720703000$

$-0.441185000$

$-1.052085000$

$-1.259509000$

$-2.533238000$

$-2.148882000$

$-0.253826000$

$-0.357417000$

$-2.135557000$

$-0.882771000$

$-0.009824000$

1.684657000

2.016697000

0.127628000

$-0.316259000$

$-2.067796000$

$-1.025365000$
0.238004000 $-0.047765000$ $-0.299034000$ 1.318951000 $-0.176788000$ 0.459753000 $-1.358672000$ $-0.186913000$ 0.346076000 $-1.117620000$ 0.459560000 $-0.132020000$ 1. 479639000 0.511656000 $-0.536537000$ 0.594171000 $-1.009795000$ 0.256019000 1. 399572000 $-0.558367000$ $-1.561529000$ $-0.334472000$ 0.804124000 $-0.924619000$ $-0.021327000$ $-1.382290000$ 0.147863000 1.538629000

\section{Conformer 1t-II}

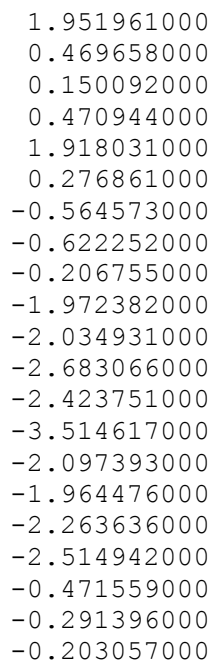




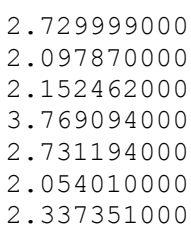

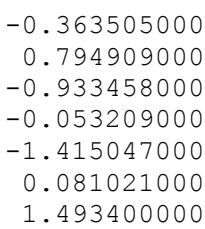

\section{Conformer 1t-III}

\section{Conformer 1t-IV}




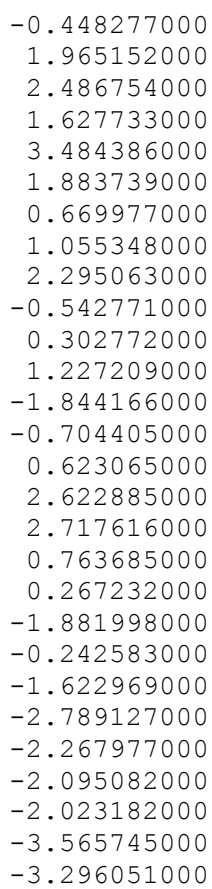

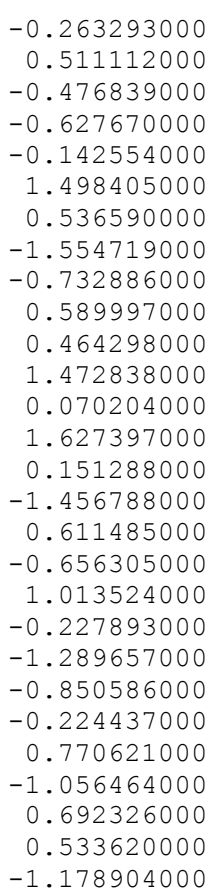

\section{f. M06-2X/cc-pVTZ}

\section{Conformer 1t-I}

\section{Conformer 1t-II}




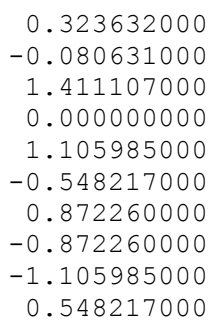

0.569489000 1.942770000 0.578591000 2.880067000 1.889747000 2.271534000 3.529481000 3.529481000 1.889747000 2.271534000

\section{Conformer 1t-V}

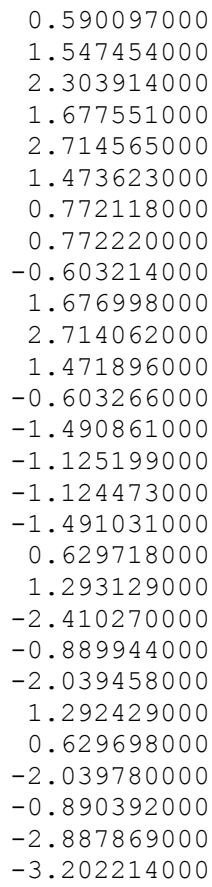

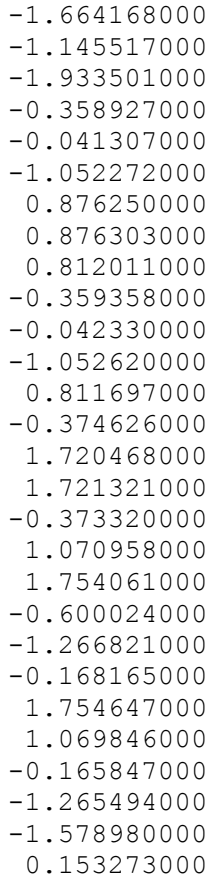


Table S11. Absolute energies of B3LYP/cc-pVTZ optimized structures of 1c and 1t (in a.u.)

\begin{tabular}{|c|c|c|c|}
\hline & $H_{0}$ & $G_{298}$ & $G_{550}$ \\
\hline 1c-I & 391.790380 & 391.824551 & 391.868992 \\
\hline 1c-II & 391.787202 & 391.822115 & 391.867231 \\
\hline 1c-III & 391.785822 & 391.819775 & 391.864010 \\
\hline $1 \mathrm{c}-\mathrm{IV}$ & 391.783845 & 391.818079 & 391.862695 \\
\hline $1 c-V$ & 391.782466 & 391.816934 & 391.861659 \\
\hline $1 \mathrm{c}-\mathrm{VI}$ & 391.782997 & 391.816408 & 391.860019 \\
\hline 1c-VII & 391.779013 & 391.812520 & 391.856328 \\
\hline 1t-I & 391.788729 & 391.822833 & 391.867324 \\
\hline 1t-II & 391.788641 & 391.822935 & 391.867637 \\
\hline 1t-III & 391.788754 & 391.823688 & 391.869099 \\
\hline 1t-IV & 391.787460 & 391.821488 & 391.866125 \\
\hline $1 t-V$ & 391.782050 & 391.817150 & 391.862652 \\
\hline
\end{tabular}

Table S12. Absolute energies of B3LYP-D3(BJ)/cc-pVTZ optimized structures of 1c and 1t (in a.u.)

\begin{tabular}{|c|c|c|c|}
\hline & $H_{0}$ & $G_{298}$ & $G_{550}$ \\
\hline 1c-I & 391.839289 & 391.873530 & 391.917981 \\
$\mathbf{1 c - I I}$ & 391.836578 & 391.871024 & 391.915660 \\
$\mathbf{1 c - I I I}$ & 391.835485 & 391.869403 & 391.913541 \\
$\mathbf{1 c - I V}$ & 391.833658 & 391.867841 & 391.912349 \\
$\mathbf{1 c - V}$ & 391.832577 & 391.866962 & 391.911539 \\
$\mathbf{1 c - V I}$ & 391.831642 & 391.866149 & 391.910879 \\
$\mathbf{1 c}-V I I$ & 391.830154 & 391.863508 & 391.907085 \\
\hline $\mathbf{1 t}-\mathbf{I}$ & 391.837844 & 391.871769 & 391.916013 \\
$\mathbf{1 t}-\mathbf{I I}$ & 391.837684 & 391.871765 & 391.916185 \\
$\mathbf{1 t}-\mathbf{I I I}$ & 391.837667 & 391.872553 & 391.917860 \\
$\mathbf{1 t}-I V$ & 391.836259 & 391.870173 & 391.914648 \\
$\mathbf{1 t}$ & 391.831640 & 391.866508 & 391.911729 \\
\hline
\end{tabular}


Table S13. Absolute energies of B2PLYP/cc-pVTZ optimized structures of 1c and 1t (in a.u.)

\begin{tabular}{|c|c|c|c|}
\hline & $H_{0}$ & $G_{298}$ & $G_{550}$ \\
\hline 1c-I & 391.401650 & 391.435845 & 391.480223 \\
1c-II & 391.398686 & 391.433351 & 391.478153 \\
1c-III & 391.397733 & 391.431638 & 391.475729 \\
1c-IV & 391.395533 & 391.429623 & 391.473999 \\
1c-V & 391.394565 & 391.428934 & 391.473466 \\
1c-VI & 391.392629 & 391.426847 & 391.471301 \\
1c-VII & 391.391107 & 391.424491 & 391.468074 \\
\hline 1t-I & 391.400138 & 391.434065 & 391.478276 \\
1t-II & 391.399957 & 391.434049 & 391.478447 \\
1t-III & 391.400005 & 391.434881 & 391.480147 \\
1t-IV & 391.398618 & 391.432504 & 391.476919 \\
1t-V & 391.393744 & 391.428652 & 391.473878 \\
\hline
\end{tabular}

Table S14. Absolute energies of B2PLYP-D3/cc-pVTZ optimized structures of 1c and 1t (in a.u.)

\begin{tabular}{|c|c|c|c|}
\hline & $H_{0}$ & $G_{298}$ & $G_{550}$ \\
\hline 1c-I & 391.424026 & 391.458262 & 391.502654 \\
1c-II & 391.421327 & 391.455793 & 391.500389 \\
1c-III & 391.420465 & 391.454364 & 391.498425 \\
1c-IV & 391.418365 & 391.452430 & 391.496756 \\
1c-V & 391.417538 & 391.451876 & 391.496351 \\
1c-VI & 391.415869 & 391.449875 & 391.494098 \\
1c-VII & 391.414619 & 391.447937 & 391.491419 \\
\hline 1t-I & 391.422630 & 391.456489 & 391.500604 \\
1t-II & 391.422415 & 391.456422 & 391.500706 \\
1t-III & 391.422378 & 391.457227 & 391.502442 \\
1t-IV & 391.420937 & 391.454778 & 391.499126 \\
1t-V & 391.416472 & 391.451324 & 391.496471 \\
\hline
\end{tabular}


Table S15. Absolute energies of M06-2X/cc-pVTZ optimized structures of 1c and 1t (in a.u.)

\begin{tabular}{|c|c|c|c|}
\hline & $H_{0}$ & $G_{298}$ & $G_{550}$ \\
\hline 1c-I & 391.594951 & 391.629834 & 391.674771 \\
$\mathbf{1 c - I I}$ & 391.592479 & 391.626878 & 391.671401 \\
$\mathbf{1 c - I I I}$ & 391.591820 & 391.625771 & 391.669841 \\
$\mathbf{1 c - I V}$ & 391.589664 & 391.623488 & 391.667521 \\
$\mathbf{1 c - V}$ & 391.588936 & 391.623121 & 391.667406 \\
$\mathbf{1 c - V I}$ & 391.586626 & 391.620369 & 391.664313 \\
$\mathbf{1 c}-V I I$ & 391.585935 & 391.619180 & 391.662568 \\
\hline $\mathbf{1 t}-\mathbf{I}$ & 391.593753 & 391.627446 & 391.671351 \\
$\mathbf{1 t - I I}$ & 391.593423 & 391.627232 & 391.671269 \\
$\mathbf{1 t}-\mathbf{I I I}$ & 391.593632 & 391.628564 & 391.673836 \\
$\mathbf{1 t - I V}$ & 391.592009 & 391.625729 & 391.669946 \\
$\mathbf{1 t}$ & 391.587893 & 391.622427 & 391.667232 \\
\hline
\end{tabular}

Table S16. Absolute energies of MP2/cc-pVTZ optimized structures of 1c and 1t (in a.u.)

\begin{tabular}{|c|c|c|c|}
\hline & $H_{0}$ & $G_{298}$ & $G_{550}$ \\
\hline 1c-I & 390.754145 & 390.788512 & 390.833048 \\
1c-II & 390.751799 & 390.786159 & 390.830687 \\
1c-III & 390.751460 & 390.785360 & 390.829431 \\
1c-IV & 390.748936 & 390.782852 & 390.827038 \\
1c-V & 390.748719 & 390.782950 & 390.827328 \\
1c-VI & 390.745885 & 390.779659 & 390.823671 \\
1c-VII & 390.745239 & 390.778511 & 390.821966 \\
\hline 1t-I & 390.753086 & 390.786818 & 390.830806 \\
1t-II & 390.752754 & 390.786634 & 390.830793 \\
1t-III & 390.752572 & 390.787375 & 390.832560 \\
1t-IV & 390.751063 & 390.784792 & 390.829047 \\
1t-V & 390.747299 & 390.782027 & 390.827070 \\
\hline
\end{tabular}




\section{1,2-Dimethylcycloheptane}

Geometries were optimized at MP2/cc-pVTZ and single point energies were computed at CCSD(T)/ cc-pVTZ. For geometry optimizations, we only considered the $\mathbf{T C}$ and $\mathbf{C}$ conformations as starting conformations. We found only the TC conformation in the geometry optimized structures for both the cis- and trans-isomers.

\section{a. trans-1,2-Dimethylcycloheptane}

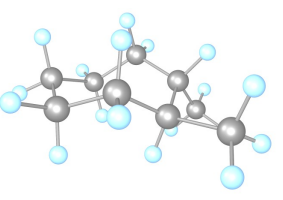

conformer 1

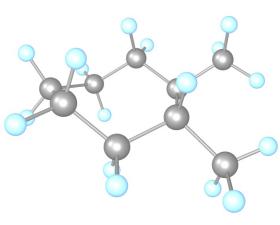

conformer 2

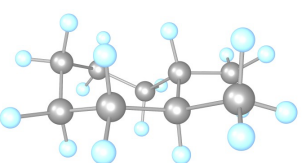

conformer 3

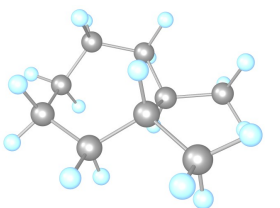

conformer 4

\section{Conformer 1}

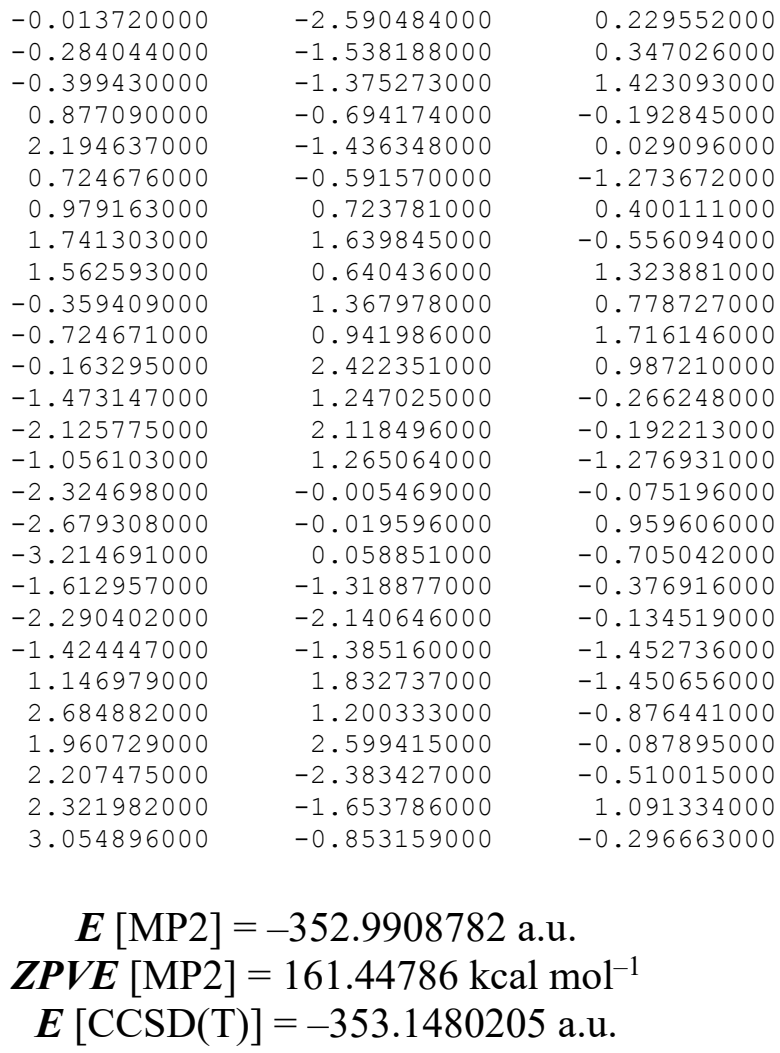

\section{Conformer 2}

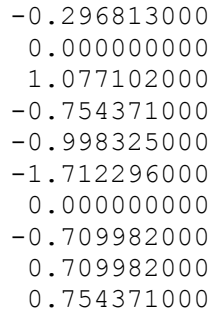

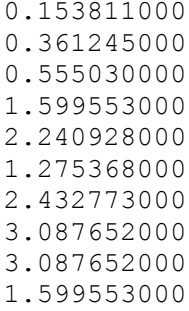



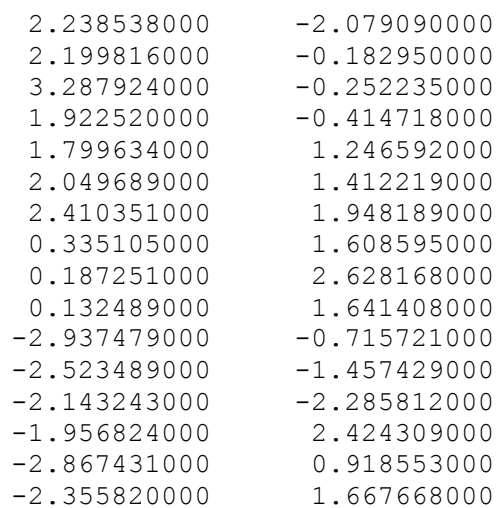

0.631933000 $-0.401568000$ $-0.352327000$ $-1.433916000$ $-0.047979000$ 1.004127000 $-0.620805000$ $-0.272482000$ 0.092984000 $-1.348343000$ $-0.856625000$ 0.691266000 $-0.813891000$ 0.830808000 0.804154000 $-2.355820000$

1.667668000

$-0.708114000$

$$
\begin{aligned}
& \boldsymbol{E}[\mathrm{MP} 2]=-352.9884664 \text { a.u. } \\
& \boldsymbol{Z P V E}[\mathrm{MP} 2]=161.61521 \mathrm{kcal} \mathrm{mol}^{-1} \\
& \boldsymbol{E}[\mathrm{CCSD}(\mathrm{T})]=-353.1457069 \text { a.u. }
\end{aligned}
$$

\section{b. cis-1,2-Dimethylcycloheptane}

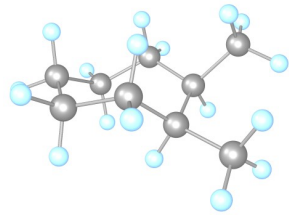

$$
\begin{array}{r}
0.270321000 \\
-0.098734000 \\
0.003976000 \\
0.771761000 \\
2.132493000 \\
0.263019000 \\
0.919021000 \\
1.267452000 \\
1.954455000 \\
-0.399899000 \\
-0.550942000 \\
-0.274321000 \\
-1.665306000 \\
-2.386033000 \\
-1.451212000 \\
-2.327234000 \\
-2.464900000 \\
-3.327868000 \\
-1.571202000 \\
-2.091846000 \\
-1.620065000 \\
1.717973000 \\
1.953641000 \\
2.963725000 \\
2.011153000 \\
2.648985000 \\
2.774813000
\end{array}
$$

$\boldsymbol{E}[\mathrm{MP} 2]=-352.9899782$ a.u.

$\boldsymbol{Z P V E}[\mathrm{MP} 2]=161.60172 \mathrm{kcal} \mathrm{mol}^{-1}$

$\boldsymbol{E}[\mathrm{CCSD}(\mathrm{T})]=-353.1470827$ a.u. 


\section{References}

[1] Krause, L.; Herbst-Irmer, R.; Sheldrick, G. M.; Stalke, D. J. Appl. Cryst. 2015, 48, 3-10.

[2] Sheldrick, G. M. Acta Cryst. 2015, A71, 3-8.

[3] Sheldrick, G. M. Acta Cryst. 2015, C71, 3-8.

[4] Crystallographic data can be obtained free of charge at https://ccdc.cam.ac.uk/structures/

[5] Müller, P. Cryst. Rev. 2009, 15, 57-83.

[6] Thorn, A.; Dittrich, B.; Sheldrick, G. M. Acta Cryst. 2012, A68, 448-451. 